\title{
Structures and Self-Organization at Liquid Crystal Interfaces: Surface Ordering and Anchoring
}

\section{Dissertation}

zur Erlangung des mathematisch-naturwissenschaftlichen Doktorgrades

"Doctor rerum naturalium" der Georg-August-Universität Göttingen

im Promotionsprogramm ProPhys

der Georg-August University School of Science (GAUSS)

vorgelegt von

\section{Xunda Feng}

aus Guangxi, China

Göttingen, 2013 



\section{Betreuungsausschuss:}

Prof. Dr. Christoph Schmidt, Third Institute of Physics

Prof. Dr. Stephan Herminghaus, Max Planck Institute for Dynamics and Self-Organization

Dr. Christian Bahr, Max Planck Institute for Dynamics and Self-Organization

\section{Mitglieder der Prüfungskommission:}

Referent: Prof. Dr. Christoph Schmidt, Third Institute of Physics

Korreferent: Prof. Dr. Stephan Herminghaus, Max Planck Institute for Dynamics and SelfOrganization

\section{Weitere Mitglieder der Prüfungskommission:}

Dr. Christian Bahr, Max Planck Institute for Dynamics and Self-Organization

Dr. Jean-Christophe Baret, Max Planck Institute for Dynamics and Self-Organization

Prof. Dr. Sarah Köster, Institute of X-Ray Physics

Prof. Marcus Müller, Intistute of Theoretical Physics

Tag der mündlichen Prüfung: 15-04-2013 



\section{Contents}

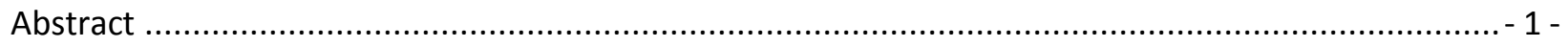

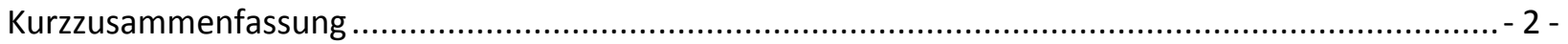

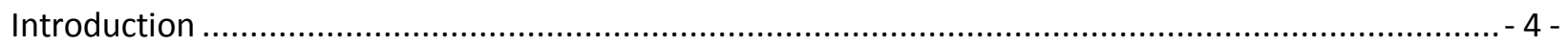

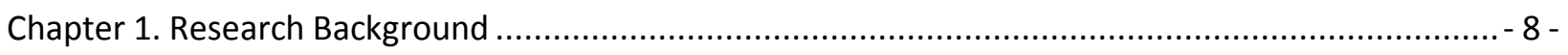

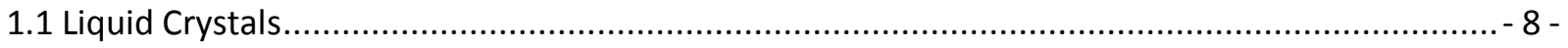

1.1.1 Liquid Crystal: the Forth State of Matter .................................................................... 8 -

1.1.2 Thermotropic Liquid Crystalline Phases ....................................................................... 10 -

1.1.3 Structure and Properties of Thermotropic Liquid Crystals........................................... 13 -

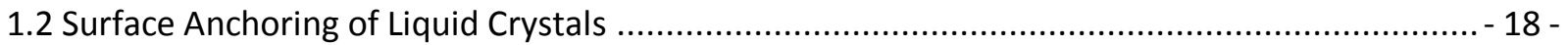

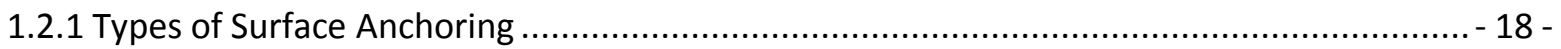

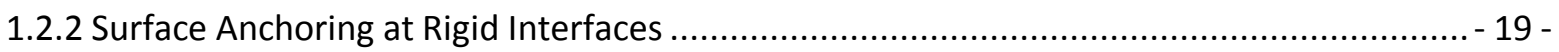

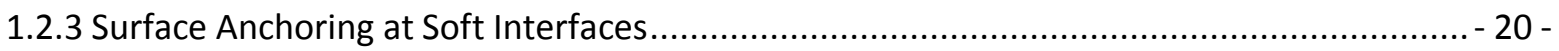

1.3 Surface Ordering at Surfaces and Interfaces of Isotropic Liquids.......................................... 23 -

1.4 Surface-Induced Pretransitional Ordering of Liquid Crystals ................................................ 27 -

Chapter 2. Experimental Techniques and Theoretical Models ................................................... 32 -

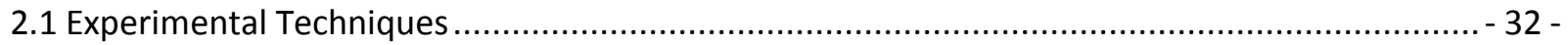

2.1.1 Basic Optics for Liquid Crystal Research................................................................. 32 -

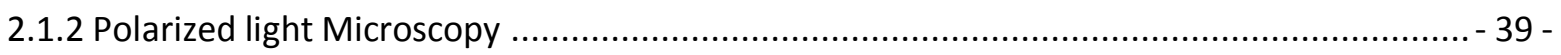

2.1.3 Brewster's Angle Reflection Ellipsometry …............................................................ 42 -

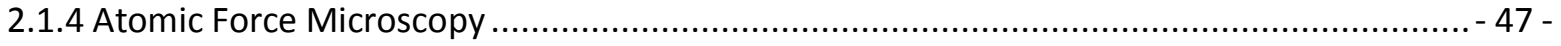

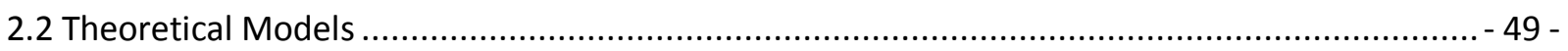

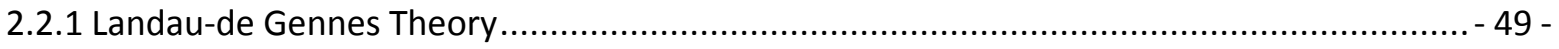

2.2.2 Modeling of the Nematic Surface Order .................................................................... 50 -

Chapter 3. Surface Order at Surfactant-Laden Interfaces between Isotropic Liquid Crystals and Liquid

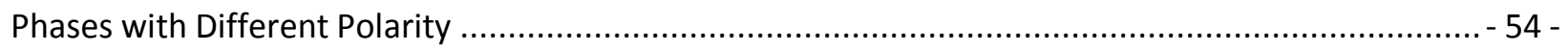




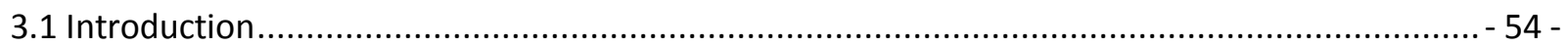

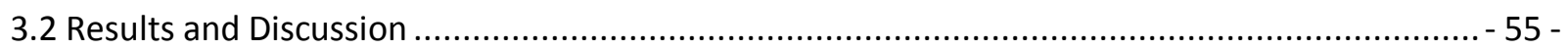

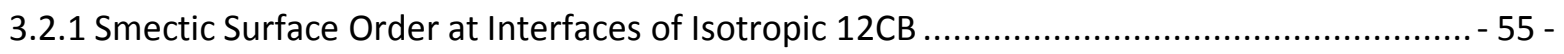

3.2.2 Prewetting Behavior at Interfaces of Isotropic 9СВ ................................................. 59 -

3.2.3 Quantitative Discussion of the Influence of the Glycerol Content ................................... 59 -

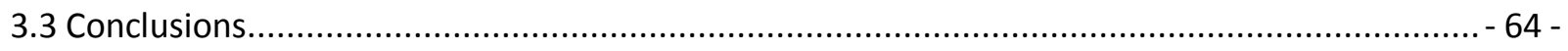

Chapter 4. Surface Ordering and Anchoring Behavior at Liquid Crystal Surfaces Laden with

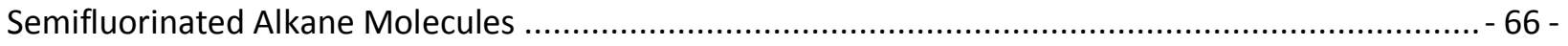

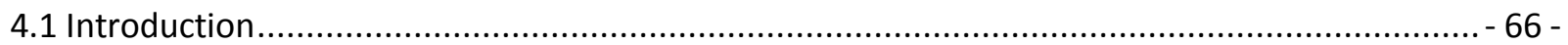

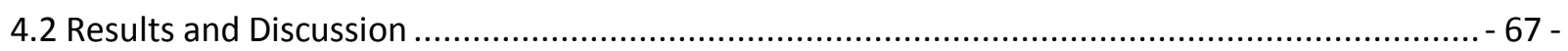

4.2.1 Smectic Surface Order of 12CB Doped with H18F12 …............................................. 68 -

4.2.2 Nematic Surface Order of 8CB Doped with H18F12 …............................................... 72 -

4.2.3 Surface Anchoring of Liquid Crystal Films Laden with H18F12 Molecules ......................... - 77 -

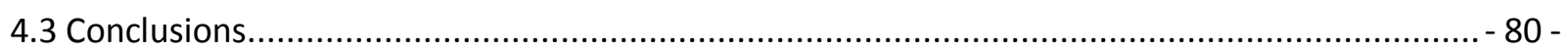

Chapter 5. AFM Visualization of Gibbs Films of Semifluorinated Alkanes at Liquid Crystal/Air Interfaces...82 -

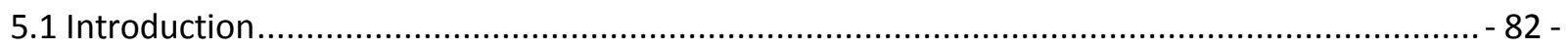

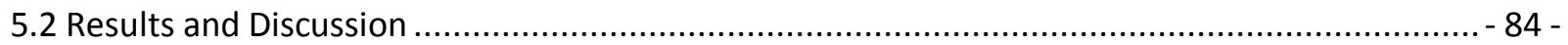

5.2.1 AFM Measurements on Surfaces of Liquid Crystal Droplets Laden with SFA Molecules...... - 84 -

5.2.2 AFM Measurements on the Precursor Film Spreading from a 12CB Droplet Doped with

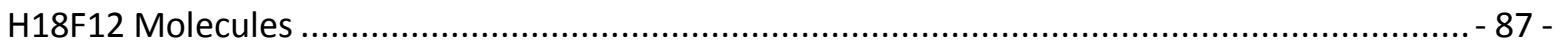

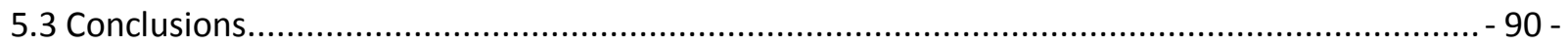

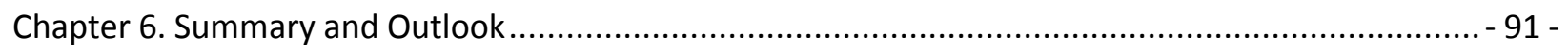

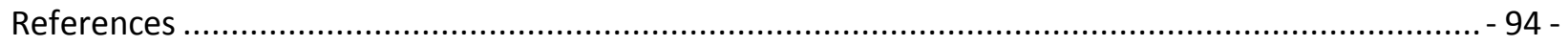

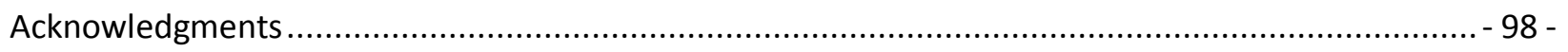

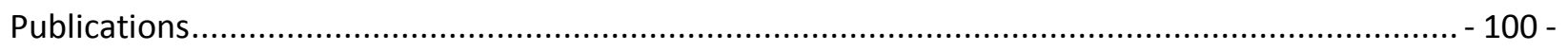

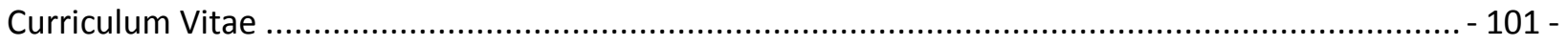




\begin{abstract}
This dissertation concerns research on the structures and self-organization at liquid crystal interfaces laden with surface-active molecules. Of particular interest are the surface ordering phenomena occurring at interfaces formed between liquid crystals and a second fluid or between liquid crystals and air. A major part of the research has been dedicated to exploring the influence of surface-active molecules on the surface-induced pretransitional order and surface anchoring behavior at interfaces of liquid crystals. First, smectic surface layering transitions and nematic prewetting behavior have been investigated at surfactant-laden interfaces between isotropic liquid crystal phases and a water/glycerol phase whose polarity can be systematically varied by the water-to-glycerol ratio. It is shown that the polarity of the immiscible liquid phase can strongly influence the surface coverage of the surfactant molecules and hence the surface ordering behavior of liquid crystals. Second, surface properties of liquid crystal/air interfaces can be manipulated by the adsorption of a special kind of surface-active molecules, i.e., semifluorinated alkanes. The absorbed layer (also called the Gibbs film) of the semifluorinated alkane $\mathrm{C}_{18} \mathrm{H}_{37}-\mathrm{C}_{12} \mathrm{~F}_{25}$ undergoes at the liquid crystal/air interface a structural phase transition from a dilute state at higher temperatures to a dense state at lower temperatures. The structural phase transition causes an anchoring transition from homeotropic to planar of the liquid crystals at the air interfaces. If this transition occurs in the isotropic temperature range of the liquid crystals, it destroys the smectic surface order or changes the orientation of the nematic surface order. This dissertation further describes in situ AFM measurements on the self-organized structures formed in the Gibbs film of $\mathrm{C}_{18} \mathrm{H}_{37}-\mathrm{C}_{12} \mathrm{~F}_{25}$ at the liquid crystal/air interface. It is revealed that the Gibbs film of $\mathrm{C}_{18} \mathrm{H}_{37}-\mathrm{C}_{12} \mathrm{~F}_{25}$ is not featureless but consists of ordered hexagonal surface micelles which are similar to the Langmuir films formed by semifluorinated alkanes.
\end{abstract}




\section{Kurzzusammenfassung}

Die vorliegende Arbeit beschäftigt sich mit dem Einfluss von grenzflächenaktiven Molekülen auf die Struktur und die Selbstorganisation von Flüssigkristalloberflächen. Von besonderem Interesse sind dabei die Ordnungsphänomene, welche an der Grenzfläche zwi-schen Flüssigkristallen und einer zweiten Flüssigkeit sowie zwischen Flüssigkristallen und Luft auftreten. Insbesondere wird die grenzflächeninduzierte flüssigkristalline Ordnung in der isotropen Phase und die Verankerung (Orientierung) der Flüssigkristallmoleküle an der Grenzfläche betrachtet.

Im ersten Teil der Arbeit wird die Grenzfläche zwischen nematischen oder smektischen Flüssigkristallen und wässrigen Phasen, bestehend aus Glycerin und Wasser, untersucht. Die Flüssigkristallphase enthält ein Tensid in konstanter Konzentration, während die Polarität der wässrigen Phase über das Glycerin/Wasser-Verhältnis systematisch variriert wird. Es konnte gezeigt werden, dass eine Erniedrigung der Polarität der wässrigen Phase das Ordnungsverhalten der nematischen oder smektischen Flüssigkristalle in ähnlicher Weise beeinflusst wie eine Erniedrigung der Tensidkonzentration in der Volumenphase.

Der zweite Teil der Arbeit beschäftigt sich mit dem Einfluss von semifluorinierten Alkanen auf das Ordnungs- und Verankerungsverhalten an der freien Oberfläche von smek-tischen und nematischen Flüssigkristallen. Die verwendete Verbindung $\left(\mathrm{C}_{18} \mathrm{H}_{37}-\mathrm{C}_{12} \mathrm{~F}_{25}\right)$ bildet an der Flüssigkristall/Luft-Grenzfläche einen Gibbs-Film aus, der zudem einen strukturellen Phasenübergang von einem verdünnten Zustand bei höheren Temperaturen zu einem dichten Zustand bei niedrigeren Temperaturen aufweist. Der Phasenübergang im Gibbs-Film bewirkt einen Wechsel von senkrechter zu paralleler Verankerung des Flüssigkristalls an der Grenzfläche. Wenn dieser Übergang im Temperaturbereich der isotropen Phase der Flüssigkristalls auftritt, zerstört er die smektische Ordnung oder ändert die Orientierung der nematischen Ordnung an der Oberfläche.

Im dritten Teil der Arbeit werden schließlich Rasterkraftmikroskopie-Untersuchungen des dichten Zustandes des Gibbs-Films von $\mathrm{C}_{18} \mathrm{H}_{37}-\mathrm{C}_{12} \mathrm{~F}_{25}$ an der Flüssigkristall/Luft-Grenzfläche beschrieben. In dieser wohl ersten In-situ-AFM-Untersuchung eines Gibbs-Films konnte gezeigt werden, dass der Gibbs-Film eine selbstorganisierte laterale Strukturierung aufweist, die aus 
einer hexagonaldichten Packung von Oberflächenmizellen besteht. Ähnliche Oberflächenmizellen wurden in übertragenen Langmuir-Filmen von semifluorinierten Alkanen beobachtet. 


\section{Introduction}

Fluid interfaces have special physical and chemical properties, which provide a fascinating platform for exploring many aspects of condensed matter [1-5]. Because of the asymmetry in forces, the structure of a boundary region usually differs from the bulk. Much experimental and theoretical work has been dedicated to investigating the structures and self-organization at interfaces of various liquid systems such as aqueous solutions, metal melts, ionic liquids, alkanes, liquid crystals, and so on. Among a variety of studies, one specific topic concerns the molecular orientation and ordering at fluid interfaces [3-4]. The presence of an interface may exert anisotropic effects on the molecular ordering of the adjacent liquid phase. For instance, one may find that the surface region of a liquid is more ordered than the bulk phase. This situation is referred to as surface ordering or surface freezing. The available examples for such a phenomenon include the surface freezing of normal alkanes and similar chain molecules [6-7], the surface layering in liquid metals [8] and ionic liquids [9], and the nematic or smectic surface order in the isotropic phase of thermotropic liquid crystals [10-12]. The surface-induced pretransitional order of liquid crystals represents a typical type of surface ordering phenomena. At temperatures several degrees above the clearing point, the surface of an isotropic liquid crystal is found to be wet by a thin nematic or smectic layer. In particular when the bulk phase becomes the liquid crystalline state, the perturbation of the order of the liquid crystal molecules at the interfacial region may extend to the bulk. The phenomenon of macroscopic orientation of liquid crystals by surfaces is generally called surface anchoring [13].

Since the discovery in 1888 , liquid crystals have attracted great scientific interests because of the richness of the associated physical phenomena [14-17]; and the ubiquitous applications of liquid crystal technologies nowadays, mainly based on their excellent electro-optical properties [18-19], have been a driving force for numerous studies on liquid crystals over the past two decades. The physics and material properties of liquid crystals at interfaces have been one of the most important aspects in liquid crystal research [20]. While the knowledge of liquid crystal/solid interfaces may be in the state of maturity partly due to the crucial importance in 
liquid crystal displays [21], more efforts are still needed to make in order to understand the properties of liquid crystals interfaced to fluids or air. In contrast to most of the investigated liquid crystal/solid interfaces which are rigid, the fluid or air interfaces are obviously soft and hence deformable. The advantage of deformability shown in soft liquid crystal interfaces has been utilized to construct new confinement systems for liquid crystal research, which includes liquid crystal droplets [22-25], shells [26-27], and tubes [28], air bubbles in liquid crystal matrices [29], and liquid droplets at liquid crystal surfaces [30-31]. In these systems, the interfacial properties often play a crucial role in determining many physical or chemical processes.

In recent years, Abbott, Schwartz and co-workers have designed functional interfaces between liquid crystals and aqueous solutions. The interfaces were laden with synthetic or natural amphiphiles (surfactants and lipids), proteins and polymers which served to modify the interfacial interactions between the liquid crystals and water [22, 24, 32-40]. Such interfaces can be extremely robust since a wide spectrum of molecules can be chosen to vary the interfacial properties. This system opens up a new path to biological applications of liquid crystals and establishes a feasible model for study of biophysical problems [33]. Meanwhile, Bahr has experimentally investigated surface-induced pretransitional order at isotropic liquid crystal/water interfaces laden with surfactants [41-43]. By tuning the strength of the surface field, novel surface-induced ordering behaviors which were predicted by theoretical models have been observed. In these previous studies, the liquid crystals under investigation were interfaced to a pure water phase or a water phase doped with a small amount of amphiphiles. However, immiscible fluids apart from water may be useful in many real situations, e.g., microemulsions and microfluidics. Thus, it is necessary to study the surface anchoring and surface-induced pretransitional ordering behavior of liquid crystals interfaced to non-water fluids or fluids in which water is just one of several components.

In the experimental systems developed by Abbott, Bahr, Schwartz and co-workers, the orientation of the liquid crystals is controlled by the self-assembly of surfactants, lipids, proteins or synthetic polymers at the aqueous interfaces. For instance, anchoring transitions from homeotropic to planar, or vice versa, could be achieved by changing the characteristics of the surface-active molecules, e.g., surface coverage and molecular geometry [33, 36, 41-43]. The 
dynamic processes of these interfacial molecules such as adsorption, desorption and phase transitions, in turn, could be monitored by the anchoring behavior of the liquid crystals in contact. Therefore, investigating the liquid crystal interfaces laden with surface-active molecules can enrich the understanding of molecular self-assembled behavior.

Liquid crystal/air interfaces on the one hand can be regarded as the simplest liquid crystal interfaces and hence were studied rather early, for example, by measuring the surface tension one century ago [44]. Surface tension measurements of the isotropic phase of a nematic liquid crystal indicated for the first time the presence of pretransitional surface order [44]. Besides, surfaceinduced smectic order was also first found at the air interface of a smectic liquid crystal in the bulk isotropic temperature range [11]. On the other hand, manipulation of the properties at the liquid crystal/air interfaces seems to be difficult since common surfactants, basically designed for water, cannot be absorbed to the air interface of liquid crystals. For some rod-like liquid crystals (e.g., alkylcyanobiphenyl liquid crystals), the anchoring of the molecules at the air interface has been found to be always homeotropic (the molecular axis being perpendicular to the interface) [44-45]. Until now, no reports have ever shown a surface functionalization method to change the anchoring of liquid crystals at the air interface. Correspondingly, it has been also unknown so far whether or not the rich types of wetting behavior and phase transitions found at the isotropic liquid crystal/water interfaces [41-43] can be extended to the air interface. Furthermore, new ordering phenomena might occur at the air interfaces considering the fact that interactions therein are much simpler than those at the aqueous interfaces.

In order to address the abovementioned open questions, the research described in this dissertation extends the previous concept of surface ordering and anchoring at liquid crystalwater interfaces to that at interfaces between liquid crystals and air or other immiscible fluids. It is shown that the surface ordering and anchoring behavior of liquid crystals at interfaces can be dramatically influenced by changing the bulk properties of the immiscible fluids or controlling the self-assembly of the amphiphiles at the interfaces.

This dissertation is organized in the following manner. In the first Chapter, the basic knowledge of liquid crystals that is correlated to this research is presented. Moreover, surface anchoring of liquid crystals, surface ordering at liquid interfaces, and surface-induced pretransitional order of isotropic liquid crystals are also introduced. Chapter 2 describes the 
principles of the employed experimental techniques and the theories for the interpretation of the obtained experimental results. Polarizing microscopy, ellipsometry and atomic force microscope (AFM) are the three techniques used in this research. Concerning the theoretical aspect, Sheng's Landau model [46] for interpreting the nematic surface order are described in this chapter. Chapter 3 is dedicated to the investigation of surface order at surfactant-laden interfaces between isotropic liquid crystals and liquid phases with different polarity. It is shown that changing the polarity of the immiscible fluid interfaced to the liquid crystals leads to different surface ordering behavior. Chapter 4 is focused on liquid crystal/air interfaces. The liquid crystals were doped with the semifluorinated alkane $\mathrm{C}_{18} \mathrm{H}_{37}-\mathrm{C}_{12} \mathrm{~F}_{25}$ that is able to be absorbed to the liquid crystal/air interface and hence modify the property of the liquid crystal/air interface. In particular, the absorbed layer of the semifluorinated alkane itself shows at the interface a structural phase transition from a dilute state at higher temperatures to a dense state at lower temperatures [47]. The influence of the dynamic structure of the absorbed semifluorinated alkane layer on the surface ordering and anchoring behavior is presented. In the study shown in Chapter 5, AFM is employed to unveil the organized structures of the semifluorinated alkane $\mathrm{C}_{18} \mathrm{H}_{37}-\mathrm{C}_{12} \mathrm{~F}_{25}$ absorbed at the surface of smectic liquid crystals. Previous studies by surface sensitive scattering techniques have revealed that a dense packing of the molecules exists at the surface of hydrocarbon liquids at lower temperatures [47]. The AFM characterization further demonstrates that the dense packing is not featureless but consists of self-assembled hexagonal patterns. Finally, a summary and an outlook on the surface ordering and anchoring at liquid crystal interfaces laden surface-active molecules are made in the last Chapter. 


\section{Chapter 1. Research Background}

\subsection{Liquid Crystals}

\subsubsection{Liquid Crystal: the Forth State of Matter}

Our everyday experience tells us that there exist three standard states of matter - vapor, liquid and solid. For example, water vapor can be observed in the form of steam when water is boiled in a pot; the vapor can condense into the liquid state of water by forming drops; if these water drops are put in a freezer, they would transform into solid pieces of ice. Being either crystalline or amorphous, a solid has a stable, definite shape. Unlike solids, liquids can flow and adopt the shape of their containers. In addition, an important property that differentiates crystalline solids from liquids is the anisotropy which arises from the long-range order of the constituent molecules. This difference can be illustrated in many bulk properties, for instance, the optical properties: the refractive index of a simple liquid is independent of the direction of the incident light, but a crystalline solid is anisotropic and the refractive index depends on the direction of light propagation, the property of which is known as birefringence.

The term "liquid crystal" may be puzzling, but it accurately defines a special phase of matter. Liquid crystals are partially ordered fluids which are intermediate between isotropic liquids and crystalline solids. Therefore, the term "mesophase", originating from the Greek word meso (in between), is also used to describe the subphases of liquid crystalline materials. Correspondingly, compounds that produce mesophases are called "mesogens". The physical properties of liquid crystalline materials exhibit characteristics of both liquids and crystalline solids[15-16]. For instance, liquid crystals can flow because their molecules possess a level of mobility much similar to the molecules of isotropic liquids. On the other hand, orientational order and sometimes positional order can be found in liquid crystals even though the degree of order is small compared to that of crystalline solids. One finds a tendency of liquid crystal molecules to point along a common axis, called the director. Thus, liquid crystals are anisotropic fluids: for 
example, they are birefringent materials that can decompose light into two rays like crystalline solids. Depending on the ways in which the transitions to the mesophases may be brought about, liquid crystals are generally divided into two generic classes: lyotropic and thermotropic liquid crystals. Lyotropic liquid crystals are multi-component systems and their phase behavior is essentially influenced by the composition and the temperature [48]. Regarding thermotropic liquid crystals, the mesophases usually form in single compounds by a change of temperature and are stable for a certain temperature interval. Since the research presented in this dissertation concerns only thermotropic liquid crystals, the discussion is restricted to this type of liquid crystals.

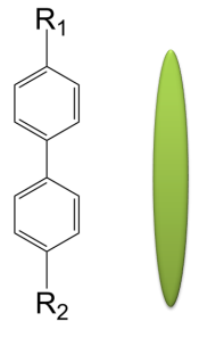

calamitic

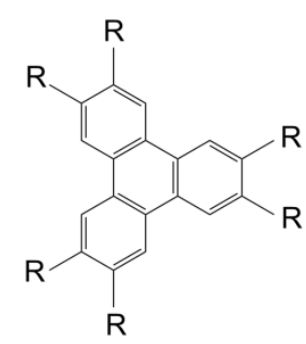

discotic

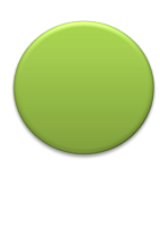$$
\text { . }
$$

Figure 1.1 Schematic illustrations of calamitic and discotic liquid crystal molecules.

(a) $8 \mathrm{CB}$

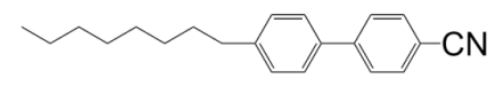

(b) $9 \mathrm{CB}$

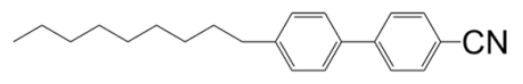

(c) $12 \mathrm{CB}$

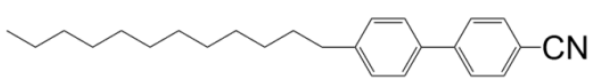

(d) MBBA

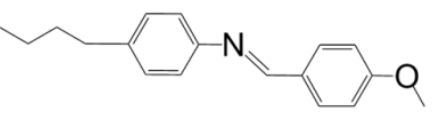

Figure 1.2 Molecular structures of typical calamitic mesogens. (a, b, c) Three homologues of alkylcyanobiphenyl liquid crystals (nCB); (d) N-(4-methoxybenzylidene)-4-butylaniline (MBBA).

Although the liquid crystalline state does not fall into the standard definition of states of matter, it can be widely found in many synthetic or even natural materials. In fact, the first 
reported observation of liquid crystalline phase, by the Austrian botanical physiologist Friedrich Reinitzer in 1888, was from the derivatives of cholesterol that are essential for all animal life [49]. Materials possessing thermotropic liquid crystalline phases have a common characteristic, i.e., their molecules are highly anisotropic. According to the shape of the constituent molecules of the mesophases, one can group thermotropic liquid crystals into two main types: calamitics (rod-like) and discotics (disc-like), as shown in Figure 1.1. In a calamitic liquid crystal, one often finds a common rigid core (phenyl or biphenyl groups) attached to one or two flexible endgroups (alkyl or alkoxy chains). This class of liquid crystals has been intensively investigated and used for all commercially available liquid crystal displays nowadays. Figure 1.2 shows the structure of typical calamitic molecules, i.e., three homologues of the alkylcyanobiphenyl liquid crystals and MBBA. The alkylcyanobiphenyl compounds are commonly called nCB (n denotes the carbon number in the alkyl chain). All the liquid crystalline compounds used in this study are the homologues of $\mathrm{nCB}$. Concerning the discotic molecules, six or eight flexible endgroups are linked to a rigid, disc-like core, as schematically illustrated in Figure. 1.1. This class of liquid crystals was discovered by Chandrasekhar et al. in 1977 [50].

\subsubsection{Thermotropic Liquid Crystalline Phases}

In the history, the research of liquid crystals began in 1888 with the observation of the temperature-dependent phase behavior of a material known as cholesteryl benzoate. When Friedrich Reinitzer increased the temperature of a solid sample, he discovered that this compound had two distinct melting points [49]. Upon heating, this compound first transformed from a crystal to a turbid liquid, and then by increasing the temperature further, it changed again into a clear liquid. On cooling the transparent liquid, Reinitze found that this sequence was reversed. The turbid liquid observed by Reinitzer was in the nematic phase, more precisely, the chiral nematic phase. Since the liquid crystal compounds used in our study do not possess any chiral centers in their molecules, we leave aside our discussions on chiral liquid crystalline phases. 


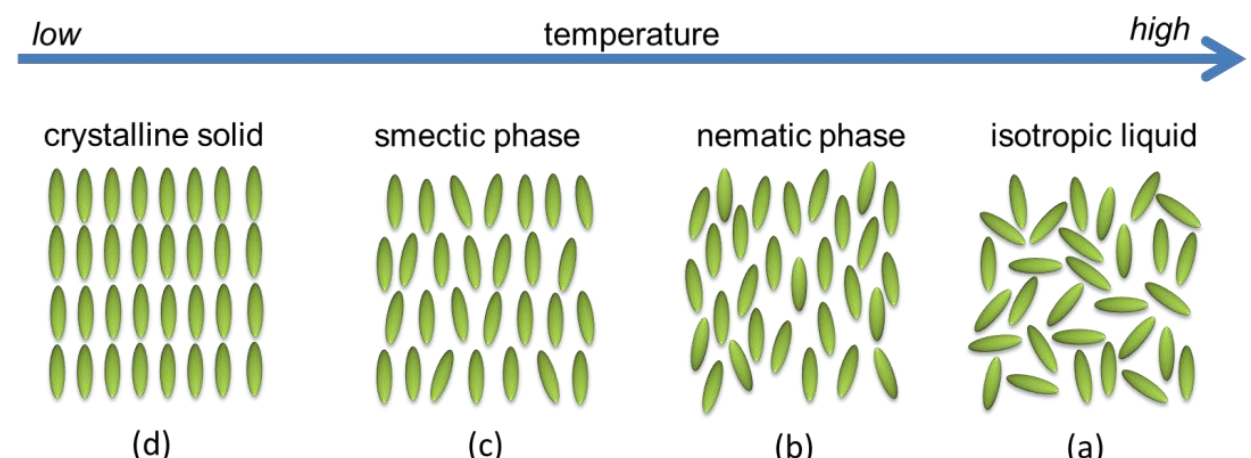

Figure 1.3 Schematic illustration of the phase sequence of (a) isotropic liquid, (b) nematic, (c) smectic- $A$ and (d) crystalline phases. The axis denotes the direction of increasing temperature.

Thermotropic mesophases occur in a certain temperature range and hence the temperature is the most essential variable of state. At too high temperatures, the regular arrangement of molecules is broken by thermal motions, giving rise to totally disordered liquids with the loss of orientational and positional order (see Figure 1.3a). If the temperature is too low, most liquid crystalline materials will form a conventional crystal (see Figure 1.3d). The nCB compounds display a typical phase sequence of liquid crystalline materials. At lower temperatures, they are crystalline solids as common substances, with both positional and orientational order of molecules. Above the melting point, instead of melting directly into clear liquids, the compounds of nCB may undergo phase transitions into either nematic $(\mathrm{N})$ or smectic- $A(\mathrm{SmA})$ phases which appear to be hazy by a casual observation. As schematically shown by Figure 1.3b, the nematic phase is the most common liquid crystalline phase, where only the long-range orientational order of the long molecular axis is present in the bulk without any positional order. Upon lowering the temperature of a nematic material, the smectic- $A$ phase may appear. Apart from the long-range orientational order, this mesophase possesses the positional order in one spatial direction which can be regarded as a layered structure (see Figure 1.3c). Originally named by Friedel in 1922 [17, 51], nematic and smectic phases are the two most important and frequently studied mesophases that are exhibited by non-chiral calamitic molecules. In fact, the nomenclature of these two mesophases was based on their optical textures. The texture of optically extinct threads is the one that led to the name of nematic phase ("nematic" is a Greek word meaning thread-like). Likewise, the smectic phase appeared to be soap-like and is hence named as by the Greek word "smectic". 
The molecular structure of liquid crystals determines the specific phases at the specific temperatures, as well as the phase sequences. A great number of liquid crystalline compounds with tailored structures have been synthesized under the consideration of both fundamental research and practical applications. In addition to nematic and smectic- $A$ phases, these designed liquid crystalline materials may manifest other mesophases, such as, cholesteric phase, blue phase, twist grain boundary phase, etc. Some liquid crystalline compounds may exhibit only one single mesophase during the thermo-process, but many of them do exhibit two or three different types of liquid crystalline phases.

\begin{tabular}{|c|c|c|c|c|c|c|c|c|}
\hline \multicolumn{2}{|c|}{ nCB compounds } & \multicolumn{7}{|c|}{ transition temperature $\left({ }^{\circ} \mathrm{C}\right)$} \\
\hline name & molecular formula & crystalli & & $\mathrm{SmA}$ & & $\mathrm{N}$ & & isotrpic \\
\hline $5 \mathrm{CB}$ & $\mathrm{C}_{5} \mathrm{H}_{1}$ & $x$ & 22.5 & - & & $x$ & 35.0 & $x$ \\
\hline $8 \mathrm{CB}$ & $\mathrm{C}_{8} \mathrm{H}_{1}$ & $x$ & 21.0 & $x$ & 33.5 & $x$ & 40.5 & $x$ \\
\hline $9 \mathrm{CB}$ & $\mathrm{C}_{9} \mathrm{H}_{19}$ & $x$ & 42.0 & $x$ & 48.0 & & 50.0 & $x$ \\
\hline $10 \mathrm{CB}$ & $\mathrm{C}_{10} \mathrm{H}_{21}$ & $x$ & 44.0 & $x$ & 50.5 & - & & $x$ \\
\hline $11 \mathrm{CB}$ & $\mathrm{C}_{11} \mathrm{H}$ & $x$ & 53.0 & $x$ & 57.0 & $x$ & 57.5 & $x$ \\
\hline $12 \mathrm{CB}$ & $\mathrm{C}_{12} \mathrm{H}_{23}-$ & $x$ & 48.0 & $x$ & 59.0 & - & & $x$ \\
\hline
\end{tabular}

Table 1.1 Phase sequences and transition temperatures of alkycyanobiphenyl (nCB) homologues. Cross $(X)$ and dash ( - ) denote the existence and nonexistence of a specific phase, respectively.

Let us look at the homologues of some selected $\mathrm{nCB}$ mesogens which can show nematic or smectic- $A$ phases. By a careful examination of their structure, we can see that the nCB compounds contain a cyano group which contributes to the positive dielectric anisotropy and hence a high optical anisotropy. Simply by a change of the chain length of the alkyl groups, their phase sequences and transition temperatures are varied, as presented in Table 1.1. If the nCB compounds (e.g., $8 \mathrm{CB}, 9 \mathrm{CB}$, and $11 \mathrm{CB}$ ) possess both the nematic and the smectic- $A$ phases, they must follow a phase sequence of isotropic-nematic-smectic-crystalline when the temperature is decreased from above. Moreover, it is also possible that some nCB mesogens have a single mesophase, either the nematic phase or the smectic- $A$ phase: for example, $5 \mathrm{CB}$ melts into the 
nematic phase when heated above its melting point, whereas 10CB and 12CB display only the smectic- $A$ phase upon cooling from the isotropic phase. It is difficult to make a very simple rule of molecular structures that can governs the generation of the types of mesophases, since both the steric and polarity considerations have to be taken into account. However, experience has revealed that the generation of a nematic phase is facilitated by inducing relatively short terminal chains, whereas the molecular structure of smectic mesogens must be more symmetrical and allow for lateral intermolecular interactions to stabilize the lamellar arrangement of molecules [52].

\subsubsection{Structure and Properties of Thermotropic Liquid Crystals}

Before describing the structure and properties of the nematic and smectic phases, we should introduce two indispensable parameters in the liquid crystal research: the director $\vec{n}$ and the order parameter $S$ [14]. These two concepts are of vital importance in the research described in this dissertation and will be frequently mentioned in the following.

(a)

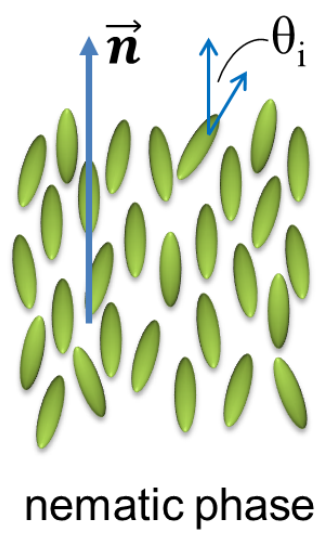

(b)

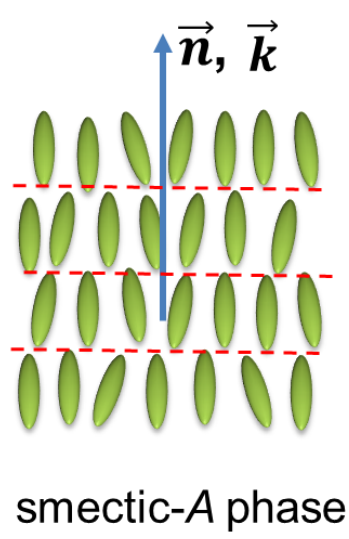

Figure 1.4 Schematic illustrations of the director $\vec{n}$ in the nematic phase (a) and the smectic- $A$ phase (b). $\theta_{i}$ in (a) denotes the angles of the individual molecules with $\vec{n}$; $\vec{k}$ in (b) is the surface normal of the smectic layers.

Even though the molecules in liquid crystals can diffuse as those in isotropic liquids, the long molecular axis prefers to point along a common direction, allowing the liquid crystal phases to maintain some degree of orientational order. This common direction, called the director $\vec{n}$ (a unit 
vector), denotes the spatial and temporal average of the long molecular axis. The sign of $\vec{n}$ is of no importance since in most liquid crystals both directions of the vector $\vec{n},+\vec{n}$ and $-\vec{n}$, are equivalent. The nematic phase is the simplest liquid crystalline phase, exhibiting only orientational order. In the nematic phase, as schematically illustrated in Figure 1.4a, whereas the mass centers of the molecules undergo diffusion isotropically in all three dimensions, the molecular axes show an angular distribution with respect to the director $\vec{n}$. For the smectic phases, they are more ordered than the nematic phase and contain lamellas in their structure. The director $\vec{n}$ in the smectic- $A$ phases is perpendicular to the smectic layers or parallel to the smectic layer normal $\vec{k}$ (see Figure $4 \mathrm{~b}$ ). Furthermore, in the case of a nematic and a uniaxial smectic liquid crystal, the director also gives the optical axis, which will be discussed in Chapter 2 .

In order to quantify the amount of the orientational order in the mesophase, one often needs to use the scalar order parameter $S$. The order parameter is zero in the isotropic phase and 1 in the phase with perfect parallel order. Then, we have $0<S<1$ in the liquid crystalline phase. The order parameter $S$ is defined as

$$
S=\frac{1}{2}<3 \cos ^{2} \theta_{i}-1>
$$

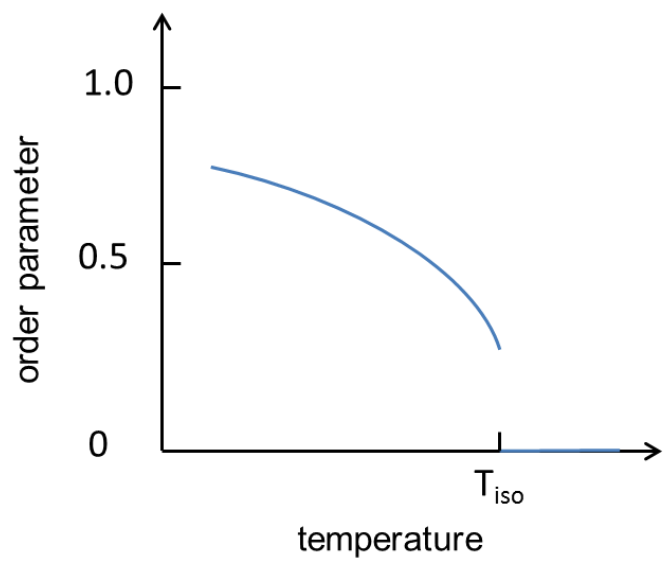

Figure 1.5 Scalar order parameter $S$ vs temperature: $\mathrm{T}_{\text {iso }}$ is the isotropic-liquid crystalline transition temperature. 
where $\theta_{i}$ is an angle between the individual molecular long axis and the director $\vec{n}$ and the brackets indicate both the temporal and spatial average [14]. For typical liquid crystals, the orientational order parameter is a decreasing function of temperature (see Figure 1.5), possessing values between 0.3 and 0.8 . The measurement of the orientational order parameter is one of the main topics in fundamental research on liquid crystals. Many techniques have been applied to determine the order parameter, such as nuclear magnetic resonance (NMR) [53], Raman scattering [54], fluorescence [55], infrared spectroscopy [56], and so on. In those measurements, the order parameter can be directly associated to some experimentally measurable quantities, e.g., birefringence, susceptibility, dielectric anisotropy, etc. In my research, the order parameter profile at the liquid crystal interfaces will be transformed into the refractive index profile.

The concept of order parameter is also of physical importance in the heart of every phenomenological theory of phase transitions. For instance, the Landau-de Gennes theory, applied to describe the isotropic-nematic transition, assumes that the order parameter in the nematic phase is small in the vicinity of the transition and hence the free energy of the system can be expanded in a power-law series of the order parameter [15]. To theoretically interpret the observed nematic pretransitional order at surfaces in our experiment, the concept of order parameter will be emphasized again in Chapter 2 where Sheng's Landau-de Gennes model [46] for describing the surface-induced pretransitional order of nematic liquid crystals is discussed.

The orientational order in liquid crystals results in the anisotropy, which further distinguishes them from common liquids. Therefore, some of their properties (known as tensor properties) depend on the direction along which they are measured [16]. Nematic and smectic- $A$ phases are uniaxial phases because all directions perpendicular to the director are equivalent but different from the director. The anisotropy can be illustrated in the electric, magnetic, optical, and elastic properties. In principle, one can observe the anisotropic response of a liquid crystal to an external force or perturbation, such as an electric field, a magnetic field, and a mechanical force. For example, because of the anisotropic of the dielectric susceptibility, liquid crystals show the ability to align the director along an external electric field. Such a property has been utilized in industries for liquid crystal displays [19]. Magnetic fields have the analogous influences on alignment of liquid crystals as electric fields. Nevertheless, even in the absence of any of the abovementioned external fields, the director of a liquid crystal can be forced to point in a specific 
direction by introducing an outside surface to the system. The effect from the surface is called surface anchoring, which is one main theme of the dissertation and will be discussed in a more detailed way in Section 1.2.

In some sense, investigation of electric, magnetic, optical, and elastic properties of liquid crystals requires sophisticated experimental instruments and skills. In the first step, one can distinguish, simply by naked eyes, whether the liquid crystal sample is in the isotropic phase or in the liquid crystalline phase. The reason is that a non-aligned liquid crystal sample (when in the liquid crystalline state) appears to be turbid due to the fluctuation of the director whereas an isotropic liquid looks transparent. Furthermore, it might be also easy to detect in which mesophase (i.e., nematic or smectic- $A$ ) the same liquid crystal sample turns out to be, because the smectic- $A$ phase is more viscous and opaque than the nematic phase for the same sample. Reasonably, it is convenient to put the sample under an optical microscope so as to obtain more information.

Identifying the texture of mesophases under an optical microscope is a simple, straightforward method for studying the structure of liquid crystals [51]. A polarizing microscope along with a temperature controller can determine both of the types of mesophases and their transition temperatures, which basically provides useful information about the properties of the mesogens. Usually, a thin sample of a liquid crystalline material is prepared on glass slides. Figure 1.6 shows the polarizing microscopy images of $8 \mathrm{CB}$ in the nematic phase and the smectic- $A$ phase, respectively. The texture shown in Figure 1.6a that is formed by sandwiching a nematic 8CB drop between two glass slides is a characteristic Schlieren texture for the nematic phase. The classical smectic- $A$ phase can form on cooling the nematic phase or directly from the isotropic phase. Figure 1.6b shows the typical focal conic texture with Maltese crosses from a smectic- $A$ film on the glass substrate. The observed textures are strongly correlated to the orientation of the director. In both the images of Figure 1.6, since no external field is exerted to the liquid crystal samples, the substrates serve to align the liquid crystal. Thus, by analyzing the texture of the liquid crystal film under the polarizing microscope, one may obtain rich information regarding the anchoring effects from the surfaces. 

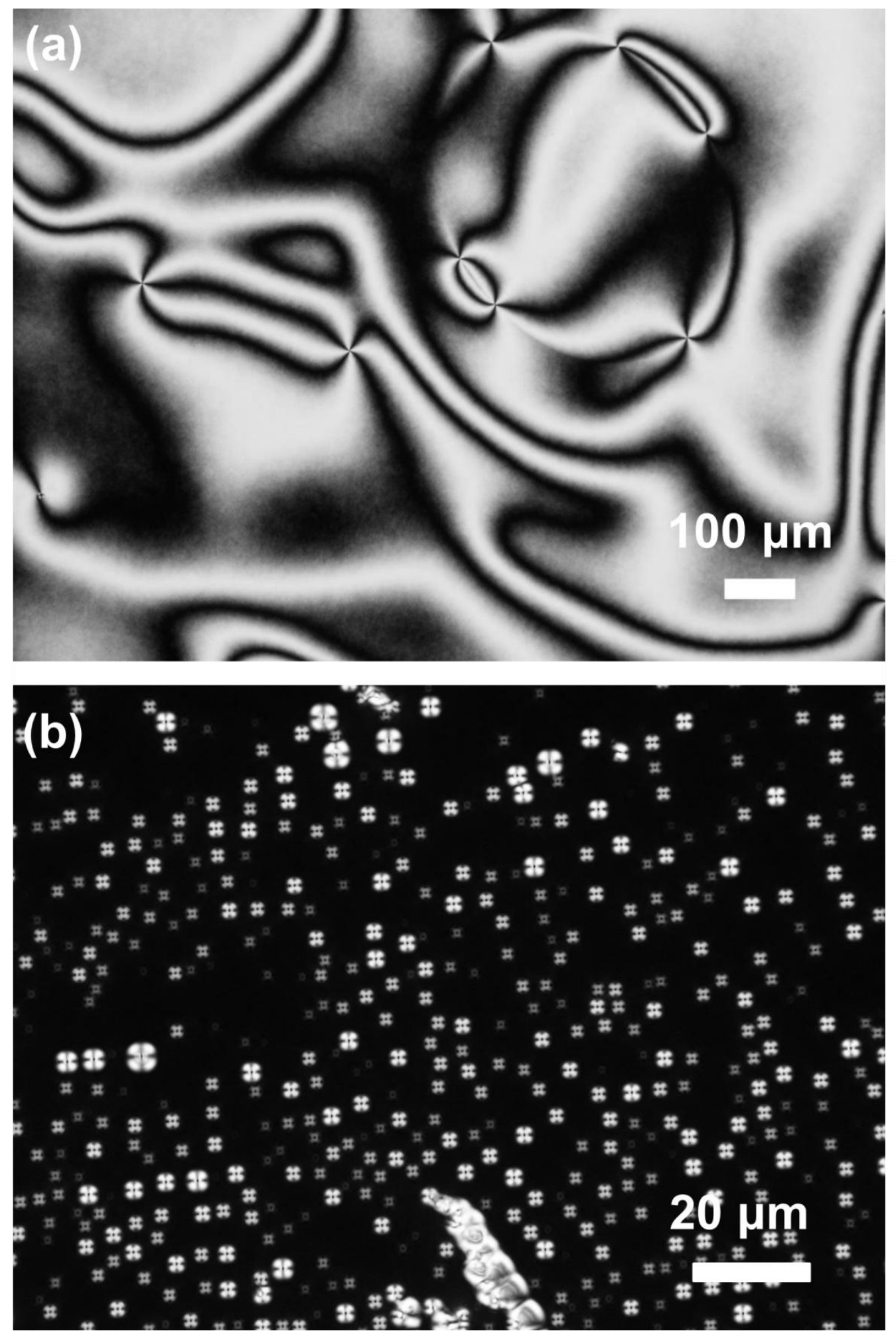

Figure 1.6 Polarizing microscopy images of (a) Schlieren texture of the Nematic phase and (b) Maltese crosses from focal conic domains of the Smectic- $A$ phase. The liquid crystal used was $8 \mathrm{CB}$. 


\subsection{Surface Anchoring of Liquid Crystals}

\subsubsection{Types of Surface Anchoring}

When a liquid crystal is brought in contact with another phase (air, liquid or solid) forming an interface, the director of the liquid crystal can be oriented by the interface. The phenomenon of surface anchoring was actually known one century ago and the alignment of liquid crystals by surfaces forms the basis of many liquid crystal devices nowadays [57]. However, the mechanisms of surface anchoring are not completely understood. The orientation of the liquid crystal molecules is highly sensitive to the surface properties and depends on many factors of the interface such as chemical composition, roughness, charge, elasticity, and so on.

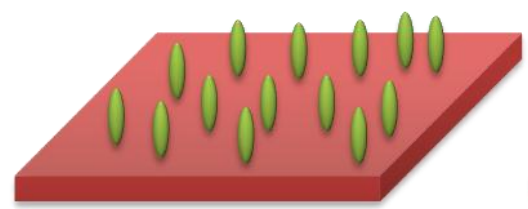

homeotropic

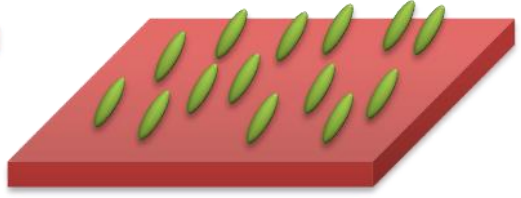

uniform tilted

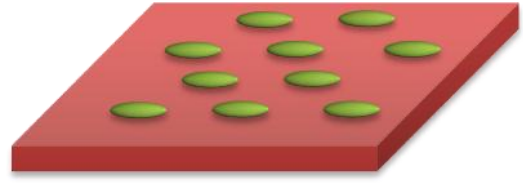

uniform planar

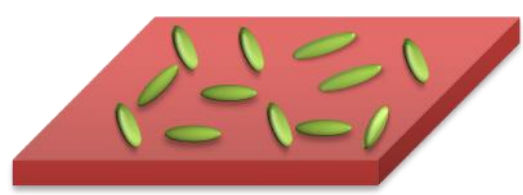

random planar

Figure 1.7 Schematic illustrations of four different anchoring conditions.

In general, four main types of surface anchoring are found, as illustrated in Figure 1.7. When the director $\vec{n}$ of the liquid crystal is aligned perpendicular to the interface, we call such an anchoring condition as homeotropic. Moreover, the director $\vec{n}$ can be uniformly tilted from the surface normal, the situation of which is referred to as uniform tilted. In addition, there are two planar anchoring conditions whereby the director $\vec{n}$ is in parallel with the plane the interface. The uniform planar anchoring is defined as the director $\vec{n}$ pointing along a preferred azimuthal 
direction. Concerning the random planar anchoring, the director $\vec{n}$ is oriented in the plane of the surface without a fixed azimuthal direction.

In all applications of liquid crystal devices as well as fundamental research, the uniform orientation of liquid crystals by surface anchoring is desirable. Thus, many techniques have been developed to achieve this goal. As the nature of surface anchoring is so sensitive to the phase in contact with the liquid crystal, a technique that works successfully for one surface may not be applicable in another surface. For the simplicity, herein, the techniques can be considered according to their applications in two major different types of substrates: rigid substrates (solid) and soft substrates (liquid or air).

\subsubsection{Surface Anchoring at Rigid Interfaces}

First, let us consider solid substrates. In the absence of any surface treatment, the anchoring effect from a bare solid substrate is hard to predict. In order to obtain well-defined surface anchoring conditions, an alignment layer is usually coated on the surface of the solid. The alignment layer can be, for example, a long-chain aliphatic layer, a spin-coated polymer film, or a thermally evaporated $\mathrm{SiO}$ film [21].

The long-chain aliphatic layers, formed either by covalently bonding self-assembled monolayers (SAMs) [58] or by transferring Langmuir-Blodgett (LB) films of surfactants [59$60]$ onto the substrate, are usually employed to generate the homeotropic anchoring. A wellknown aliphatic silane, N, N-dimethyl-N-octadecyl-2-aminopropyltrimethoxysilyl chloride (DMOAP), has been frequently used to chemically modify glass substrates so as to induce the homeotropic anchoring [58]. It has been known that the packing of the DMOAP molecules on the glass substrates is not highly dense which makes it possible for calamitic mesogens to penetrate into the cavities between the aliphatic chains. Regarding the LB films of surfactants, Hiltrop and Stegemeyer have carried out a series of systematic studies on the influences of molecular structure, temperature and packing density on the quality of the homeotropic anchoring [59-60]. One of their findings worthy of mention here is that both the loose packing and the dense packing of the surfactant molecules give rise to random alignment. Only when the molecular packing in the LB film is in the intermediate regime, the homeotropic anchoring can be realized. 
To obtain uniform planar alignment in liquid crystals, one of the feasible methods is mechanical rubbing of a spin-coated polymer film on the substrate. Although this method was developed by Mauguin more than one hundred years ago [57], it still dominates in the alignment process in the current manufacture of liquid crystal devices [21]. In the process, most often a thin film is spin-coated on a solid substrate from the solution of polyimides or nylon. Then, the substrate is unidirectionally rubbed by a cloth with short fibers. The unidirectional rubbing results in the formation of uniform microgrooves on the substrate which has been considered as one of the two mechanisms responsible for the uniform planar anchoring [21]. The other mechanism is believed to be the reorganization of the polymer molecules [20-21].

Random planar anchoring may not be as useful as the uniform planar anchoring in liquid crystal industries, but sometimes for fundamental research this type of alignment is necessary. For instance, in order to obtain the focal conic texture of a smectic film on a solid substrate, the substrate has to induce the random planar anchoring to the smectic film (see also Figure 1.6b). The random planar anchoring can be found on bare silicon substrates (freshly cleaned by Piranha solutions) of silicon substrates coated by polyethyleneimine (PEI) or N-trimethoxysilylpropyl-N, $\mathrm{N}, \mathrm{N}$-trimethylammonium chloride (MAP) [61].

Oblique evaporation of $\mathrm{SiO}$ is one of the non-contact methods for liquid crystal alignment [62]. This method is often used to generate uniform tilted anchoring. During the evaporation process, a micro-columnar structure is formed on the surface of the substrate. By changing the angle between evaporation beam and substrate normal, one can obtain different surface structure of the oblique evaporated film. A tilt angle of up to 20 degree can be achieved by this method [21].

\subsubsection{Surface Anchoring at Soft Interfaces}

A soft liquid crystal interface can be created between a liquid crystal and air or between a liquid crystal and an immiscible fluid (e.g., water). In the early years of liquid crystal research, soft liquid crystal interfaces did not receive much attention from the scientific community compared with solid interfaces. This circumstance has been changed recently due to the development of unconventional applications of liquid crystals in novel self-organization behavior [30-31, 63], photonics [25], and chemical and biological sensing [22, 24, 33-37, 64]. The advantage of deformability in soft liquid crystal interfaces has been utilized to construct new 
confinement systems for liquid crystal research, which includes liquid crystal droplets [22-25], shells [26-27], and tubes [28], air bubbles in liquid crystal matrices [29], and liquid droplets at liquid crystal surfaces [30-31]. From the abovementioned systems, a core question arises how the orientation of the liquid crystals is controlled at soft interfaces.

The simplest soft liquid crystal interface is the air interface (or the free surface) where the liquid crystal does not interact with any substrates. The director of the liquid crystal is oriented along the free-energy-minimizing direction [65]. Depending on the nature of the liquid crystals, the anchoring conditions at the air interface can be varied. Most of calamitic mesogens such as nCB are homeotropically anchored at the air interface [44-45]. A possible reason for this behavior is because the polar head of the molecules tends to point away from the air phase to reduce the surface tension. In contrast, few liquid crystals (e.g., para-azoxyanisole), usually without any long alkyl tail in the molecules, show planar anchoring at the air interface [66]. In addition, the director of some liquid crystals (for example, MBBA) at their free surfaces is tilted from the surface normal and the tilt angle can be dependent on the temperature [65]. Although contaminates or dopants in liquid crystals might be absorbed at the free surface of some liquid crystals, their effects on the intrinsic anchoring of liquid crystals are weak. For example, an alkoxy cyanobiphenyl molecule with a partly fluorinated chain was designed and used by Alexander et al. to dope 5CB [67]. They found that this dopant was strongly adsorbed at the interface between 5CB and air but did not change the surface anchoring of 5CB. Until now, no reports have ever shown a successful method by surface modification to remarkably alter the anchoring at the free surface of liquid crystals. The physical mechanism behind the anchoring behavior at the air interface is complicated and poorly understood. Many theoretical models have been proposed to explain the anchoring at the air interface [68]. Unfortunately, none of them have been able to obtain a satisfactory quantitative agreement with the experimental measurements.

In recent studies, liquid crystals under investigation were in contact with aqueous solutions forming an interface. The bare surface of pure water usually imposes random planar anchoring to nematic [32, 41] or smectic liquid crystals [42]. In order to turn the alignment into homeotropic, the interface can be chemically or biologically functionalized by self-assembly of surfactants, lipids, proteins, and synthetic polymers [35]. A typical model of these systems is an interface 
laden with surfactant molecules consisting of a polar head (hydrophilic) and an aliphatic tail (hydrophobic), as schematically illustrated in Figure 1.8. The surfactant can be dissolved either in the water phase [37] or in the liquid crystal phase [42-43]. Several conventional surfactants, such as alkyltrimethylammonium bromide $\left(\mathrm{C}_{\mathrm{n}} \mathrm{TAB}\right)$ [41], sodium dodecyl sulfate (SDS), 1-(cis9-octadecenoyl)-rac-glycerol (Monoolein) [42-43], and polyoxyethylene alkyl ethers $\left(\mathrm{C}_{\mathrm{n}} \mathrm{E}_{\mathrm{m}}\right)$, have been employed to generate homeotropic anchoring at liquid crystal/water interfaces. The Abbott's group has systematically investigated the influence of the surface coverage and molecular structures of surfactants (e.g., the head group, aliphatic chain length, and aliphatic chain conformation) on the anchoring condition at liquid crystal/water interfaces. Their results have been summarized in a review [35].

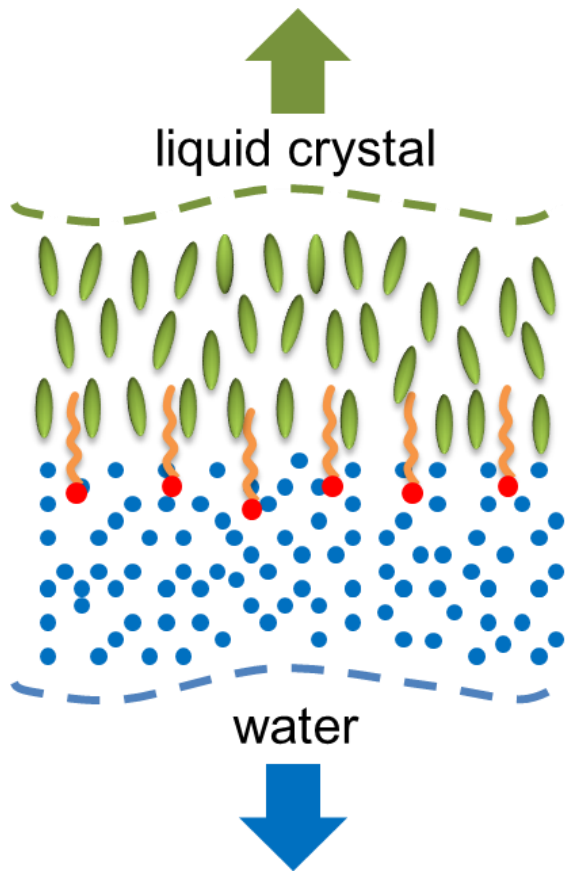

\section{surfactant}

Figure 1.8 Schematic illustration of a liquid crystal/water interface laden with surfactant molecules.

At a first glance, the surfactant laden aqueous interfaces may be considered similar to the SAMs or LB films coated solid substrates (see Section 1.2.2), since in both cases the penetration of aliphatic chains into the adjacent liquid crystal phase is responsible for the effect of 
homeotropic anchoring. However, an essential difference between them is: The self-assembled behavior of the surfactants at the aqueous interfaces is in equilibrium with the bulk solutions of the surfactants, whereas SAMs or LB films at the liquid crystal/solid interfaces are not in equilibrium with a bulk surfactant phase. The equilibrium with the bulk solutions allows for many tunable properties of the surfactant-laden liquid crystal/aqueous interfaces by tuning the bulk properties of the solutions, thus leading to a variety of ordering phenomena of liquid crystals in response to the surfactant adsorption. For instance, the anchoring of 5CB nematic mesogens at an aqueous interface laden with the surfactant SDS was quantitatively investigated with respect to a delicate change of the concentration of SDS in the bulk solution. It was found that the tilt angle of the mesogens evolved continuously from $90^{\circ}$ (planar) to $0^{\circ}$ (homeotropic) upon increasing the surfactant concentration [35]. Moreover, some surfactants may change their structures due to external stimulates such as light, temperature and chemical reactions, which can induce dynamic anchoring transformations [38-40].

Concerning the theoretical aspect, Rey has reported a thermodynamic model for nematic/aqueous interfaces laden with surfactants [69]. Lockwood et al. have connected this model with their experimental observations of anchoring transitions caused by interfacial adsorption of surfactants [35].

\subsection{Surface Ordering at Surfaces and Interfaces of Isotropic Liquids}

The scientific investigation of properties at liquid surfaces or interfaces is a traditional enterprise of ongoing interest [1-5]. Whereas the microscopic forces in the bulk equilibrium phase of an isotropic liquid are uniform, the presence of a surface or an interface breaks the symmetry. The broken symmetry in forces leads to a series of interesting surface-induced thermodynamic properties (such as surface tension and phase transitions) and microscopic structures (for example, molecular orientation and ordering). Although considerable theoretical and experimental progress was made in understanding the properties at surfaces or interfaces of liquids, much resurgence in this scientific enterprise has taken place recently, due to the increasing importance related to industries, as well as the new interests found in the context of complex fluids which include colloids, surfactants, liquid crystals, polymers, etc. [4]. 
(a)

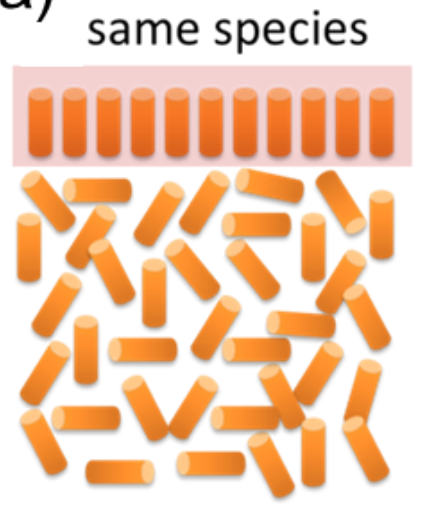

(b) different species

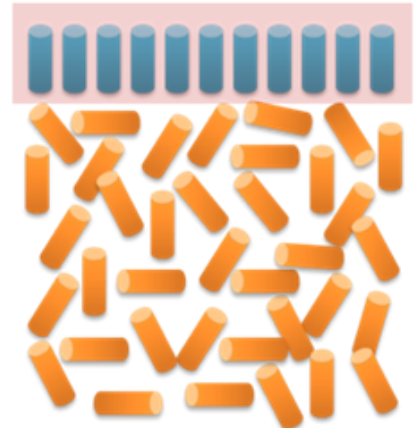

Figure 1.9 Schematic illustration of ordered surface layers formed by (a) the same molecular species of or (b) a different molecular species from the bulk phase.

A topic from the abovementioned vast enterprise is the investigation of the ordering behavior at surfaces and interfaces of liquids: At the surface or interface of many liquids, a highly-ordered surface phase is found in equilibrium with the bulk phase under certain thermodynamic conditions. Depending on the molecular species of the surface phase and the coexistent bulk phase, one can divide the surface ordering of liquids into two classes.

In the first class, as schematically shown in Figure 1.9a, the surface ordered phase is formed by the same molecular species of the coexistent bulk phase. This kind of surface ordering often occurs in the vicinity of a bulk transition going into a more ordered phase. One can find many examples for this behavior such as the surface freezing of n-alkanes and n-alkanols [6-7], the surface layering in liquid metals [8] and ionic liquids [9], and the nematic or smectic surface order in the isotropic phase of thermotropic liquid crystals [10-12]. The various surface ordering behaviors are also understood in terms of wetting [1]. Upon approaching the bulk phase transition, the thickness of the ordered surface layer may diverge into the bulk phase. Such a situation corresponds to complete wetting. The case of complete wetting can be found in the divergent growth of nematic surface layers at the free surfaces of some isotropic liquid crystals which undergo isotropic-nematic phase transitions [70]. The other case is called partial wetting, whereby the surface phase remains a constant thickness as the bulk phase approaches the phase 
transition. The surface freezing of long-chain n-alkanes is a representative phenomenon of partial wetting: A single crystalline monolayer is formed at the free surface of the isotropic liquid at temperatures up to $3{ }^{\circ} \mathrm{C}$ above the bulk freezing temperature [6-7].

(a)

\section{Langmuir film}

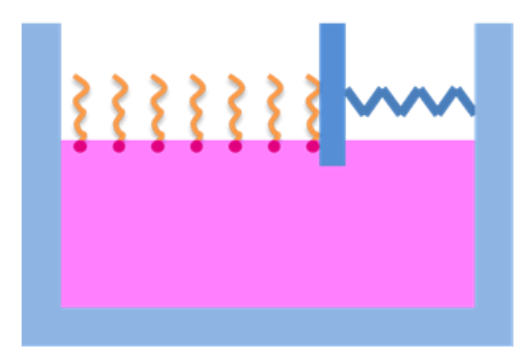

(b)

\section{Gibbs film}

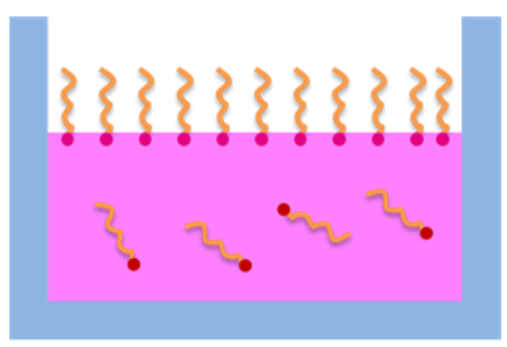

Figure 1.10 Schematic illustrations of (a) a Langmuir film and (b) a Gibbs film.

The second class of surface ordering occurs in the surface layers that possess different constituent molecules from the bulk liquid phase. The surface layers can be formed by insoluble molecules that are spread on the surface of the supporting bulk liquids (Langmuir films) [71] or soluble molecules that are absorbed from the dissolving bulk solutions (Gibbs films). The interfacial molecules are commonly amphiphiles such as surfactants and lipids, and the bulk liquids can be water or other liquids. Figure 10.1a shows a schematic image of a Langmuir film on the surface of water. By controlling the two thermodynamic variables (i.e., temperature and surface pressure) or modifying the molecular architectures of the amphiphiles, a variety of structures and phase transitions of Langmuir films have been discovered. The compression of the molecules can result in structural phase transitions from gaseous states to more condensed states (e.g., 2D crystalline structures). Usually, the surface pressure-area isotherm measurements give the thermodynamic and structural information of the Langmuir films.

With respect to Gibbs films (see Figure 1.10b), they are absorbed amphiphilic layers at the free surfaces of water (or other liquids) [72] or at the interfaces between water and oils [73]. 
Unlike the molecules in Langmuir films, the molecules in Gibbs films are soluble and in equilibrium with the bulk liquid phase. As a result, the mechanical compression by a barrier along the surface that is applied to increase the surface pressure of Langmuir films is not possible in the system of Gibbs films. The formation of 2D crystalline structures in Gibbs films is not as easy as that in Langmuir films. Particularly for the Gibbs films formed at liquid/liquid interfaces, the solvation of the two blocks of the molecules by the two immiscible liquid phases respectively makes the molecules more disordered. Nevertheless, many Gibbs films of surfactants or other amphiphiles have been reported to be able to show solid-like ordered phases at the air interfaces [72] or the liquid/liquid interfaces [74-75]. The formation of a condensed, ordered Gibbs film may rely on strong interactions between the constituent blocks [73]. Thus, amphiphiles with rigid chains such as perfluorinated alkyl chains are prime candidates for the formation of condensed, ordered Gibbs films.

One special kind of amphiphiles, i.e., semifluorinated alkanes, are worthy of mention here, since they can display all the surface ordering phenomena discussed above. Semifluorinated alkane molecules consist of two antagonist moieties: a hydrocarbon block and a fluorocarbon block [76]. Similar to long-chain n-alkanes, this kind of molecules exhibit highly-ordered surface layers at the free surface of their own melts in the vicinity of the freezing temperatures [77]. Despite the absence of a polar head in the molecules, these molecules can also form stable, ordered Langmuir films at the surface of water, which have been demonstrated by many thermodynamic measurements, X-ray diffraction and scattering techniques, and Brewster angle microscopy, etc. [78]. The ordered Langmuir films were transferred by a LB trough to solid substrates for AFM characterizations [79]. It has been revealed that the LB films of semifluorinated alkanes are composed of self-organized surface domains with size about tens of nanometers. In addition, because a perfluorinated chain has a much lower surface tension than a common aliphatic chain, these molecules, when dissolved in hydrocarbon solvents, are able to be absorbed at the free surface, forming Gibbs films [47, 80-81]. The semifluorinated alkanes can serve as "surfactants for oils". More interestingly, the Gibbs films of several semifluorinated alkanes display sharp structural phase transitions from a dilute state at higher temperatures to a dense state at lower temperatures [47]. Thus, such semifluorinated alkanes can be employed to modify the properties of the free surface of liquid crystals. Furthermore, the structural transitions 
of the Gibbs films may have dramatic influences on the surface ordering and anchoring behavior of thermotropic liquid crystals.

\subsection{Surface-Induced Pretransitional Ordering of Liquid Crystals}

Surfaced-induced pretransitional liquid crystalline order that occurs at temperatures above the isotropic-nematic or isotropic-smectic transitions is representative of a class of surface ordering phenomena. Phenomenological [46, 82-84] and microscopic theories [85-86] have treated this class of phenomena from the viewpoint of wetting and adsorption. Thus, the nature of surface anchoring (see Section 1.2), i.e., the interaction between liquid crystals and surfaces, often plays a key role in determining a large variety of predictable wetting behaviors.

In the case of homeotropic anchoring, as the temperature approaches the bulk transition from above, the interface of an isotropic liquid crystal is usually found to be wet by a thin layer of homeotropically aligned nematic or smectic phase. The wetting regimes are strongly correlated to the strength of the homeotropic anchoring. Strong homeotropic anchoring leads to a divergent growth of the thickness of the ordered surface layer, corresponding to complete wetting. Rather week homeotropic anchoring results in partial wetting, whereby the thickness of the wetting layer stays finite. Furthermore, first-order prewetting transitions, characterized by a discontinuous thickness change of the wetting layer (thin to thick), are also possible. Whereas both complete and partial wetting can be found upon approaching isotropic-nematic transitions [70], mainly partial wetting is observed for isotropic-smectic transitions, as evidenced by the growth of a finite number of smectic layers [11-12].

With respect to the situation of planar anchoring, although similar complete or partial wetting with a surface ordered layer of planar alignment was theoretically expected for suitable surface potentials $[82,84]$, yet experimental observation of such surface ordering phenomena turned out to be difficult. Only a few experimental systems have ever shown the growth of a surface phase with negative birefringence [87-88]. Therefore, it is not surprising that the investigation of surface ordering under the condition of homeotropic anchoring has been more fruitful. A brief review on the experimental progress of wetting behavior at interfaces with homeotropic anchoring is presented in the following, whereas the theoretical treatment is discussed in Chapter 2. 
The pioneering work of Miyano, the observation of the solid-wall-induced birefringence in a nematic liquid crystal above the isotropic-nematic transition, has been considered as the first confirmation of the pretransitional surface order [10]. In fact, prior to Miyano's study, the surface tension measurements of the isotropic 5CB by Gannon et al. had already indicated the presence of surface excess order in the vicinity of the isotropic-nematic transition [44]. The surface ordered phase of 5CB was later confirmed by Beaglehole through the Brewster angle ellipsometric study on the surface of the isotropic 5CB [89]. Following these early reports, a number of intensive experimental studies on surface-induced nematic and smectic order have been performed by using techniques such as Brewster angle reflection ellipsometry [41-43, 9094], evanescent wave ellipsometry [95-96], X-ray reflectivity [11-12, 97], differential scanning calorimetry (DSC) [98-99], AFM [100], NMR [101-103], etc. And this behavior has been widely observed at flat interfaces to air [11, 90-94, 97], solid substrates [95-96, 100, 104] and water [4143], as well as in cylindrical nanopores [101-103, 105].

An important parameter for systematic studies of the ordering behavior at an isotropic liquid crystal interface is the strength of the surface field (i.e., the interaction between the liquid crystal and the substrate). A possible approach to varying this parameter is to change the liquid crystal molecules but keeping the substrate unchanged, which can be realized by the use of the homologous series of liquid crystals compounds with tunable alkyl chain length. For example, to achieve the systematic changeover from partial wetting to complete wetting, Chen et al. have employed the homologous series of $\mathrm{nCB}$ ( $\mathrm{n}=5$ to 9 ) on a glass coated with DMOAP [104]. Their results have indicated a partial wetting behavior for $5 \mathrm{CB}$ but complete wetting for $6 \mathrm{CB}$ to $9 \mathrm{CB}$. It has been also shown that the complete wetting character becomes more pronounced with a further increase of the alkyl length of nCB. However, it is obvious that the use of liquid crystal homologues is limited by their availability. In addition, changing the liquid crystal molecules leads to a change of the bulk properties. Thus, a better alternative method to achieve the control over the interfacial interaction without changing the bulk properties of liquid crystals is to modify the surface properties of the substrates. As discussed in Subsection 1.2.2, SAMs of longchain molecules on solid substrates can induce homeotropic anchoring. There are a wide range of molecules capable of forming SAMs on solid substrates. Thus, variation of the molecular geometry of SAMs can be used to control the interfacial interaction between the liquid crystal and the substrate. Crawford et al. have reported systematic studies on the orientational wetting 
behavior of liquid crystals in response to the variation of the length of the surface coupling agent (carboxylic acid). Their results show that above the isotropic-nematic transition temperature, an orientational wetting transition from partial to quasi-complete is observable as the length of the aliphatic acid increases. The surface order parameter increases as the length of the aliphatic acid increases, until a maximum value is reached; thereafter, the surface order parameter decreases as the length of the aliphatic acid increases [101]. Other similar studies concerning the use of semifluorinated SAMs [106] and the variation of $\omega$-functional group in the SAMs [107] have also shown the merits of SAMs in tuning the liquid crystal-substrate interactions.

Although the experimental progress described above demonstrated the crucial importance of control over the surface field in the studies of the surface-induced pretransitional order, the results were far from satisfactory if compared with the theoretical expectations. Theoretical models predicted long time ago a richer variety of surface phase transitions in the vicinity of both the isotropic-nematic [46] and isotropic-smectic bulk transitions [85, 108]. For example, one type of those surface phase transitions are first-order prewetting transitions, which were predicted theoretically but difficult to be observed experimentally [46]. The reason is that observation of such surface phase transitions requires a very subtle range of the strength of the surface field which was not accessible in the earlier experiments. Most of the earlier work was done on solid or air interfaces. However, for liquid crystal/air interfaces, manipulation of the interfacial properties was considered extremely difficult (Chapter 4 will show a feasible approach). As for liquid crystal/solid interfaces, although tuning the properties of coated SAMs monolayers, to some extent, permits a variation of the liquid crystal/substrate interactions in a systematic manner, there are some drawbacks of this means. For one thing, the growth mechanism for SAMs is highly complicated, which involves a stepwise process; for another, control over the properties replies on many experimental parameters such as time of growth, temperature, solvents, and so on [109]. Therefore, the subtle range of the strength of the surface field in which some surface phase transitions occur was difficult to be reached by common experimental processes. It is not surprising that many experimental studies probably aimed to observe more surface phase transitions but finally failed to do so.

Surfactant-laden aqueous interfaces can induce homeotropic anchoring to liquid crystals if the surfactant concentration in the bulk is above a certain minimum value (see Subsection 1.2.2). In 
spite of many merits found in potential biological applications, the most valuable advantage of surfactant-laden liquid crystal interfaces that can be utilized in studying the surface-induced ordering behavior is the feasibility of the exceptional fine control over the strength of the surface field via the variation of the surfactant concentration in the bulk solution. Using Brewster angle reflection ellipsometry, Bahr has studied for the first time the orientational wetting behavior of $8 \mathrm{CB}$ at a surfactant-laden interface to water near the isotropic-nematic bulk transition [41]. By relating the wetting behavior to the Landau model, it has been demonstrated that the surfactant concentration can be used as a tuning parameter for the surface field-the interfacial potential is found to be linear to the surface coverage of the surfactant. Later, Bahr has further applied this important finding in experimentally exploring more surface phase transitions predicted by theoretical models. One successful case has involved the observation of prewetting transitions at nematic liquid crystal/water interfaces. The ellipsometric study of the $9 \mathrm{CB} /$ water interfaces laden with a nonionic surfactant (monoolein) has shown that first-order prewetting transitions can occur near the isotropic-nematic bulk transition if the surfactant concentration is controlled within a certain range; the critical point of the prewetting transition has been observed by increasing the surfactant concentration [43]. Moreover, the tuning of the strength of the surface field at surfactant-laden interfaces is also useful in the study of the surface-induced smectic order above the isotropic-smectic transition. The growth behavior of smectic surface layers at a surfactant-laden interface above the isotropic-smectic transition has been investigated with respect to the change of the surfactant concentration in the bulk phase [42]. The results show that when the surfactant concentration is sufficiently large, the thickness of the smectic surface phase displays a stepwise growth of single smectic layers upon approaching the bulk transition, similar to the behavior observed at the air interfaces. And the temperature of the formation of the first smectic layer decreases as the surfactant concentration decreases, which leads to the merging of the first two or first three smectic single layers to a multiple-layer thickness jump at lower surfactant concentrations.

Thus, these studies have demonstrated that the surfactant concentration can serve as a handle to tune the strength of the surface field at the liquid crystal interfaces so that novel surface phase transitions can be observed experimentally. In this dissertation, other useful methods for the variation of the surface field are explored. In addition, Bahr's work provides an important implication that adsorption of surface-active molecules that is in equilibrium with a bulk phase is 
exceptionally tunable for studies of surface ordering and anchoring behavior of liquid crystals. Inspired by Bahr's work, the possibility of changing the surface ordering and anchoring behavior at the air interface is presented by using a type of highly surface-active molecules (i.e., semifluorinated alkanes) which are capable of segregating to the air interfaces of thermotropic liquid crystals. 


\section{Chapter 2. Experimental Techniques and Theoretical Models}

\subsection{Experimental Techniques}

The optical properties of liquid crystals are critical to their fundamental research and technological applications. Since two optical techniques, i.e., polarized light microscopy and Brewster's angle reflection ellipsometry, are employed in this dissertation to determine the surface anchoring and ordering behavior of liquid crystals at interfaces, it is necessary to start with a brief description of the basic knowledge of optics related to liquid crystal research. We discuss how the two abovementioned optical techniques are operated in detail. Apart from polarizing microscopy and Brewster angle reflection ellipsometry, AFM is employed to characterize the morphology of Gibbs films of a semifluorinated alkane at surfaces of liquid crystal droplets and thus the principle of AFM is also introduced.

\subsubsection{Basic Optics for Liquid Crystal Research}

"Polarized light" is one of the most frequently mentioned terms by researchers of liquid crystals. In general, light can be considered as a transverse electromagnetic wave. Conventionally, the electric field vector $\vec{E}$ is chosen to represent the polarization of light. Natural light or light from other artificial sources is usually made up of waves whose electric field vectors vibrate at all possible angles in the plane perpendicular to the direction of propagation; in this case, the light is called unpolarized light. In order to discuss the polarization state, the oscillating electric field vector $\vec{E}$ can be resolved into two orthogonal components perpendicular to the direction of light propagation. As illustrated in Figure 2.1, the $z$ axis is made the direction

of the light propagation and the two orthogonal components of the electric vector $\left(\vec{E}_{x}\right.$ and $\left.\vec{E}_{y}\right)$ are along the $x$ axis and the $y$ axis, respectively. They can be represented as

and

$$
\begin{gathered}
\vec{E}_{x}(z, t)=\hat{\imath} E_{o x} \cos (k z-\omega t) \\
\vec{E}_{y}(z, t)=\hat{\jmath} E_{o y} \cos (k z-\omega t+\delta),
\end{gathered}
$$


where $\hat{\imath}$ and $\hat{\jmath}$ are the unit vectors, $E_{o x}$ and $E_{o y}$ are the amplitudes, $k$ is the wavenumber, $\omega$ is the angular frequency, $t$ is the time, and $\delta$ is the phase difference between the two waves [110]. If $\delta$ is zero or an integral multiple of $\pm \pi$, then $\vec{E}=\left(\hat{\imath} E_{o x}+\hat{\jmath} E_{o y}\right) \cos (k z-\omega t)$ and the electric field vector vibrates in only one specific plane (see Figure 2.1a). Under such a circumstance, light is said to be linearly polarized (or plane polarized). For linear polarization, it is not necessary that $E_{o x}=E_{o y}$. There is one specific situation in which $E_{o x}=E_{o y}$ and $\delta=-\pi / 2+2 m \pi(\mathrm{m}=0, \pm$ $1, \pm 2 \ldots$ ), then the light is circularly polarized (see Figure 2.1c). In the case of circular polarization, the electric vector rotates with a fixed amplitude $\left(\sqrt{E_{o x}^{2}+E_{o y}^{2}}\right)$ and the projection on the $x-y$ plane traces a circle. The third situation is called elliptical polarization in which $E_{o x} \neq E_{o y}$ and $\vec{E}_{x}$ and $\vec{E}_{y}$ are shifted arbitrarily in phase (see Figure 2.1b). The name of such a polarization state is due to the fact that as the electric vector $\vec{E}$ rotate around the $z$ axis, the projection on the $x-y$ plane sweeps out an ellipse.

In order to transform an unpolarized beam of light into a polarized beam of light, one can use a polarizer. A polarizer is an optical filter that permits the transmission of light of a specific polarization but blocks light of other polarizations. As illustrated in Figure 2.2a, when an unpolarized beam of light passes through the linear polarizer A (the green frame), the light becomes linearly polarized with electric field vectors vibrating vertically. In the characterization of liquid crystal textures, a setup called crossed-polarizers is often needed. Figure $2.2 \mathrm{~b}$ shows the configuration of crossed-polarizers in which the transmission axes of the two polarized are set up $90^{\circ}$ apart (crossed). Under such a circumstance, the polarized light coming out from the polarizer A is extinguished by the polarizer B. The setup of crossed-polarizers is one of indispensable component of a polarizing microscope. If the transmission axes of the two polarizers shown in Figure $2.2 \mathrm{~b}$ are set at an arbitrary angle $\theta$, the light coming from the first polarizer may pass through the second one. The intensity of the light $I$ after two polarizers follows the Malus' Law:

$$
I=I_{1} \cos ^{2} \theta
$$

with $I_{1}$ designating the intensity arriving at the second polarizer [110]. It is obvious that the configuration of crossed-polarizer is a special situation with $\theta=90^{\circ}$ corresponding to $I=0$. 

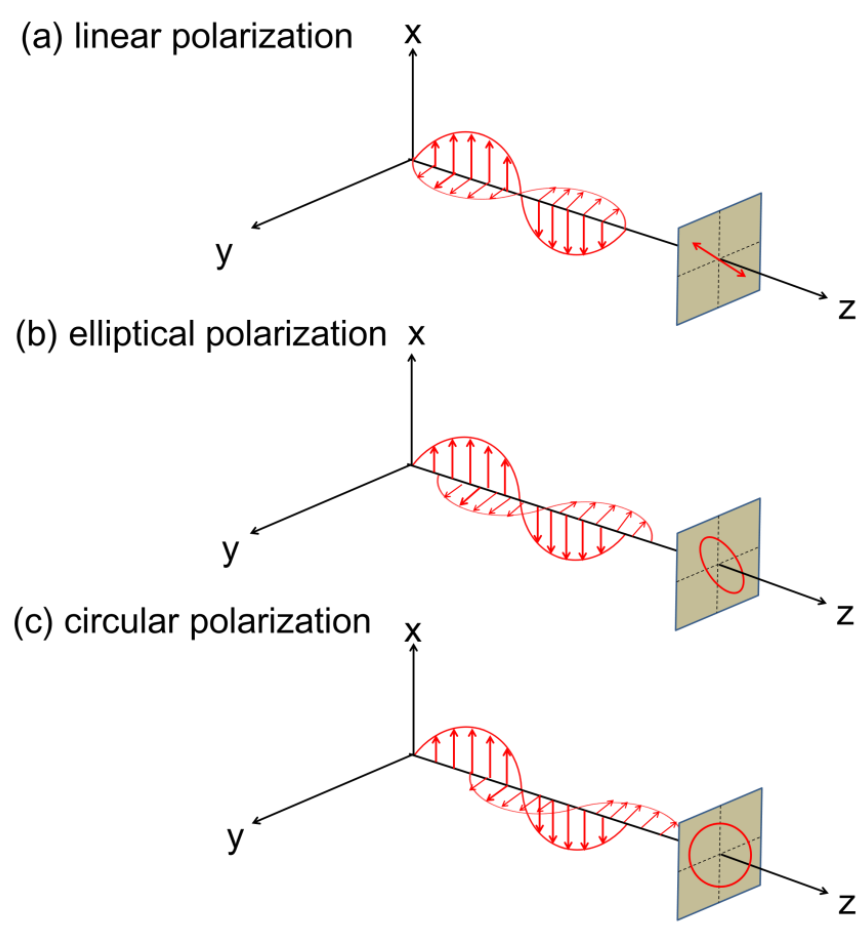

Figure 2.1 Schematic illustrations of polarizations of light: (a) linear polarization, (b) elliptical polarization, and circular polarization. The $z$ axis corresponds to the direction of the light propagation.
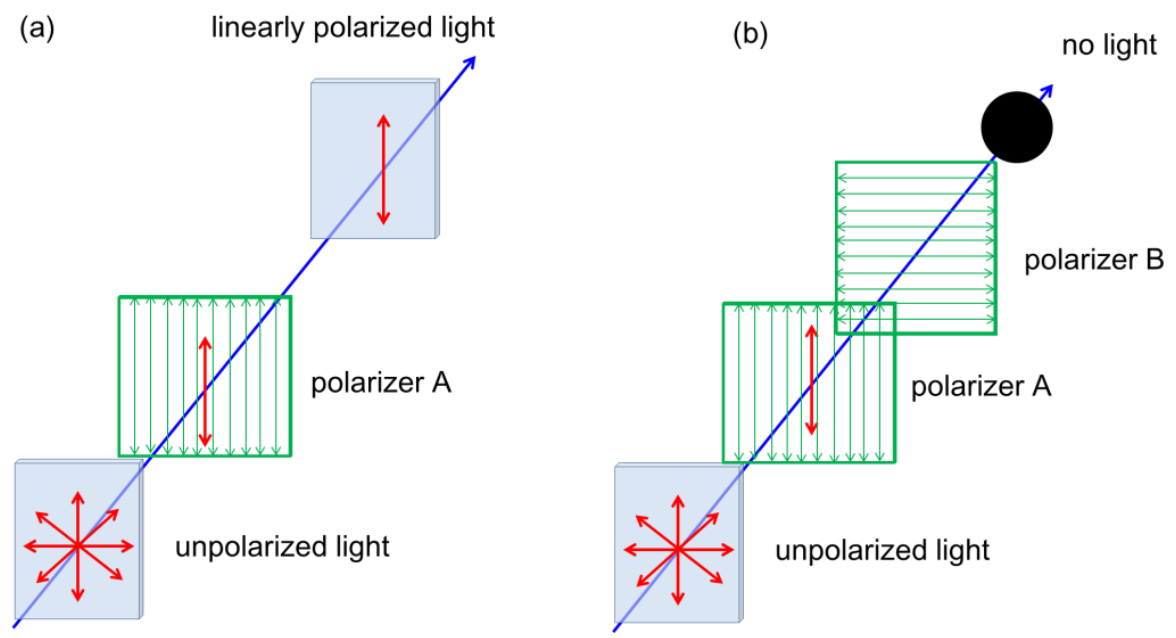

Figure 2.2 Schematic illustrations of an unpolarized beam of light going through (a) a single polarizer and (b) crossed-polarizers. The blue single-ended arrows indicate the direction of light propagation, the red doubleheaded arrows the directions of the oscillation of the electric field vectors, and the green double-headed arrows the transmission axes of the polarizers. 


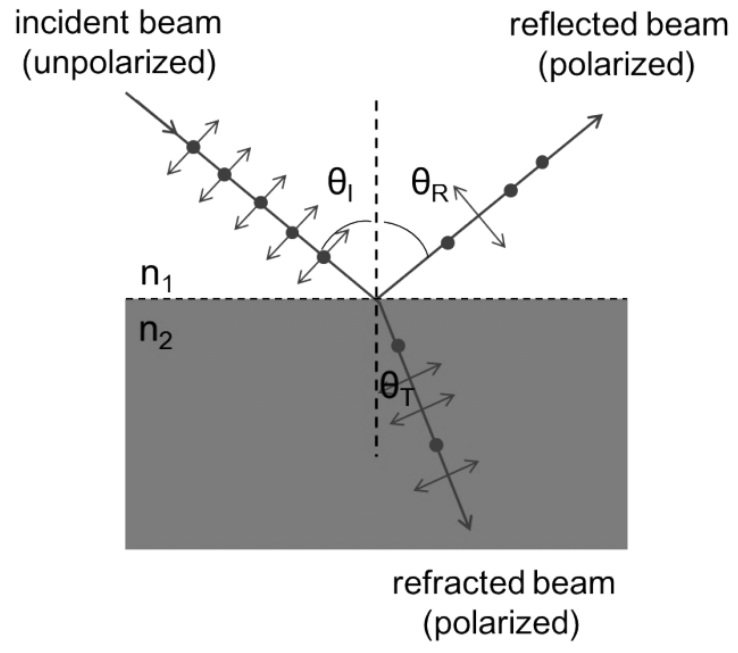

(a)

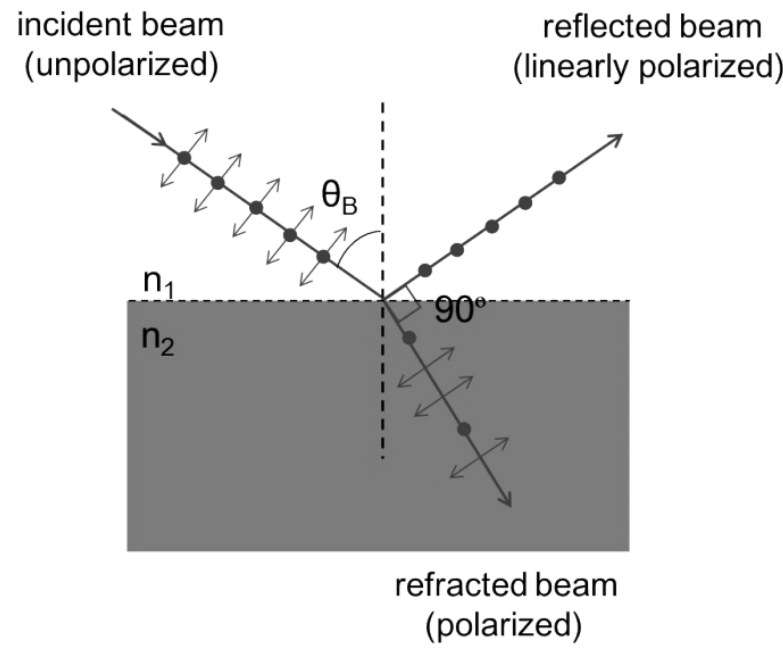

(b)

Figure 2.3 Diagrams of unpolarized light reflected and refracted at an interface between two isotropic media of different refractive indices $\left(n_{1}\right.$ and $\left.n_{2}\right)$. The dots denote the polarization perpendicular to the incident plane $(s$-polarized) and the double-headed arrows the polarization in parallel to the incident plane ( $p$-polarized). The angles of incidence, reflection and refraction are designated by $\theta_{l}, \theta_{R}$, and $\theta_{T}$, respectively. At the Brewster's angle $\theta_{\mathrm{B}}$, the reflected beam is linearly polarized in the direction perpendicular to the plane of incidence.

Polarized light can be also generated by reflection at the flat surface of a dielectric material (see also Subsection 2.2.3 where the principle of ellipsometry is discussed). Figure 2.3a depicts a diagram of an unpolarized beam of light being reflected and refracted on a smooth interface between two isotropic media with refractive indices of $n_{1}$ and $n_{2}$, respectively. The electric field vectors of the incident beam of unpolarized light considered to consist of (arbitrarily many) linearly polarized components which are either $s$ - or $p$-polarized (for $s$-polarized light, the electric field vector vibrates perpendicular to the plane of incidence, for $p$-polarized light, the electric field vector vibrates parallel to the plane of incidence). If the electric field vibrates in parallel to the plane of incidence, the light is considered as $p$-polarized. The second polarization is called s-polarization when the electric field vibrates perpendicular to the plane of incidence. Both of the reflected beam and the refracted (transmitted) beam are polarized. The polarizations and the power of both the reflected beam and the refracted beam depend on the angle of incidence $\theta_{I}$ and the refractive indices of the two media. The fraction of the incident power that is reflected from the interface is given by the reflectance $R$ which can be considered in the cases of 
$s$-polarization $\left(R_{s}\right)$ and $p$-polarization $\left(R_{p}\right)$, separately. The calculation of the reflectance $R_{s}$ and $R_{p}$ is according to the Fresnel's equations.

For the $s$-polarized light,

$$
R_{S}=\left|\frac{n_{1} \cos \theta_{I}-n_{2} \cos \theta_{T}}{n_{1} \cos \theta_{I}+n_{2} \cos \theta_{T}}\right|^{2}=\left|\frac{n_{1} \cos \theta_{I}-n_{2} \sqrt{1-\left(\frac{n_{1}}{n_{2}} \sin \theta_{I}\right)^{2}}}{n_{1} \cos \theta_{I}+n_{2} \sqrt{1-\left(\frac{n_{1}}{n_{2}} \sin \theta_{I}\right)^{2}}}\right|^{2},
$$

where the angle of refraction $\theta_{T}$ in the first form is estimated by using Snell's law and trigonometric identities, hence giving the second form.

For the $p$-polarized light,

$$
R_{p}=\left|\frac{n_{1} \cos \theta_{T}-n_{2} \cos \theta_{I}}{n_{1} \cos \theta_{T}+n_{2} \cos \theta_{I}}\right|^{2}=\left|\frac{n_{1} \sqrt{1-\left(\frac{n_{1}}{n_{2}} \sin \theta_{I}\right)^{2}}-n_{2} \cos \theta_{I}}{n_{1} \sqrt{1-\left(\frac{n_{1}}{n_{2}} \sin \theta_{I}\right)^{2}}+n_{2} \cos \theta_{I}}\right|^{2}
$$

Or the first term of Equation (2.5) can be also transformed into

$$
R_{p}=\left|\frac{\tan \left(\theta_{I}-\theta_{T}\right)}{\tan \left(\theta_{I}+\theta_{T}\right)}\right|^{2} .
$$

It is obvious that if $\theta_{I}+\theta_{T}=90^{\circ}, R_{p}$ is equal to zero; under this condition the $p$-polarized light vanishes in the reflected beam. The use of Snell's law gives an expression for the Brewster angle:

$$
\theta_{B}=\tan ^{-1} \frac{n_{2}}{n_{1}}
$$

As illustrated in Figure 2.3b, the Brewster's angle is the angle at which no p-polarized light is reflected. The physical origin of Brewster's angle can be qualitatively understood by considering the response of electric dipoles to the $p$-polarized light. When the light is incident on the interface, electric dipoles in the material are induced by absorbing light. The induced electric dipoles oscillate and produce the transmitted (refracted) light and the reflected light. However, electric dipoles do not emit any energy in the direction along which they oscillate. The $p$ polarized component vibrates perpendicular to the transmitted beam in the plane of incidence. 
Thus, if the reflected beam is perpendicular to the transmitted beam, the p-polarized light cannot be created in the reflected beam. It should be noted that the expressions from (2.5) to (2.7) are restricted to the situation when a beam of light is incident at the interface between two isotropic media. If the light is incident at the surface of an anisotropic medium, the calculation of the Brewster's angle is more complicated. Consider the free surface of water as an example. In this case, $n_{1}=1$ and $n_{2}=1.333$, then $\theta_{B}=53.1^{\circ}$ as calculated by using Equation (2.7).

(a)

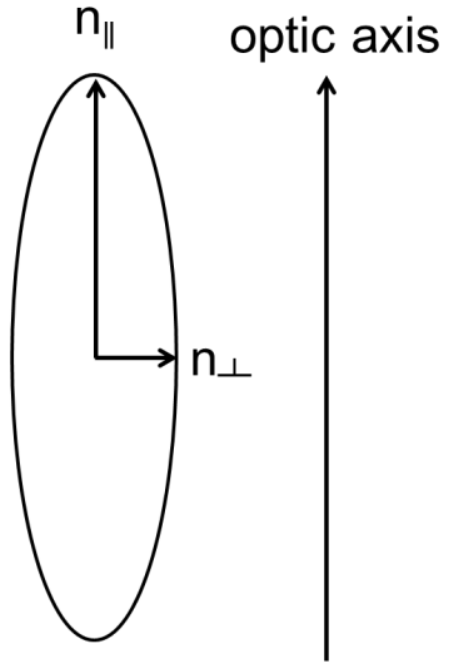

(b)

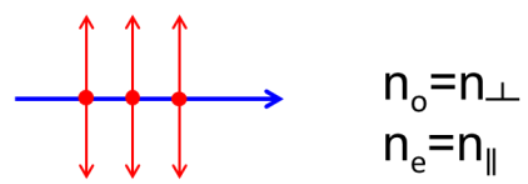

(c)

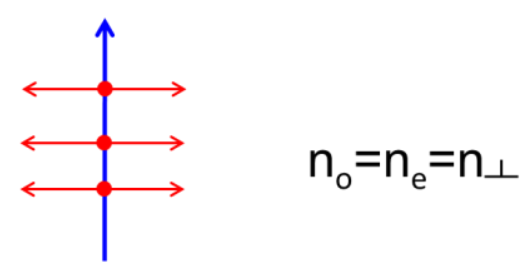

(d)

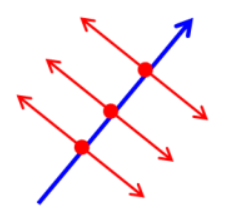

$$
\begin{aligned}
& \mathrm{n}_{\mathrm{o}}=\mathrm{n}_{\perp} \\
& \mathrm{n}_{\mathrm{e}} \neq \mathrm{n}_{\|}
\end{aligned}
$$

Figure 2.4 Schematic illustrations of (a) the principal refractive indices $n_{\|}$and $n_{\perp}$ of a uniaxial material and the ordinary and extraordinary refractive indices $n_{o}$ and $n_{e}$ in three different situations depending on the direction of the light propagation: (b) perpendicular to the optic axis, (c) parallel to the optic axis and (d) with an angle to the optical axis. The blue arrows indicate the light propagation and the red dots and double headed arrows the polarizations.

The concept of polarization is essential to liquid crystal research, because liquid crystals belong to birefringent materials which may change the polarization of light passing through them. Birefringence refers to the optical property of a material that displays two different refractive indices depending on the polarization and propagation direction of light. If a ray of unpolarized light passes through a birefringent material, it may be decomposed into two separate polarized rays. The polarization direction of the two rays is perpendicular to each other. One ray, termed as the ordinary ray, obeys the law of refraction; this ray propagates at the same speed in all directions through the material, hence possessing the constant refractive index (denoted as $n_{o}$ ). 
However, the other ray behaves in an unusual fashion with the refractive index (denoted as $n_{e}$ ) depending on the direction of its travel. This ray is thus called the extraordinary ray. For some optically anisotropic materials, there is a single direction through which the travel of light turns out to be optically the same as that through an isotropic medium. Such a direction is defined as the optical axis. Both of the nematic and the smectic- $A$ liquid crystals are uniaxial and the optic axis is coincident with the director of the liquid crystals.

The two refractive indices $n_{o}$ and $n_{e}$ are related to the principal refractive indices $n_{\Downarrow}$ and $n \perp$ in the directions parallel and perpendicular to the optic axis, respectively (see Figure 2.4a). For the simplicity, the optic axis shown in Figure $2.4 \mathrm{a}$ is deliberately made parallel to the page. In any cases shown in Figure 2.4c-d, we always have

$$
n_{o}=n_{\perp}
$$

because the electric field of the light that is polarized perpendicular to page will always "see" the same refractive index perpendicular to the optic axis $n_{\perp}$. The extraordinary index $n_{e}$ varies with respect to the propagation direction. If the light propagates in the direction perpendicular to the optic axis, we have $n_{e}=n_{\|}$(see Figure 2.4b). If the light propagates in the direction parallel to the optic axis (see Figure 2.4c), we arrive at a special situation in which $n_{e}=n_{o}=n_{\perp}$ and the medium acts like isotropic. If the direction of the light propagation forms an arbitrary angle $\phi$ with the optic axis (see Figure 2.4d), the extraordinary index $n_{e}$ should be calculated by

$$
n_{e}=\frac{n_{\|} n_{\perp}}{\sqrt{n_{\|}^{2} \cos ^{2} \phi+n_{\perp}^{2} \sin ^{2} \phi}} .
$$

We can quantify the birefringence by

$$
\Delta n=n_{e}-n_{o} .
$$

The measurement of the birefringence of liquid crystals is useful for the determination of their orientational order. The observation of the surface induced pretransitional birefringence at the isotropic phase of a nematic liquid crystal has provided strong evidence for the presence of the surface induced pretransitional order in liquid crystals [10]. The property of birefringence is also 
critical to the application of polarizing microscopy and Brewster angle reflection ellipsometry in the research presented in this dissertation.

\subsubsection{Polarized light Microscopy}

Polarized light microscopy is an indispensable tool for studying liquid crystal textures [51]. In Chapter 4, it is employed to characterize the textures of liquid crystal films which can give information about the surface anchoring. Figure 2.5 shows a photo of the polarized light microscope (Nikon Eclipse VL100) used in the study of this dissertation and a schematic image for the simplified configuration of the transmission mode of the microscope. It is known from Subsection 2.1.1 that a beam of unpolarized light passing through the crossed-polarizers must be extinguished. However, a birefringent material which is capable of changing the polarization of light, if placed in between the crossed-polarizers, can allow light to pass through the second polarizer. As shown in Figure 2.5b, the liquid crystal sample is usually placed in between the two crossed-polarizers (the one close to the eyepiece is often called the analyzer) under the transmission mode of the microscope. Because of the birefringent property of the liquid crystal, the linearly polarized light coming out from the first polarizer can be converted to elliptically polarized light depending on the orientation of the liquid crystal director. Usually, an elliptically polarized wave can be resolved into two linearly polarized components (see Subsection 2.1.1), allowing one component to pass through the analyzer. The intensity of the transmitted light from the analyzer $I$ can be quantified by

$$
I=I_{0} \sin ^{2} 2 \varphi \sin ^{2}\left(\frac{\pi}{\lambda} d \Delta n\right)
$$

where $I_{0}$ is the intensity after the polarizer, $\varphi$ the azimuthal angle of the director $\vec{n}$ (see also Figure 2.6a), $\lambda$ the vacuum wavelength, $d$ the distance traveled in the sample, and $\Delta n$ the birefringence of the sample (determined by Equations 2.8-2.10) [51]. The optic textures and the surface anchoring behavior of a liquid crystal film can be related by Equations 2.8-2.11.

For instance, the surface anchoring of a liquid crystal film sandwiched by two cover slips in the absence of any external field (see Figure 2.6b) can be detected by a polarized light microscope. If both of the cover slips induce homeotropic anchoring to the liquid crystal, the director $\vec{n}$ is oriented parallel to the direction of the light propagation $(\phi=0)$; in this case $\Delta n=$ 
(a)
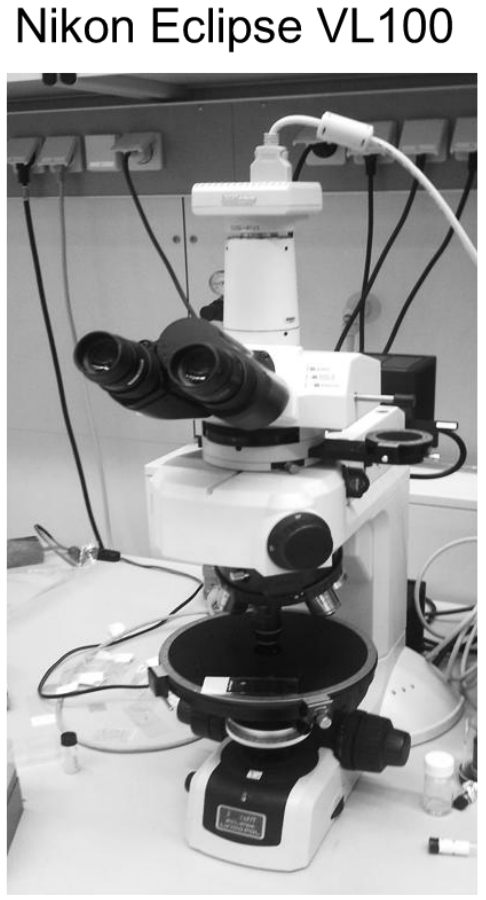

(b)

a simplified configuration

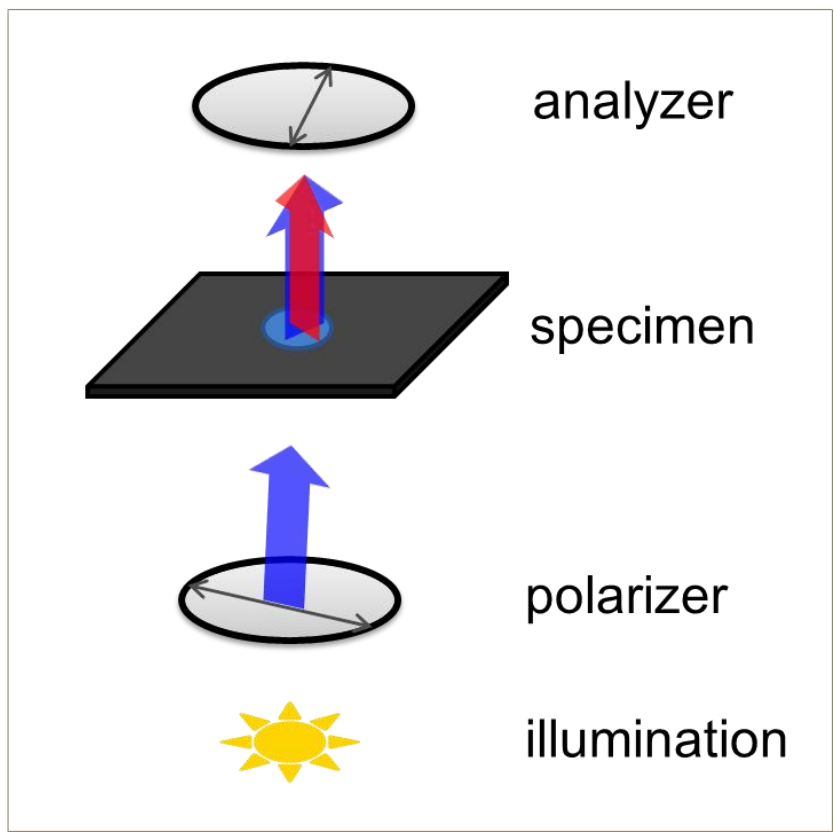

Figure 2.5 (a) Photo of the polarized light microscope Nikon Eclipse VL100 and (b) schematic illustration of a simplified configuration of the microscope.

(a)

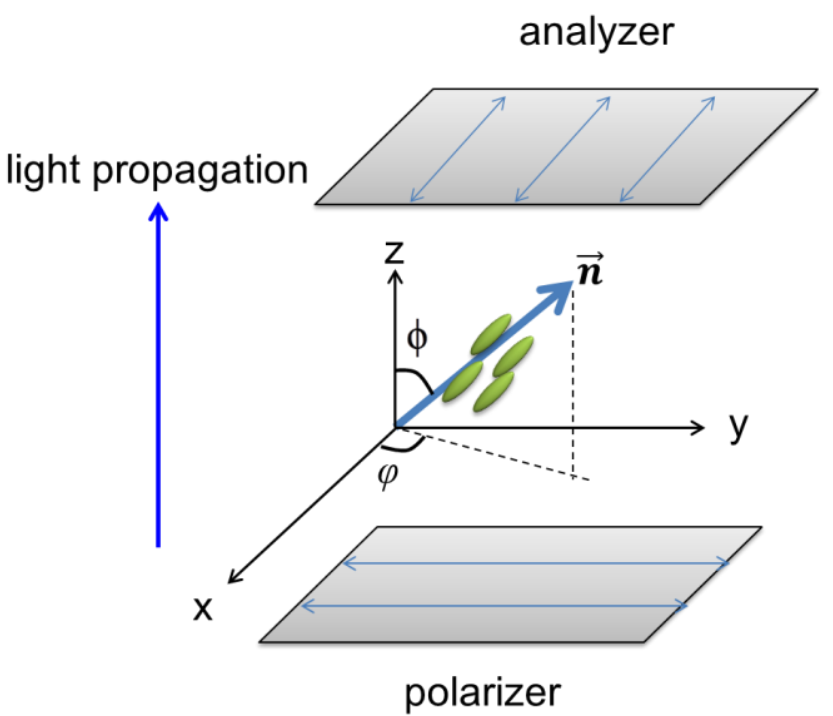

(b)

liquid crystal sample

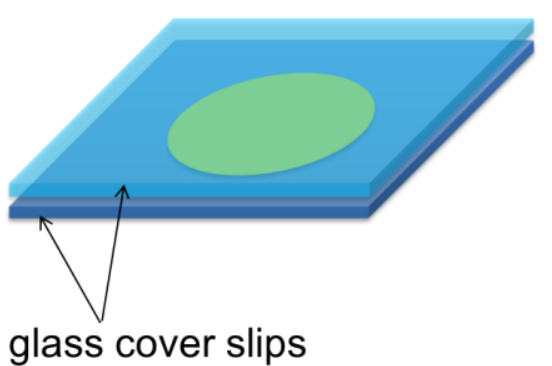

Figure 2.6 Schematic illustration of a liquid crystal sample with a well-oriented director placed between the crossed-polarizers. The direction of the light propagation corresponds to the $z$ axis. The angles shown in (a) are defined by Equations 2.6-2.9 in the text. The liquid crystal sample is sandwiched by two cover slips of which the anchoring properties can be varied. 
0 and $I=0$, so the liquid crystal sample appears dark under the polarized light microscope. If the liquid crystal is uniformly aligned in the plane parallel to the cover slips $\left(\phi=90^{\circ}\right), \Delta n$ is equal to $\left(n_{\|}-n_{\perp}\right)$ and the intensity $I$ is determined by $\varphi$ which can be varied by rotating the liquid crystal sample. When the director $\vec{n}$ is aligned along the transmission axis of the polarizer or the analyzer, we have $\sin ^{2} 2 \varphi=0$ and $I=0$, and the sample looks dark. The intensity reaches the maximum when $\varphi=45^{\circ}$. If both of the cover slips induce random planar anchoring to the liquid crystal, some regions may appear to be dark but others bright since the azimuthal angle of the director $\vec{n}$ is not defined. This situation can be evidenced by the Schlieren texture found in nematic liquid crystals (see also Figure 1.6a in Chapter 1). If the two interfaces induce different anchoring conditions, for instance, homeotropic and random planar anchoring, respectively, a smectic- $A$ film can form focal conic textures (see also Figure $1.6 \mathrm{~b}$ in Chapter 1 ).

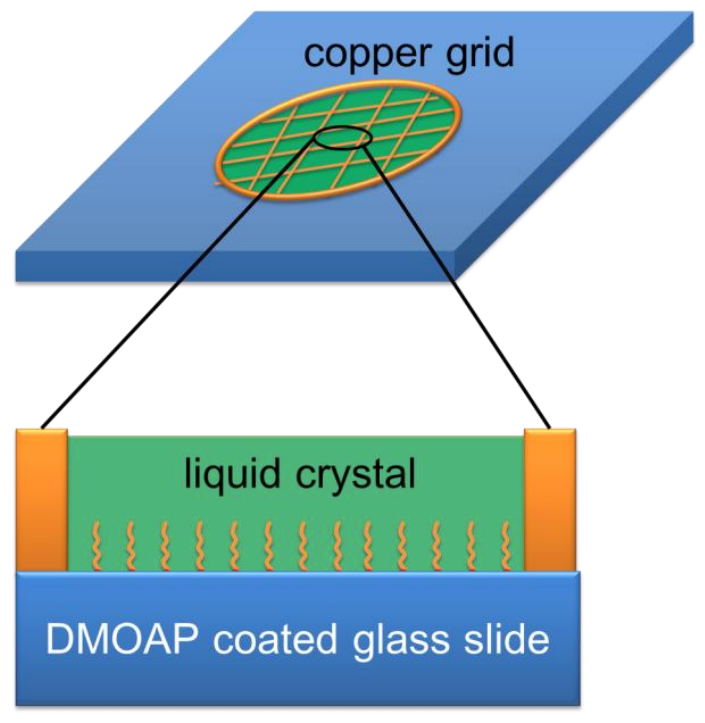

Figure 2.7 Schematic illustration of a thin liquid crystal film with a uniform thickness of $20 \mu \mathrm{m}$ prepared within a TEM grid on a DMOAP-coated substrate.

In order to investigate the anchoring behavior of liquid crystals at liquid crystal/air interfaces (see Chapter 4), an experimental system which is designed by Abbott et al. for preparing liquid crystal films with a uniform thickness is employed here [32]. In this system, copper grids that are actually designed for transmission electron microscopy (TEM) are used as optical cells. The 
TEM grids possessing square holes with a lateral width of $420 \mu \mathrm{m}$ and a thickness of $20 \mu \mathrm{m}$ are purchased from SPI Supplies and cleaned by organic solvents before use. In a typical experiment, a TEM grid is placed onto a glass slide which is coated by DMOAP for inducing strong homeotropic anchoring at the liquid crystal/glass interface (DMOAP is purchased from ABCR, Germany and the detailed coating procedure can be found in [61]). A tiny drop of the liquid crystal in the isotropic phase is deposited onto the grid, which often leads to the formation of a spherical cap on top of the grid. Then, a capillary tube is used to suck the excess liquid crystal. This procedure allows for the formation of a thin liquid crystal film with uniform thickness, as schematically illustrated in Figure 2.7. Because of the pinning by the copper frames of the TEM grid, this type of films is rather stable and can be even immersed into an immiscible liquid such as an aqueous solution [32]. Since one surface of the film is homeotropically anchored by the DMOAP-coated substrate, the anchoring behavior from the other surface can be known by the observation of the optical texture under a polarized light microscope. In Chapter 4, the advantage of this system will be shown in studying the anchoring behavior at liquid crystal/air interfaces laden with semifluorinated molecules.

\subsubsection{Brewster's Angle Reflection Ellipsometry}

Ellipsometry is an old but useful optical technique used to analyze the dielectric properties of thin films. Because it is non-perturbing and nondestructive to specimens, this technique has been applied in industries such as microelectronics, optical coating, and polymer film technologies, as well as fundamental research including surface chemistry and semiconductor physics. Ellipsometry measures the relative change of the polarization after the reflection from the samples, which is highly precise and reproducible.

In the experimental study presented in this dissertation, interfaces of isotropic liquid crystals are probed by ellipsometry which provides information about the thickness and orientation of the interfacial liquid crystal wetting layers. As the liquid crystals under investigation are interfaced to a fluid or the air, two experimental setups are employed here, as schematically illustrated in Figure 2.8. For the study of surfactant-laden liquid crystal/fluid interfaces (see Chapter 3), the setup used here is the same one as the previous studies [41-43]. The liquid crystal sample is placed into a Teflon tube (diameter $7 \mathrm{~mm}$ ) which dips into a reservoir containing the water- 
glycerol phase (see figure 2.8a). The thickness of the liquid crystal film amounts to several mm, so that the liquid crystal/vapor interface is well separated from the liquid crystal/fluid Interface.

(a)

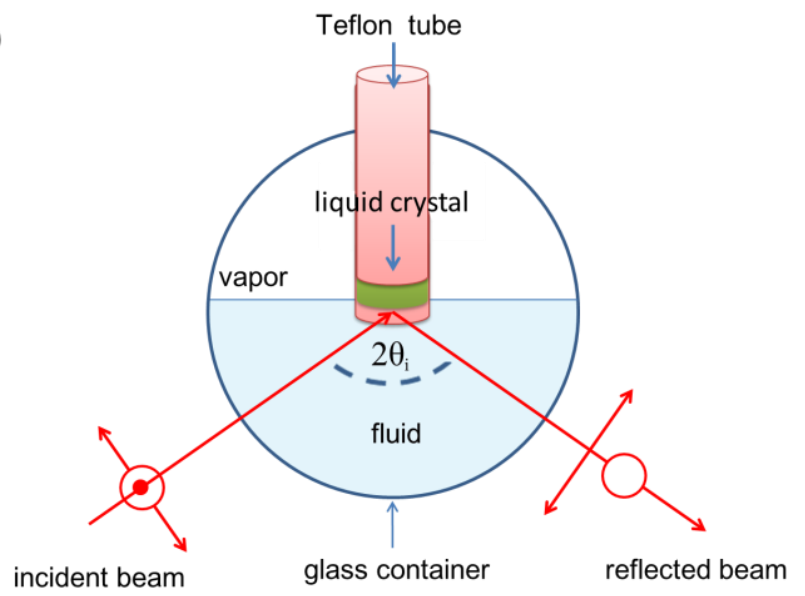

(b)

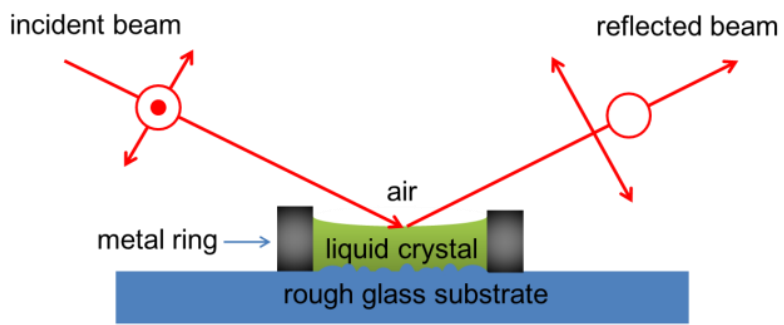

Figure 2.8 Schematic illustrations for the setups for ellipsometric measurements on (a) liquid crystal/fluid interfaces and (b) liquid crystal air interfaces.

Since the water-glycerol phase does not intrude between the organic liquid crystal and the Teflon surface, it is possible to tune the curvature of the liquid crystal/fluid interface by adjusting the immersion depth of the Teflon tube, and a plane interface, suitable for ellipsometric measurements, can be prepared. The liquid crystal/fluid interface is located in the center of a spherical glass container which is placed in a copper oven allowing for optical access of the incident and reflected laser beam of the ellipsometer. For the ellipsometric measurements on liquid crystal/air interfaces as presented in Chapter 4, the schematic setup is shown in Figure 2.8b. A metal ring is glued to a glass substrate to form a cell for containing liquid crystal samples. The surface of glass substrate must be rough in order to eliminate the possible reflection from the substrate surface. An appropriated amount of the liquid crystal sample is filled into the cell, thus 
forming an approximately $1 \mathrm{~mm}$ thick film on the rough glass substrate. The whole sample is placed into a brass oven which allows for optical access of the incident and reflected laser beam of the ellipsometer.

As discussed in Subsection 2.1.1 (see also Figure 2.3), when light is incident at a surface, mostly the polarization state of the reflected beam is different from the incident beam. The name of ellipsometry comes from the fact that linearly polarized light, while incident at a surface, becomes elliptically polarized in the reflected light. In general, there are two angles found in ellipsometric data, i.e., $\Delta$ and $\Psi$, which are defined by

$$
\tan \Psi e^{i \Delta}=r_{p} / r_{s}=\left|r_{p} / r_{s}\right| e^{i\left(\delta_{p}-\delta_{s}\right)},
$$

where $r_{p}$ and $r_{s}$ are the complex Fresnel reflection coefficients of the $p$-polarized and s-polarized light, $\delta_{p}$ and $\delta_{s}$ denote the phase shift of the $p$-polarized and $s$-polarized components in the reflected light. Usually, $\tan \Psi$ and $\Delta$ are called ellipsometry parameters. Let us recall the reflection from an ideal interface between two isotropic media with indices of refractive $n_{l}$ and $n_{2}$ [see also Figure 2.3 and Equations (2.5) to (2.7)]. In Subsection 2.1.1 the reflectances $R_{p}$ and $R_{s}$ have already been given and here we have the relationships $r_{p}=e^{i \delta_{p}} \sqrt{R_{p}}$ and $r_{s}=e^{i \delta_{s}} \sqrt{R_{s}}$. Then, $\tan \Psi$ and $\Delta$ are the ratio of the amplitudes and the phase difference of the $p$-polarized and $s$-polarized components in the reflected light, respectively. Moreover, it is known that when light is incident at the Brewster's angle, $R_{p}=0$; then at the Brewster's angle $\tan \Psi=0$. Let us consider again the free surface of pure water (the refractive index $n_{\text {water }}$ is 1.333) as an example and first suppose that the interface is ideally sharp. The incident beam is a linearly polarized light with $\tan \Psi=1$ and $\Delta=0$. If the calculated values of $\tan \Psi$ and $\Delta$ of the reflected beam are plotted as functions of the incident angle $\theta_{I}, \tan \Psi$ is equal to zero and the value of $\Delta$ jumps by $180^{\circ}$ at the Brewster's angle $\left(53.3^{\circ}\right)$. However, the real interfaces are not really sharp: for example, at the free surface of water, because the water molecules are not strictly confined in the water phase, there is no such a really sharp boundary for the water phase and the air phase. Thus, the surface of water is not a step-function of dielectric profile as presumed an ideal interface. As shown in Figure 2.9, the measured value of $\tan \Psi$ is no longer equal to zero and $\Delta$ is continuous at the Brewster's angle, which is defined as the angle of incidence when $\Delta=90^{\circ}$. 

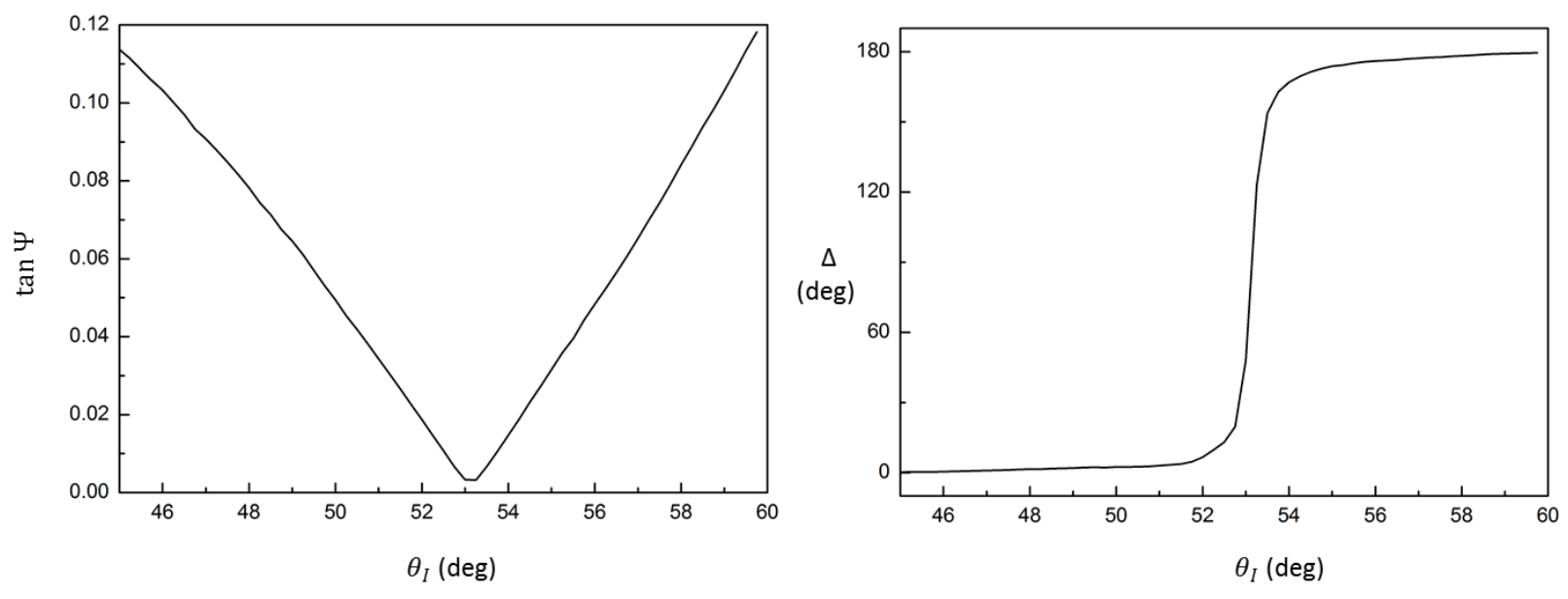

Figure 2.9 Measured $\tan \Psi$ and $\Delta$ at the free surface of water.

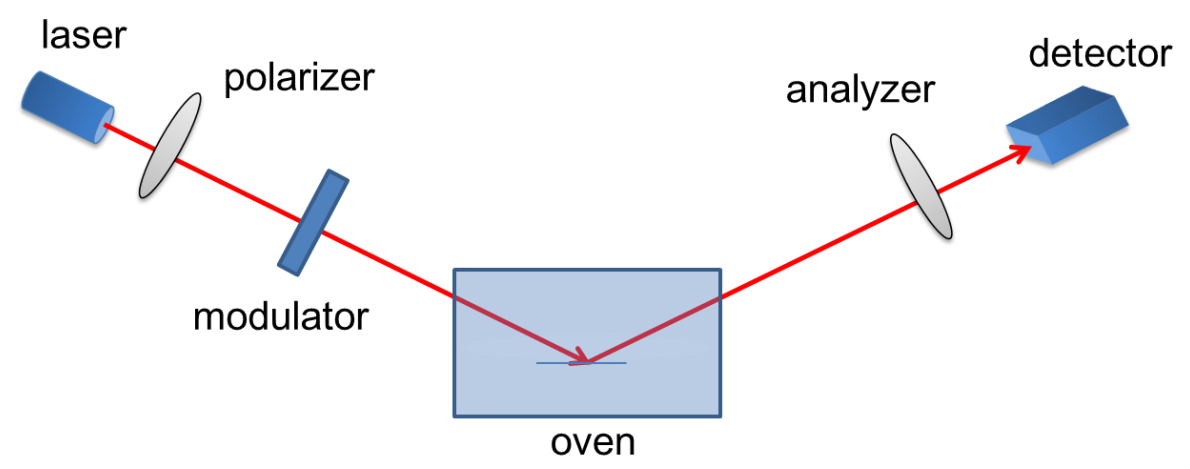

Figure 2.10 Schematic illustration of a phase-modulated ellipsometer.

The value $\tan \Psi$ at the Brewster's angle, often designated as the ellipticity coefficient $\bar{\rho}$, is most sensitive to the presence of an interfacial layer which differs in its optical properties from the two bulk media. For the case of a thin nematic or smectic film on an isotropic liquid crystal bulk phase, possessing typical refractive index values of thermotropic liquid crystal compounds, the magnitude of $\bar{\rho}$ can be considered as a linear measure of the thickness of a nematic or smectic film as long as the film thickness is below $\approx 50 \mathrm{~nm}$.

In the experimental study presented here, a phase modulated ellipsometer is employed to determine the values of $\tan \Psi$ and $\Delta$ at liquid crystal interface. The principles of operation of a 
phase modulated ellipsometer can be found in [111] . Figure 2.10 shows a schematic illustration of the setup of a home-made phase modulated ellipsometer which is the same one used in [70]. The laser beam with a wavelength of $633 \mathrm{~nm}$ passes through the linear polarizer, the polarization axis of which is set at an azimuthal angle of $45^{\circ}$ with respect to the plane of incidence. Then, the phase difference $\delta_{\mathrm{pm}}$ between the $p$ - and $s$-polarized components of the laser beam is modulated by a photoelastic modulator as

$$
\delta_{\mathrm{pm}}=\delta_{0} \sin (2 \pi v t) \text {, }
$$

with $\delta_{0}=138^{\circ}$ and $v=50 \mathrm{kHz}$. The laser beam is incident at the interface of a liquid crystal sample and the reflected light passes through the analyzer which is fixed at an azimuthal angle of $-45^{\circ}$ with respect to the plane of incidence. The laser beam finally reaches a detector which measures the intensity $I$ behind the analyzer. The light intensity $I$ at the detector is a function of the phase difference $\delta_{\mathrm{pm}}$ and of the ellipsometric angles $\Psi$ and $\Delta$ of the interface of the sample. The procedure of determining $\Psi$ and $\Delta$ from the measured $I$ is described in [70]. During a measurement run, the values of $\tan \Psi$ and $\Delta$ are determined and the incident angle $\theta_{I}$ is adjusted so that $\Delta=90^{\circ} \pm 5 \%$. Under this condition, $\theta_{I}$ is a good approximation of the Brewster's angle $\theta_{B}$ and the value of $\tan \Psi$ corresponds to $\bar{\rho}$.

The measured values of $\Delta$ and $\bar{\rho}$ cannot directly give useful information of the refractive index or thickness of the interfacial layer. In order to retrieve such information, one has to consider appropriate models to link the measured ellipsometric parameters to those of interest. The technique of ellipsometry relies on the chosen models in which the physical quantities of interest are not measured directly but only estimated in the framework of the model. Rather commonly, ellipsometric data have been analyzed in terms of Drude's approximate equation:

$$
\bar{\rho}=\frac{\pi \sqrt{n_{a}^{2}-n_{b}^{2}}}{\lambda\left(n_{a}^{2}-n_{b}^{2}\right)} \int_{-\infty}^{+\infty} \frac{\left(n_{a}^{2}-n^{2}\right)\left(n^{2}-n_{b}^{2}\right)}{n^{2}} \mathrm{~d} z,
$$

with $\lambda$ being the vacuum wavelength of the reflected light, $n$ the refractive index at a distance $z$ from the interface, $n_{a}$ and $n_{b}$ the refractive indices of the two media [112]. Instead of utilizing the approximate Drude's equation, the ellipsometric data obtained in this dissertation are 
approximated by a multilayer system developed by Crook [113] with several hundred birefringent layers possessing constant refractive indices.

\subsubsection{Atomic Force Microscopy}

In the research described in Chapter 5, atomic force microscopy (AFM) is employed to visualize the ordered structures formed in Gibbs films of semifluorinated alkane molecules at the surfaces of liquid crystals. AFM belongs to the family of scanning probe microscopy which utilizes a physical probe to scan the surface of a specimen. The technique of AFM is capable of characterizing and manipulating matter of nanoscale at surfaces in environments of vacuum, air, and even liquid. Figure 2.11a simply illustrates the basic principle of AFM. The AFM contains a sharp tip (less than $10 \mathrm{~nm}$ in diameter at the apex) that is responsible for the interactions with the surface. This sharp tip is attached to the free end of a cantilever (typically 100-500 $\mu \mathrm{m}$ long). During the measurement, the tip is approached to the sample surface, resulting in the generation of forces between the tip and the sample surface. The forces cause the cantilever to bend or deflect. A laser beam is incident at the cantilever and the reflected beam is detected by a position-sensitive photodiode. The forces may vary at different locations during the scanning, which cause different deflections of the cantilever and hence different positions of the laser spot at the photodiode. The photodiode measures the deflections of the cantilever which can be transformed by the computer into a map of surface topography. There are three primary imaging modes for a typical AFM: tapping mode, contact mode and non-contact mode. These three modes are operated on the basis of the nature of forces (attractive or repulsive forces) involved in the tip-sample interactions.

Tapping mode is employed here for imaging the self-organized structures of a semifluorinated alkane on highly soft liquid crystal surfaces (see Chapter 5). In tapping mode, the cantilever oscillates at a frequency close to its resonance and the tip lightly taps the surface (see Figure 2.11b) [114]. The change of cantilever's oscillation amplitude with the sample surface is monitored. The cantilever oscillates at a free oscillation amplitude when the tip is far away from the surface. As the tip gets closer to the surface of the sample, the oscillation amplitude is damped. If the tip encounters a bump at the surface, there is less room for the cantilever to oscillate. In contrary, a depression gives more room to the cantilever to oscillate and hence the amplitude is increased. The detector measures the oscillation amplitude of the tip and inputs the 
feedback signal required to maintain a constant amplitude to the controller. Then, the constant amplitude can be maintained by adjusting the separation between the tip and the sample. Therefore, the topography of the surface is obtained by recording the forces of the intermittent tip-sample contacts. Compared to contact mode, tapping mode reduces the possible damages done to the surfaces of the specimens, particularly those made of soft materials. Moreover, this technique is more efficient than the non-contact mode, because the non-contact mode uses much smaller oscillating amplitude and the non-contact tip is easily stuck in the thin layer of liquid on most sample surfaces in ambient environments.

(a)

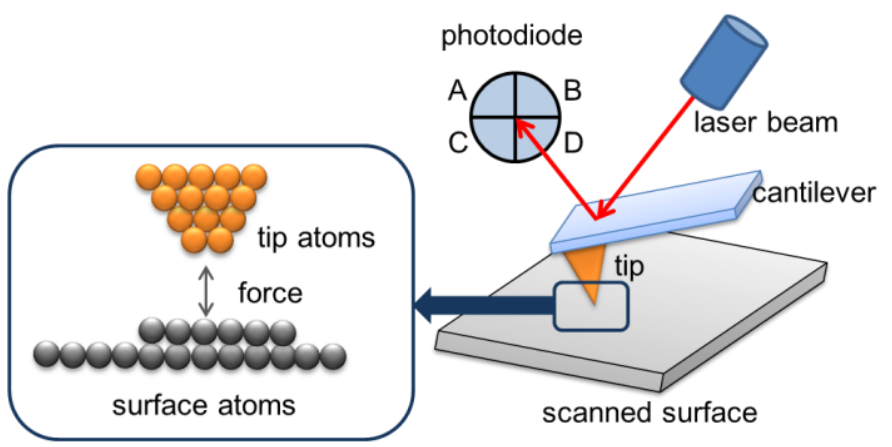

(b) tapping-mode AFM
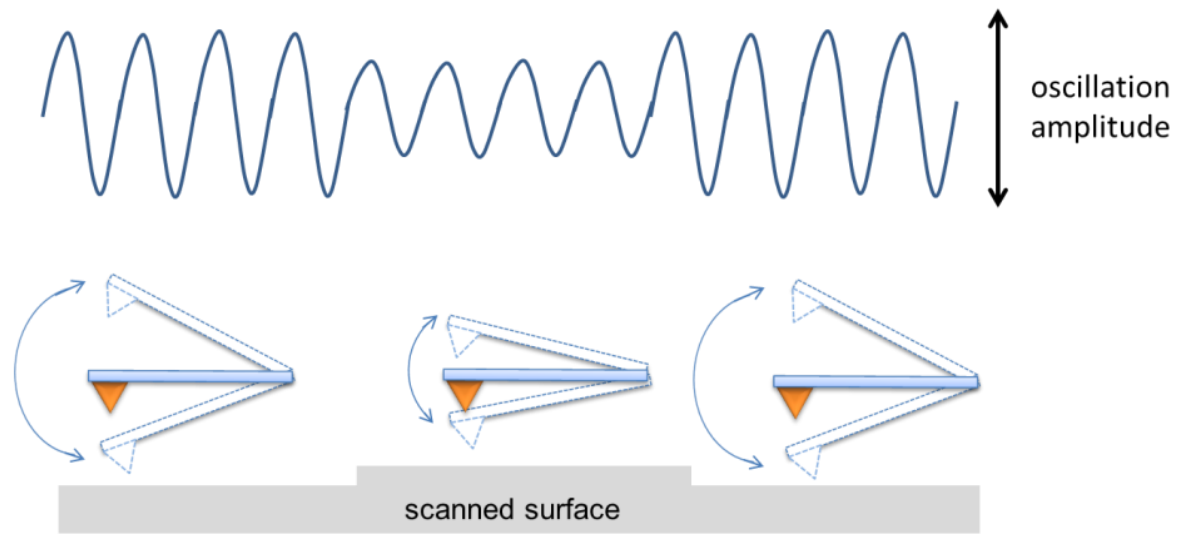

Figure 2.11 Schematic illustrations of (a) the basic principle of AFM and (b) the tapping- mode AFM

In the AFM experiments presented in Chapter 5, an Agilent 5500 device is operated under tapping mode. The environmental control chamber enables the protection of the samples in a controlled $\mathrm{N}_{2}$ atmosphere. The cantilever with a typical resonance frequency of $250-300 \mathrm{kHz}$ and 
a nominal spring constant around $40 \mathrm{~N} / \mathrm{m}$ is chosen for the imaging. Then, a tiny droplet (with a diameter of several millimeters) of the isotropic sample is placed onto a DMOAP-coated substrate. The temperature controlled by a Lakeshore device and is slowly decreased (at a rate of $0.05{ }^{\circ} \mathrm{C} / \mathrm{min}$ ) from the isotropic range to the smectic range of the liquid crystal. Once the temperature reaches the target, it remains constant during the AFM measurements. Both the surfaces of the macroscopic droplet and the precursor film spreading at the foot of the droplet are characterized by tapping-mode AFM. The height and phase images are recorded for each measurement.

\subsection{Theoretical Models}

\subsubsection{Landau-de Gennes Theory}

To theoretically interpret the nematic surface order, a Landau-de Gennes model of Sheng [46] is employed in this dissertation. This model is actually in the framework of the Landau-de Gennes theory which is a phenomenological model for the description of the nematic-isotropic transition. This theory is relatively simple but it can capture the most important elements of the nematic-isotropic transition. In the following, the general Landau-de Gennes theory is first introduced.

The phenomenological approach to the phase transitions neglect the details of molecular structure and molecular interactions. The key issue in such a theoretical framework is the change in symmetry and corresponding order parameters at a phase transition. By assuming that the order parameter $S$ is small in the nematic phase in the vicinity of the nematic-isotropic transition, the free energy density $g$ can be expended in a power series of $S$ :

$$
g=g_{\text {iso }}+\frac{a}{2}\left(T-T^{*}\right) S^{2}-\frac{1}{3} B S^{3}+\frac{1}{4} C S^{4},
$$

where $g_{i s o}$ is the free energy density of the isotropic phase $(S=0), a, B$, and $C$ are the coefficients of the usual Landau series expansion and set positive, and $T^{*}$ gives the stability limit of the isotropic phase [52]. The equilibrium value of $S$ should give the minimum value for the free energy, so the above expression is differentiated with respect to $S$ and the derivative is set equal to zero 


$$
\left(\frac{\partial g}{\partial S}\right)_{T}=a\left(T-T^{*}\right) S-B S^{2}+S^{3}=0 .
$$

The solutions are

$$
S=0 \text { or } S=\frac{B \pm \sqrt{B^{2}-4 a C\left(T-T^{*}\right)}}{2 C}
$$

The solution $S=0$ corresponds to a minimum of the free energy of the isotropic phase. Then, for the other two values, if one gives a minimum of the free energy, the other leads to a maximum of the free energy which should be suppressed. At the transition temperature $T_{\mathrm{c}}$, the free energies of the isotropic and the nematic phases are required to be equal, i.e., $g=g_{i s o}$. Then,

$$
S=0 \text { or } \frac{a}{2}\left(T-T^{*}\right)-\frac{1}{3} B S+\frac{1}{4} C S^{2}=0 .
$$

Combining the requirements that $g=g_{\text {iso }}$ and $(\partial g / \partial S)_{T_{c}}=0$ yields

$$
S_{c}=0, \text { or } S_{c}=\frac{2 B}{3 C}
$$

Then, the phase transition temperature $T_{c}$ can be solved as

$$
T_{c}=T^{*}+\frac{2 B^{2}}{9 a C}
$$

If the two solutions of the order parameter lead to minima of the free energy at $T_{c}$, the second derivative of the frees energy $g$ with respect to $S$ must be positive:

$$
\left(\frac{\partial^{2} g}{\partial S^{2}}\right)_{T_{C}}=a\left(T_{c}-T_{*}\right)-2 B S+3 S^{2}=\frac{2 B^{2}}{9 C} .
$$

Obviously $2 B^{2} / 9 C>0$, so both $S_{c}=0$ and $S_{c}=2 B / 3 C$ satisfy the positive requirement of the second derivative of $g$ at $T_{c}$. The nematic-isotropic phase transition involves a discontinuity in the order parameter which manifests the observed first-order phase transition.

\subsubsection{Modeling of the Nematic Surface Order}


In the Landau-de Gennes model developed by Sheng for nematic surface order [46], two more contributions are implemented into the expression of the free energy density as shown above. By taking the influence of the surface into account, the Sheng's model is expressed as

$$
g=g_{\text {iso }}+\frac{a}{2}\left(T-T^{*}\right) S^{2}-\frac{1}{3} B S^{3}+\frac{1}{4} C S^{4}-V \delta(z) S+\frac{L}{2}\left(\frac{\partial S}{\partial z}\right)^{2},
$$

where $V$ is the strength of the surface potential which is coupled to the nematic order parameter $S$ at the surface, $z$ is the distance from the surface. The last term in the expression with the coefficient $L$ designates the elastic energy corresponding to a $z$ variation of $S$. The next to last term leads to a non-zero value of $S$ at the surface $(z=0)$ even at temperatures above the bulk transition; the magnitude of this non-zero surface value is determined by $V$. By minimization of the free energy, the order parameter profile $S(z)$ is obtained as a function of temperature. The procedure of minimizing the free energy and obtaining $S(z)$ follows the description shown in $[46,88,90,115]$ and is briefly described here.

The free energy per unit area $F$ is obtained by integration of $g$ over $z$ :

$$
F=\int_{0}^{\infty} g\left(S, \frac{d S}{d z}\right) d z
$$

To determine the equilibrium form of $S(z)$, the condition of minimum free energy is employed. In the isotropic phase, i.e., for $T>T_{c}$, the value of the order parameter in the bulk is $S(z \rightarrow \infty)=$ 0 . However, because of the formation of an anisotropic nematic wetting layer at the interface, the order parameter near the interface is larger than zero, $S(z) \neq 0$. Exactly at the interface, i.e., for $z=0$, the order parameter is denoted as $S_{0}$. The value of $S(z)$ decreases with increasing the distance from the interface $z$ and finally disappears for large $z$. In the isotropic phase, $S(z)$ is given by:

$$
\sqrt{\frac{1}{2 L}} z=\operatorname{sign}\left(S-S_{0}\right) \int_{S_{0}}^{S} \frac{1}{\sqrt{\frac{a}{2}\left(T-T^{*}\right) S^{2}-\frac{1}{3} B S^{3}+\frac{1}{4} C S^{4}}} d S .
$$

An analytical solution for $S(z)$ has been given by Tarczon and Miyano [88]. The order parameter profile $S(z)$ can be determined by the following terms: 


$$
\begin{gathered}
S(z)=\frac{2 a\left(T-T^{*}\right) Z}{Z^{2}+\frac{3}{2} B Z+\frac{1}{9} B^{2}-\frac{a}{2} C\left(T-T^{*}\right)}, \\
Z=Z(z)=R\left(S_{0}\right) e^{\frac{z}{\xi}}, \\
\xi=\sqrt{\frac{L}{a\left(T-T^{*}\right)},} \\
R\left(S_{0}\right)=\frac{1}{S_{0}} \sqrt{2 a\left(T-T^{*}\right)\left[\frac{a}{2}\left(T-T^{*}\right)-\frac{1}{3} B S_{0}+\frac{1}{4} C S_{0}^{2}\right]}+\frac{a}{S_{0}}\left(T-T^{*}\right)-\frac{1}{3} B .
\end{gathered}
$$

A numerical treatment of the equation

$$
\frac{a}{2}\left(T-T^{*}\right) S_{0}{ }^{2}-\frac{1}{3} B S_{0}{ }^{3}+\frac{1}{4} C S_{0}{ }^{4}=\frac{V^{2}}{2 L}
$$

can lead to many values of $S_{0}$. The solution for $S_{0}$ is determined by the value at which $F_{\mathrm{g}}$ is the minimum,

$$
F_{g}=\operatorname{sign}\left(S_{0}\right) \sqrt{2 L} \int_{0}^{S_{0}} \sqrt{\frac{a}{2}\left(T-T^{*}\right) S_{0}{ }^{2}-\frac{1}{3} B S_{0}{ }^{3}+\frac{1}{4} C S_{0}{ }^{4}} d S-V S_{0}
$$

The experimental results are reproduced with a single set of parameters $a, B, C$, and $L$, with values known for $\mathrm{nCB}$ compounds [116-117] and only the value of $V$ is varied in order to describe the influence of the properties of the liquid crystal interfaces.

The order parameter profile $S(z)$ can be transformed into profiles for the ordinary refractive index $n_{o}(z)$ and the extraordinary refractive index $n_{e}(z)$ as described in [90], i.e.,

$$
\begin{aligned}
& n_{o}^{2}(z)=n_{f}^{2}+d_{\zeta}(z)\left\{\left[n_{\text {iso }}^{2}-\frac{1}{3} \Delta \epsilon_{\max } S(z)\right]-n_{f}^{2}\right\} \\
& n_{e}^{2}(z)=n_{f}^{2}+d_{\zeta}(z)\left\{\left[n_{i s o}^{2}+\frac{2}{3} \Delta \epsilon_{\max } S(z)\right]-n_{f}^{2}\right\}
\end{aligned}
$$


with $d_{\zeta}(z)=1 / 2[1+\tanh (z / \zeta)]$ introducing a surface roughness of width $\zeta, n_{\text {iso }}$ being the refractive index of the isotropic phase of the nematic liquid crystal, $\Delta \epsilon_{\max }=\left(n_{e}^{2}-n_{o}^{2}\right)_{\max }$ describing the birefringence of the nematic phase of perfect order $(S=1)$, and $n_{f}$ being the refractive index of the fluid phase in contact with the liquid crystal. For the fluid studied in Chapter 3 , the value of $n_{f}$ depends on the water-to-glycerol ratio; for the liquid crystal/air interfaces as presented in Chapter $4, n_{f}=1$.

The continuous profiles $n_{o}(z)$ and $n_{e}(z)$ are then approximated by a multilayer system with several hundred birefringent layers possessing constant refractive indices. The orientation of the optical axis of each layer is perpendicular to the layer plane. The value of $\bar{\rho}(T)$ is then calculated using standard Fresnel formulae and the recursive formalism of Crook [113]. The calculated $\bar{\rho}(T)$ data are fitted to the measured $\bar{\rho}(T)$ data by varying the value of $V$. 


\section{Chapter 3. Surface Order at Surfactant-Laden Interfaces between Isotropic Liquid Crystals and Liquid Phases with Different Polarity}

\subsection{Introduction}

This chapter concerns an ellipsometric study of the surface order at surfactant-laden interfaces between thermotropic liquid crystals and liquid phases the polarity of which can be varied. One motivation of this study is to explore whether the rich surface ordering behavior observed at liquid crystal/water interfaces [41-43] can be extended to interfaces between liquid crystals and other fluids, since in practice the immiscible fluid can be a non-water phase or a phase in which water is just one of the several components. Another motivation is to investigate how the surface ordering behavior of liquid crystals responds to a change of the bulk property of the immiscible phase in contact. Recently, Carlton et al. have reported the anchoring behavior of a nematic liquid crystal in contact with aqueous phases whose bulk properties such as $\mathrm{pH}$ values and ionic compositions can be changed [118-119]. It has been shown that changing the bulk properties of the aqueous phase results in different anchoring behavior of the nematic liquid crystal. Herein, binary mixtures of water and glycerol are employed as the immiscible phase to create interfaces with the liquid crystals doped with a surfactant (monoolein) of a fixed concentration. Glycerol has often been used as an immiscible fluid for the study of interfacial phenomena of liquid crystals [120]. Considering the fact that the relative dielectric constant of glycerol $\left(\varepsilon_{r}=41\right.$ at $\left.20^{\circ} \mathrm{C}\right)$ is considerably smaller than the value of water $\left(\varepsilon_{r}=80\right.$ at $\left.20{ }^{\circ} \mathrm{C}\right)$, the variation of the glycerol-to water ratio corresponds to a systematic variation of the polarity of the immiscible fluid phase. For instance, in a 1:1 mixture (equal volumes) of water and glycerol, $\varepsilon_{r}=63.6$ at 20 ${ }^{\circ} \mathrm{C}$ [121]. Thus, a variation of the concentration of glycerol in the mixture corresponds to the change of the polarity of the immiscible liquid phase. The smectic or nematic order at the interfaces is determined by an ellipsometer as the polarity of the immiscible phase is changed.

The two liquid crystal compounds under investigation were $9 \mathrm{CB}$ and $12 \mathrm{CB}$, which display isotropic-nematic and isotropic-smectic bulk transitions, respectively. Their phase sequences 
and transition temperatures are shown in Table 1.1 in Chapter 1. The liquid crystal compounds were doped with a small amount of monoolein with mole fractions of 0.005 (9CB sample) and 0.016 (12CB sample), respectively. The experimental setup for the ellipsometric study of the surface order at interfaces between liquid crystals and fluids and the general experimental procedures can be also found in Chapter 2. For the measurements of the temperature dependence of the ellipsometry coefficient $\bar{\rho}$, data are continuously collected while the temperature is changed at a slow constant rate (typically $20 \mathrm{mK} / \mathrm{min}$ ). The magnitude of $\bar{\rho}$ can be considered as a linear measure of the thickness of a nematic or smectic film as long as the film thickness is below $\approx 50 \mathrm{~nm}$ [89]. In order to investigate the influence of the polarity of the immiscible fluid phase, the surfactant-doped liquid crystal sample was kept constant whereas the immiscible fluid phase is changed with respect to the different volume concentration of glycerol: After each measurement, the liquid crystal sample was cooled into the solid state and the immiscible phase is replaced by a new water/glycerol mixture.

\subsection{Results and Discussion}

\subsubsection{Smectic Surface Order at Interfaces of Isotropic 12CB}

The liquid crystalline compound 12CB has only a single mesophase, i.e., the smectic- $A$ phase. The pretransitional ordering behaviors of isotropic 12CB at air [11], solid [12] or water [42] interfaces have been investigated. Usually when the surface field is sufficiently strong, one observes the growth of the smectic surface phase in a layer-by-layer manner. In a previous study, it was shown that apart from the layer-by-layer growth of single smectic layers, the surface order of the isotropic $12 \mathrm{CB}$ at the surfactant-laden interface can display multiple-layer thickness jumps at lower surfactant concentrations [42].

Figure 3.1a shows the temperature dependence of the ellipticity coefficient $\bar{\rho}$ of the interface of the monoolein-doped $12 \mathrm{CB}$ sample (mole fraction of monoolein $=0.016$ ) to pure water. The layer-by-layer growth of the single smectic layers indicates that such an interface can induce homeotropic anchoring. And the strength of the homeotropic anchoring is rather strong as evidenced by the fact that the temperature for the formation of the first single smectic layer is 

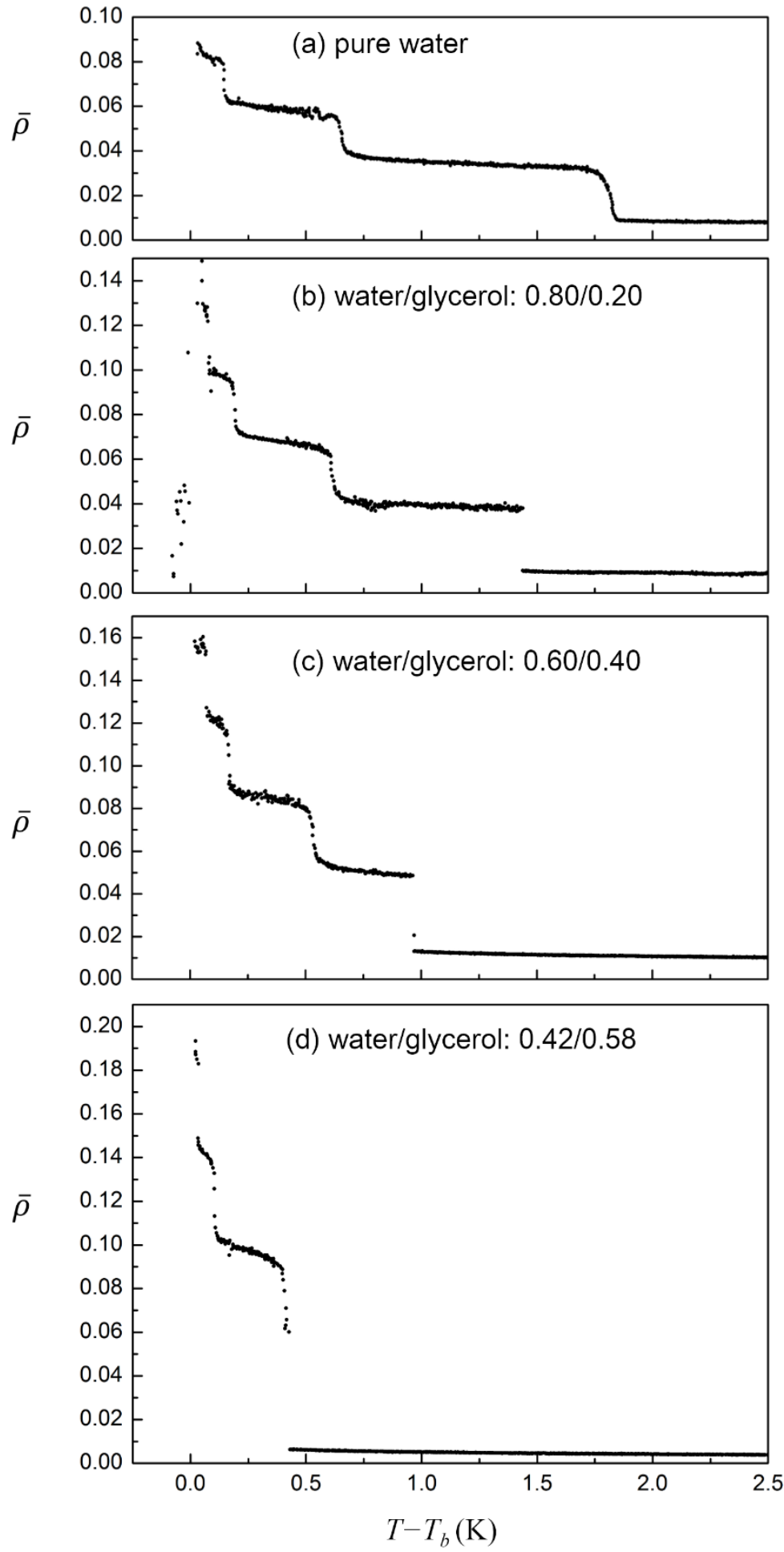

Figure 3.1 Temperature dependence of the ellipticity coefficient $\bar{\rho}$ of the interface of 12CB (doped with monoolein, mole fraction $=0.016$ ) to the water/glycerol mixtures with different glycerol content; the volume ratio between water and glycerol is shown in each panel. $T_{b}$ designates the bulk smectic-isotropic transition temperature of the sample. The stepwise increase of $\bar{\rho}$ with decreasing temperature indicates the formation of molecular smectic layers. 
$1.85 \mathrm{~K}$ above the bulk smectic-isotropic transition temperature $T_{b}$. Upon cooling from above, the second and third single smectic layers form at $0.7 \mathrm{~K}$ and $0.2 \mathrm{~K}$ above $T_{b}$, and the temperature for the formation of the forth layer and $T_{b}$ are difficult to be distinguished. When the pure water phase is replaced by a mixture of water and glycerol, the growth of the smectic surface phase behaves distinctly. The results shown in Figure 3.1b-d are from the ellipsometric measurements of the interfaces between the monoolein-doped 12CB sample and the water/glycerol mixtures of different glycerol concentrations. The first observed effect from the mixing of glycerol on the smectic surface order is the decrease of the temperature for the formation of the first single smectic layer. Second, as the temperature at which the first single smectic layer grows is decreased towards to the bulk transition, the first and the second single smectic layers transitions merge into a thickness jump consisting of two smectic layers. This behavior is evidenced by the result from the liquid phase containing 58\% glycerol (see Figure 3.1d). The two effects exerted by adding glycerol into water on the surface ordering behavior of $12 \mathrm{CB}$ resemble those found from decreasing the surfactant concentration in 12CB [42]. The third remarkable effect from increasing the glycerol content in the liquid phase turns out to be the magnitude of the $\Delta \bar{\rho}$ steps which corresponds to the formation of one molecular smectic layer: the value of $\Delta \bar{\rho}$ is increased by an increase of the glycerol concentration. To explain this observation, one must consider the optical properties of the liquid phase that depends on the compositions of water and glycerol. The magnitude of the $\Delta \bar{\rho}$ steps for a single smectic layer can be constant only if the refractive indices of the two immiscible phases do not change. However, the refractive index of the water/glycerol mixture has been found to be increased by an increase of the glycerol concentration [122]. Thus, the observation of the variation of the values of $\Delta \bar{\rho}$ for a single smectic layer in Figure 3.1 does not indicate the change of the smectic layer spacing but just indicates an effect from the change of the refractive index of the water/glycerol mixture.

The above-mentioned results show that the surface ordering behavior at the surfactant-laden $12 \mathrm{CB}$ interface is strongly influenced by the polarity of the immiscible liquid phase in contact. As the surfactant concentration, the polarity of the immiscible liquid phase is another tunable parameter to control the strength of the surface field. The influence of the surface field on the temperature of the layering transitions is more pronounced for the formation of the first smectic layer (this layer can be a single molecular smectic layer or consist of multiple molecular smectic layers) than for the formation of the subsequent smectic layers. The observations and the 

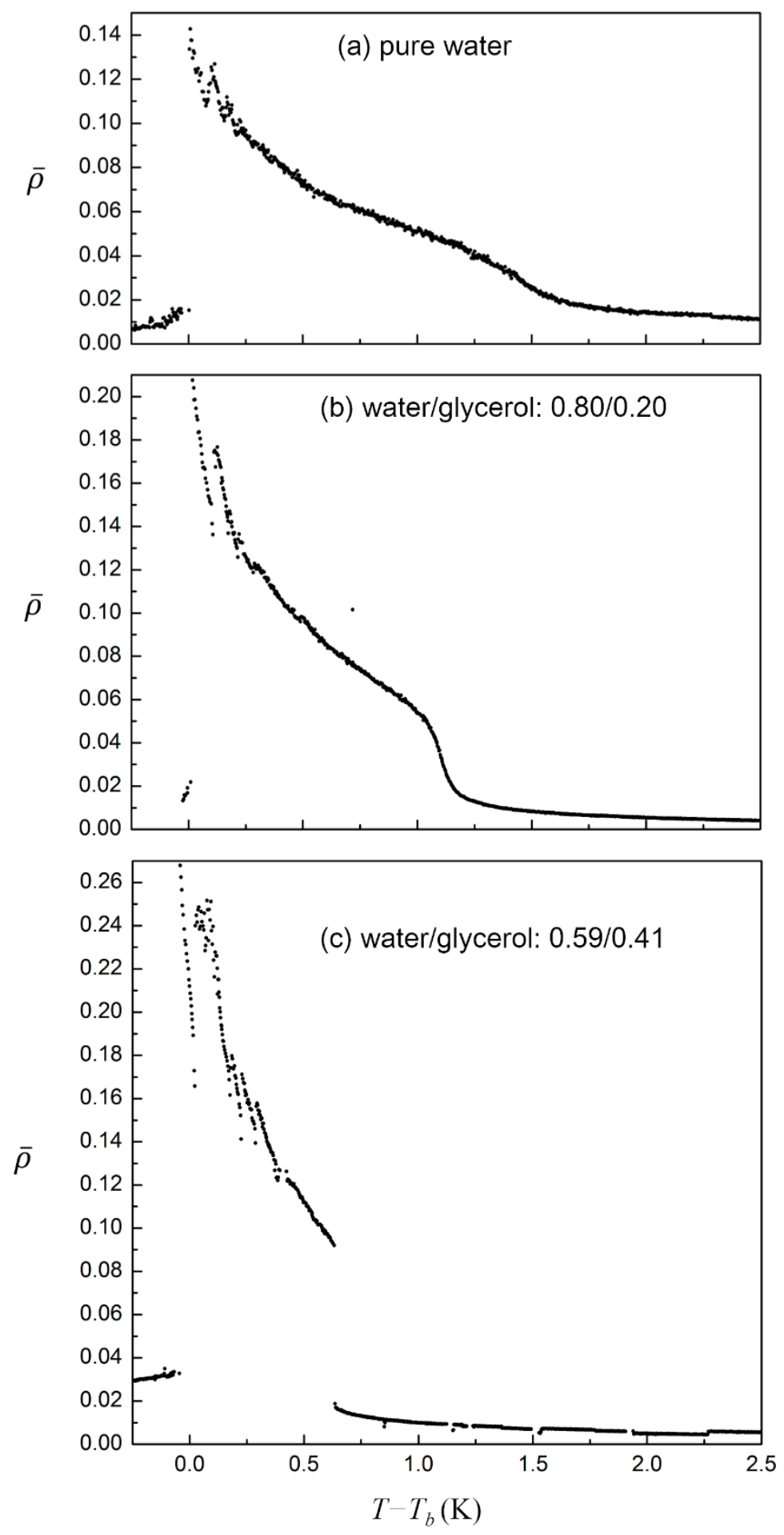

Figure 3.2 Temperature dependence of the ellipticity coefficient $\bar{\rho}$ of the interface of 9CB (doped with monoolein, mole fraction $=0.005$ ) to the water/glycerol mixtures with different glycerol content; the volume ratio between water and glycerol is shown in each panel. $T_{b}$ designates the bulk nematic-isotropic transition temperature of the sample. A prewetting transition occurs at the temperature $0.6 \mathrm{~K}$ above $T_{b}$ when the volume concentration of glycerol is 0.41 in the water/glycerol mixture, which is evidenced by the discontinuity in the $\bar{\rho}$ vs $T$ curve. 
the results from the previous study [42] indicate that the nature of the ordering effect from the surfactant molecules is short-range. A more quantitative discussion on the relationship between the polarity of the immiscible liquid phase and the surface ordering behavior is presented later in Subsection 3.2.3.

\subsubsection{Prewetting Behavior at Interfaces of Isotropic 9CB}

The liquid crystalline compound $9 \mathrm{CB}$ has an isotropic-nematic transition and hence at interfaces which induce homeotropic anchoring a thin nematic surface film should be present in the isotropic temperature range. If the strength of the homeotropic anchoring is sufficiently strong, the thickness of the nematic wetting layer grows continuously as the temperature approaches the bulk isotropic-nematic transition. Figure 3.2a displays an example of the continuous growth of the thickness of the nematic wetting layer, which is evidenced by the temperature-dependent growth of the ellipticity coefficient $\bar{\rho}$ of the interface of the monooleindoped 9CB sample (mole fraction of monoolein $=0.005$ ) to pure water. The behavior of the growth of the nematic wetting layer is also investigated with respect to the variation of the polarity of the immiscible liquid phase. Figure $3.2 \mathrm{~b}$ is obtained from the ellipsometric measurement in which the pure water is replaced by a water/glycerol mixture (the volume concentration of glycerol is 0.20 ). It is obvious that mixing water with glycerol changes the feature of the $\bar{\rho}$ vs $T$ curve and the nematic surface order at the higher temperature side is less pronounced. Further increasing the concentration of glycerol in the mixture results in a discontinuity of the $\bar{\rho}$ value, as shown in Figure 3.2c. The discontinuous increase of the $\bar{\rho}$ value upon cooling in the $\bar{\rho}$ vs $T$ curve occurs at the temperature $0.6 \mathrm{~K}$ above the bulk isotropic-nematic transition $T_{b}$ when the volume concentration of glycerol is 0.41 . This is a signature of a prewetting transition at which the thickness of the nematic wetting layer undergoes a thin-thick transition. The prewetting transitions at surfactant-laden interfaces between 9CB and water have been observed by changing the surfactant concentration in the bulk 9CB [43]. Here, it is demonstrated again by the observation of the prewetting transition that the increase of the concentration of glycerol in the water/glycerol mixture has the same effect as the decrease of the surfactant concentration.

\subsubsection{Quantitative Discussion of the Influence of the Glycerol Content}


The results shown above demonstrate that an increase of the glycerol concentration in the water/glycerol mixture has qualitatively the same effects as a decrease of the monoolein concentration in the bulk phase of the liquid crystals. The influence of the glycerol concentration on the smectic and the nematic surface order can be understood more quantitatively. For the smectic surface order, the difference between the temperature at which the first smectic layer is formed $\left(T_{1}\right)$ and the bulk transition $\left(T_{b}\right)$ can be plotted as a function of the volume fraction of glycerol $\left(x_{\mathrm{v},}\right.$ glycerol $)$ in the immiscible phase. Figure 3.3a shows the linear relationship of the values of $T_{1}-T_{b}$ and $x_{\mathrm{v}}$, glycerol, with a slope of $-2.05 \mathrm{~K}$. The result here can be quantitatively compared with the data obtained from the change of the surfactant concentration [42]. For instance, an increase of $x_{\mathrm{v}}$, glycerol by $10 \%$ from 0 to 0.10 results in a decrease of $T_{1}-T_{b}$ by 0.205 $\mathrm{K}$. The decrease of $T_{1}-T_{b}$ by $0.205 \mathrm{~K}$, if achieved by decreasing the mole fraction of monoolein,
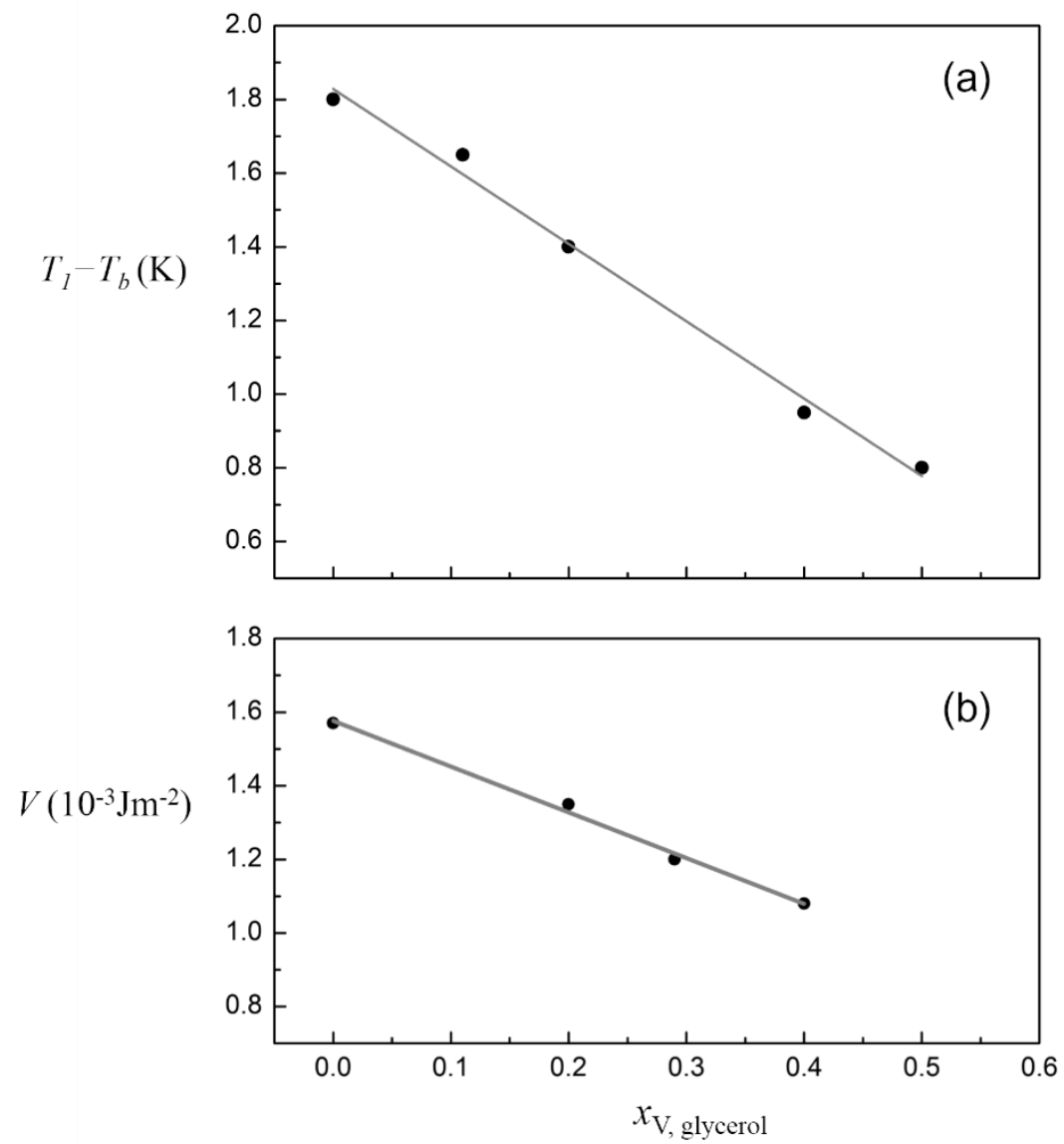

Figure 3.3 (a) Dependence of the temperature $T_{1}$, at which the first smectic layer is formed, on the volume fraction of glycerol in the water-glycerol phase $x_{\mathrm{v}, \text { glycerol. }}$ (b) Dependence of the effective surface field $V$ on the volume fraction of glycerol in the water-glycerol phase $x_{\mathrm{v}}$, glycerol. The value of $\mathrm{V}$ is determined by a fitting procedure (see Section 2.2 of Chapter 2) 
would require the change of the mole fraction of monoolein from 0.016 to 0.013 which corresponds to a decrease by $19 \%$. For the nematic surface order, the use of the Landau model of Sheng can provide a quantitative measure of the influence of the glycerol content. The Landau model assumes a linear coupling between the nematic order parameter and an effective surface field $V$. The value of $V$ can be determined by fitting calculated $\bar{\rho}(T)$ data to the measured $\bar{\rho}(T)$ curves. By this procedure (see Section 2.2 of Chapter 2), a value of $V$ is obtained from each $\bar{\rho}(T)$ vs $T$ curve. The dependence of $V$ on $x_{\mathrm{v}}$, glycerol in the water-glycerol mixture is shown in Figure $3 \mathrm{~b}$. From the $V$ vs $x_{\mathrm{v}}$, glycerol data, a decrease of $V$ by $1.29 \times 10^{-4} \mathrm{~J} / \mathrm{m}^{2}$ can be obtained by an increase of $x_{\mathrm{v}}$, glycerol from 0 to 0.1 . The same effect would be obtained if the monoolein mole fraction would be decreased from 0.005 to 0.0042 , corresponding to a decrease by $16 \%$, as estimated on the basis of the data in [43]. In short, a $10 \%$ increase of the glycerol content in the water/glycerol mixture corresponds to a decrease of the monoolein content in a range between $16 \%(9 \mathrm{CB})$ and $19 \%(12 \mathrm{CB})$.

Whereas a decrease of the surface coverage of the surfactant molecules due to a decrease of the surfactant bulk concentration is responsible for the weakening of the surface field in the previous reports [41-43], the question arises why an increase of the glycerol content in the water/glycerol mixture leads to the same effect. One might consider that glycerol has a stronger planar anchoring strength than water and hence suppresses the smectic or the nematic surface order (which requires the interface to induce homeotropic anchoring). However, experimental studies of thin nematic films on glycerol and water indicate that the planar anchoring strengths of both liquids are similar [120]. Therefore, it is unlikely that adding glycerol into water enhances the planar anchoring and suppresses the homeotropic anchoring which accounts for the weakening of the surface field. Another mechanism could be assumed is that a higher concentration of glycerol allows for a higher tendency of the diffusion of monoolein into the water/glycerol mixture; when the amount of monoolein is constant in the liquid crystal sample, this possible process might give rise to the decrease of the concentration in the liquid crystal sample after several measurements. In order to verify whether there is a remarkable diffusion process of monoolein from the liquid crystal phase into the water/glycerol phase, several experimental procedures were conducted. First, each ellipsometric measurement was repeated more than once and the result was found to be well reproducible. Second, after the measurement of the same $12 \mathrm{CB} /$ monoolein sample interfaced to a water/glycerol mixture with a relatively high 
glycerol content $\left(x_{\mathrm{v}}\right.$, glycerol $\left.=70 \%\right)$, the $12 \mathrm{CB} /$ monoolein sample kept immersed in this water/glycerol mixture for about two weeks, and then the immiscible phase was replaced by a pure water phase for a new measurement. The result of such a measurement is compared with the one obtained from the measurement of the same $12 \mathrm{CB} /$ monoolein sample interfaced to pure water prior to any contact with the water/glycerol mixtures. As displayed in Figure 4, the two results are well consistent with each other. Thus, the influence of the possible diffusion of the monoolein molecules is negligible. It is believed here that the change of the polarity of the water/glycerol phase affects the adsorption behavior of the monoolein molecules and hence the surface coverage at the interface. The detailed mechanism is discussed below.

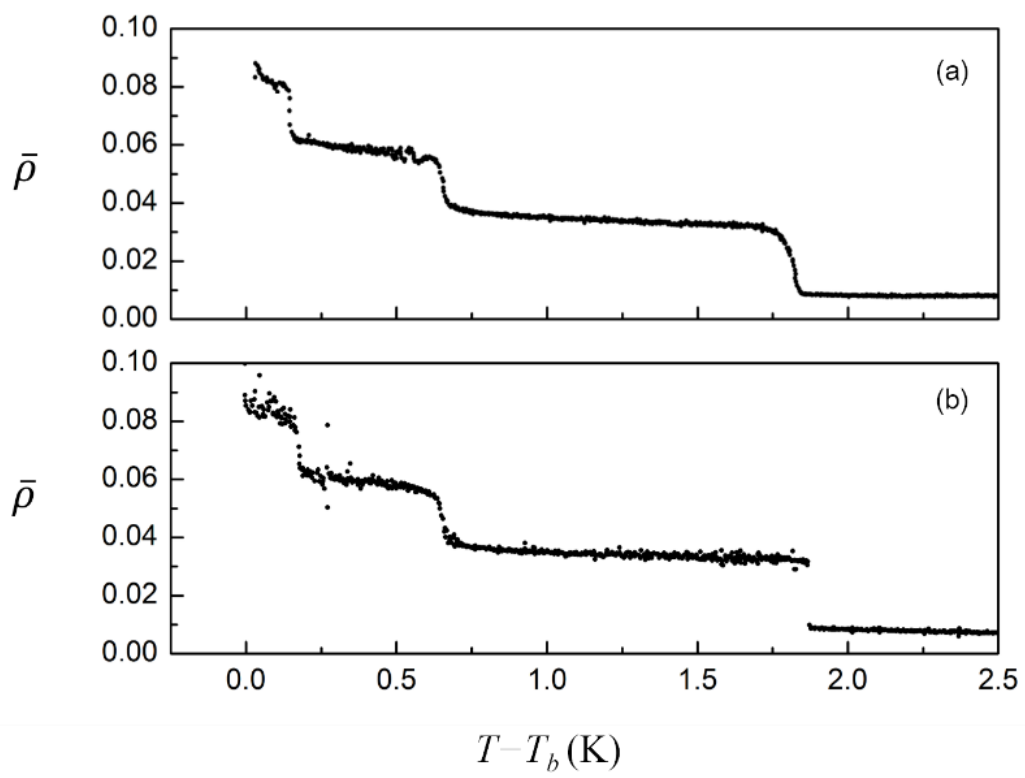

Figure 3.4 Temperature dependence of the ellipticity coefficient $\bar{\rho}$ of the interface of 12CB (doped with monoolein, mole fraction $=0.016$ ) to pure water. (a) Measurement done prior to any contact with the water/glycerol mixtures. (b) Measurement done after several measurements in which the water/glycerol mixtures were used and a long-time (about two weeks) immersion of the $12 \mathrm{CB} /$ monoolein in a water/glycerol mixture $\left(x_{\mathrm{v}}\right.$, glycerol $\left.=70 \%\right)$.

The adsorption of a surfactant is a process of transfer of surfactant molecules from the bulk solution phase to an interface or surface. There are many factors affecting the final surface coverage of surfactant molecules at a liquid/liquid interface, which include the nature of the surfactant molecules, the interactions of the constituent molecular blocks of the surfactant with 
the liquids, the bulk properties of the liquids, and so on. An important characteristic of a surfactant is the critical micelle concentration (CMC) that is defined as the concentration of the surfactant above which micelles are spontaneously formed. At the CMC, the surface coverage of the surfactant is at (near) a maximum value and the surfactant molecules begin to aggregate in the bulk phase. Below the CMC, the surface coverage of a surfactant is increased upon increasing the surfactant concentration in the bulk; above the $\mathrm{CMC}$, the surface coverage of the surfactant remains constant even if the surfactant concentration in the bulk is increased. The influence of the polarity of the liquid phase on the CMC of a given surfactant has been investigated for liquid/air interfaces in several studies where the tuning of the polarity has been achieved by using binary mixtures of water with ethylene glycol, glycerol, methanol or other alcohols, and other polar organic compounds of different ratios [123-129]. Those studies have found that the $\mathrm{CMC}$ increases with decreasing the polarity; that is, a decrease of the solvent polarity results in a decrease of the affinity of the surfactant to the interface and hence a decrease of the coverage of the interface. The experimental system presented here differs from those previous ones since the surfactant is dissolved in the liquid crystal phase but not in the aqueous phase whose polarity is varied. Still, the polarity difference between the two bulk liquid phases is an essential quantity that determines the affinity of a surfactant to an interface. Thus, decreasing the polarity of the immiscible liquid phase via increasing the glycerol content could be assumed to account for the weakening of the affinity of the monoolein molecules to the liquid crystal/liquid interface.

Adsorption isotherms which relate the surface coverage of surfactant molecules to the surfactant concentration in the bulk solution can be considered here to explain the equivalence of the variation of the polarity and of the surfactant concentration. A simple classical isotherm is the Langmuir isotherm [130]:

$$
\Gamma=\frac{K c}{1+K c}
$$

where $\Gamma$ denotes the dimensionless relative coverage (ranging from 0 to 1 ) and $c$ the surfactant bulk concentration. The constant $K$ describes the affinity of the surfactant to the interface; it can be considered as the ratio between the rates of surfactant adsorption and desorption. The Langmuir isotherm neglects the interactions between the adsorbed surfactant molecules. Another 
isotherm which takes into account possible interactions between the surfactant molecules is the Frumkin isotherm [131]:

$$
K c=\frac{\Gamma}{1-\Gamma} \exp (-2 \alpha \Gamma)
$$

where $\alpha$ is introduced for the description of attractive $(\alpha>0)$ or repulsive $(\alpha<0)$ forces between surfactant molecules. The Langmuir isotherms can be seen as a special case of the Frumkin isotherm when $\alpha=0$. In both isotherms, $\Gamma=0$ for $c=0$ and $\Gamma=1$ when $c$ is a larger value. When the polarity of the bulk water/glycerol phase is varied, the affinity constant $K$ is varied consequently. The effects exerted by the isotropic $9 \mathrm{CB}$ and $12 \mathrm{CB}$ on the value of $K$ can be assumed to be similar, considering the fact that they are homologues. It is not surprising that, when comparing the variation of the glycerol content and that of the surfactant concentration in both the systems of $9 \mathrm{CB}$ and $12 \mathrm{CB}$, one obtains similar relationships in both systems: a $10 \%$ increase of the glycerol content in the water/glycerol mixture corresponds to a decrease of the monoolein content of $16 \%$ in $9 \mathrm{CB}$ and $19 \%$ in $12 \mathrm{CB}$. This observation has demonstrated that the variation of $K$ has the equivalent effect on the surface coverage as the variation of $c$, which accounts for the tuning of the strength of the surface field.

\subsection{Conclusions}

The smectic and the nematic surface order at the surfactant-laden interfaces between isotropic liquid crystals and water/glycerol solutions have been studied. Whereas the concentration of the surfactant in the isotropic liquid crystal phase is kept constant, the volume fraction of glycerol in the water/glycerol phase is varied which corresponds to a systematic variation of the polarity of the immiscible phase in contact with the isotropic liquid crystal phase. Ellipsometry has been employed to probe the smectic layering transitions above the bulk isotropic-smectic transition of $12 \mathrm{CB}$ and the prewetting behavior above the bulk isotropic-smectic transition of $9 \mathrm{CB}$. The variation of the volume fraction of glycerol in the water/glycerol phase has the equivalent effect on the liquid crystal surface order as the variation of the surfactant concentration in the isotropic liquid crystal phase: an increase of the volume fraction of glycerol, similar to a decrease of the surfactant concentration in the isotropic liquid crystal phase, results in the suppression of the smectic or nematic surface order. By a quantitative comparison on the basis of the surfactant 
adsorption isotherms, it has been demonstrated that both of the two approaches leads to a systematic variation of the surface coverage of the surfactant molecules which accounts for the tuning of the strength of the surface field.

This study indicates that the concept of surface-induced pretransitional order of liquid crystals can be extended from the previously studied water interfaces to other fluid interfaces. Thus, the results are of practical interests for future studies of liquid crystal interfacial phenomena. The approach of changing the bulk properties of the immiscible liquid phase in contact with liquid crystals for tuning the surface field has the following advantages. First, it seems that only nonionic surfactants which are dissolved in the liquid crystal phase can induce the smectic surface order. A recent study has shown that the use of ionic surfactants in the water phase can leads to interfacial instability at the interface between a smectic liquid crystal and water [28]. In the previous study, the concentration of the surfactant is varied which means that the bulk liquid crystal phases of different surfactant concentrations are slightly different. Thus, the bulk property of the liquid crystal phase is not affected by a change of the immiscible liquid phase. Second, the experimental effort has been reduced since the surfactant-doped liquid crystal sample is kept constant and one just needs to change the immiscible liquid phase. 


\section{Chapter 4. Surface Ordering and Anchoring Behavior at Liquid Crystal Surfaces Laden with Semifluorinated Alkane Molecules}

\subsection{Introduction}

This chapter presents a study of the surface ordering and anchoring behavior at liquid crystal/air interfaces laden with semifluorinated alkane (SFA) molecules. As discussed in Chapter 1, although there have been a number of studies concerning the surface ordering and anchoring behavior at liquid crystal/air interfaces, no report has ever shown the manipulation of the behavior at such interfaces. In the experimental study presented in this chapter, the SFA, i.e., $\mathrm{C}_{18} \mathrm{H}_{37}-\mathrm{C}_{12} \mathrm{~F}_{25}$ (abbreviated as H18F12), is utilized as a kind of surface-active molecules which serves to tune the properties of liquid crystal/air interfaces. Due to a lower surface tension of the perfluorinated alkyl block of SFAs, this kind of molecules are able to reduce the surface tension of hydrocarbon solvents [47, 80-81]. Therefore, H18F12, when dissolved in a thermotropic liquid crystal, should be absorbed onto the air interface of the liquid crystal, in a similar way as usual surfactant molecules are absorbed at the surface of water.

Usually, molecular layers formed at liquid interfaces by adsorption of surface-active molecules from a bulk solution phase are commonly known as Gibbs films (see Chapter 1). The properties of Gibbs films of H18F12 molecules at air interfaces of several hydrocarbon solvents have been investigated by surface tension measurements and grazing incidence $x$-ray scattering [47]. It has been demonstrated that Gibbs films of $\mathrm{H} 18 \mathrm{~F} 12$ formed at free surfaces of hydrocarbon solutions exhibit sharp first-order phase transitions from a gas-like state at high temperatures to a condensed state at lower temperatures [47]. And the temperature at which the surface phase transition takes place depends on the bulk concentration of H18F12 in the solution. In the system of liquid crystals doped with H18F12 molecules, the concentrations of H18F12 in the liquid crystals can be controlled in a certain range so that the above-mentioned surface phase transitions of Gibbs films occur in the isotropic temperature range of the liquid crystals. Thus, such a system combines two different ordering processes at one interface: one is the growth of a nematic or smectic surface wetting layer and the other the structural phase transition in Gibbs 
films of H18F12. This system provides a platform for studying the interplay between two different surface ordering processes at the same interface. Another interesting aspect concerns the surface anchoring effect exerted by the Gibbs films on the liquid crystals. Depending on the nature of liquid crystal molecules, the intrinsic anchoring behavior at air interfaces can be varied (see Subsection 1.2.3 of Chapter 1). Since there are two different structural states for Gibbs films of H18F12 (i.e., gas-like state and condensed state), it is interesting to investigate how the anchoring behavior of liquid crystals responds to the structural phase transitions of Gibbs films.

The surface-induced pretransitional order at liquid crystal interfaces laden with SFA molecules was probed by Brewster's angle ellipsometry. The experimental configuration for the ellipsometric study of liquid crystal/air interfaces can be found in Chapter 2. The two liquid crystal compounds under investigation were $8 \mathrm{CB}$ and $12 \mathrm{CB}$, which display isotropic-nematic and isotropic-smectic bulk transitions, respectively. Their phase sequences and transition temperatures are shown in Table 1.1 in Chapter 1. The compound H18F12 was synthesized by the group of Prof. Martin Möller at RWTH, Aachen, Germany [132]. It was dissolved in the two liquid crystals with mole fractions $x_{\mathrm{HF}}$ between $10^{-5}$ and $10^{-3}$ by weighing appropriate amounts into small glass tubes and subsequent stirring for at least $1 \mathrm{~h}$ in the isotropic phase. For the measurements of the temperature dependence of ellipticity, the temperature was started from well above the bulk isotropic-liquid-crystalline transition (i.e., $T-T_{b}=15$ to $28 \mathrm{~K}$ ) and the values of $\bar{\rho}$ and $\theta_{B}$ were continuously collected while the temperature was changed at a slow constant rate ranging from 20 to $40 \mathrm{mK} / \mathrm{min}$. The magnitude of $\bar{\rho}$ can be considered as a linear measure of the thickness of a nematic or smectic film as long as the film thickness is below $\approx 50$ $\mathrm{nm}$ [89]. Regarding the detection of the anchoring behavior of liquid crystals, polarized light microscopy is utilized to observe the texture of thin liquid crystal films with one surface laden with H18F12 molecules (the air interface) and the other surface in contact with a DMOAPcoated glass substrate (the solid interface). The preparation of such liquid crystals films which are suitable for characterization of surface anchoring conditions is described in Subsection 2.1.2 of Chapter 2. Since the surface anchoring from the DMOAP-coated glass substrate is always homeotropic, a non-homeotropic anchoring at the second surface (the liquid crystal/air interface) can be detected by causing a non-zero transmission between crossed polarizers.

\subsection{Results and Discussion}




\subsubsection{Smectic Surface Order of 12CB Doped with H18F12}

First, the pretransitional ordering behavior at air interfaces of $12 \mathrm{CB}$ in the isotropic phase is considered by analyzing the ellipsometric data. The temperature dependence of the ellipticity coefficient at the free surface of a pure 12CB sample is designated by the black curve in Figure 4.1a. The stepwise increase of the $\bar{\rho}$ value is observed as the temperature is decreased towards the bulk isotropic-smectic transition $T_{b}$. The value of each step $\Delta \bar{\rho}$ can be transformed into the thickness of a single smectic layer, by the calculation on the basis of a simple slab model consisting of a birefringent 1 ayer (smectic $12 \mathrm{CB}$, ordinary refractive index $n_{o}=1.49$ and extraordinary refractive index $n_{e}=1.63$ ) [133] between two isotropic bulk media (air with $n=1$ and isotropic $12 \mathrm{CB}$ with $\left.n=\sqrt{\left(2 n_{o}^{2}+n_{e}^{2}\right) / 3}\right)$. And the calculated value of the thickness is 4.1 $\pm 0.1 \mathrm{~nm}$, which corresponds to the thickness of a single smectic layer of 12CB (previous X-ray studies have already shown a value of $3.91 \mathrm{~nm}$ [11]). Thus, the layer-by-layer growth of the smectic surface phase of $12 \mathrm{CB}$ is confirmed. And the temperature for the formation of the first smectic layer is rather high $\left(T-T_{b} \approx 24 \mathrm{~K}\right)$, implying a very strong homeotropic anchoring effect from the air interface.

When $12 \mathrm{CB}$ is doped with $\mathrm{H} 18 \mathrm{~F} 12$ with the mole fraction $x_{\mathrm{HF}}=0.6 \times 10^{-3}$, the layer-by-layer growth of smectic surface layers at the air interface is still observable but the temperature for the formation of the first smectic layer is already decreased (see the red curve in Figure 4.1a). This observation indicates that the adsorption of H18F12 molecules at the air interface reduces the strength of the homeotropic anchoring. As the bulk concentration of H18F12 in $12 \mathrm{CB}$ is increased, the effect of decreasing the temperature at which the first smectic layer is formed becomes more pronounced. Apart from this effect, when $x_{\mathrm{HF}} \geqslant 0.7 \times 10^{-3}$ a sharp drop of the $\bar{\rho}$ value occurs in each $\bar{\rho}$ vs $T$ curve close to the bulk transition, which indicates that the growth of the smectic wetting layer(s) is destroyed. The sudden decrease of the $\bar{\rho}$ value can take place 

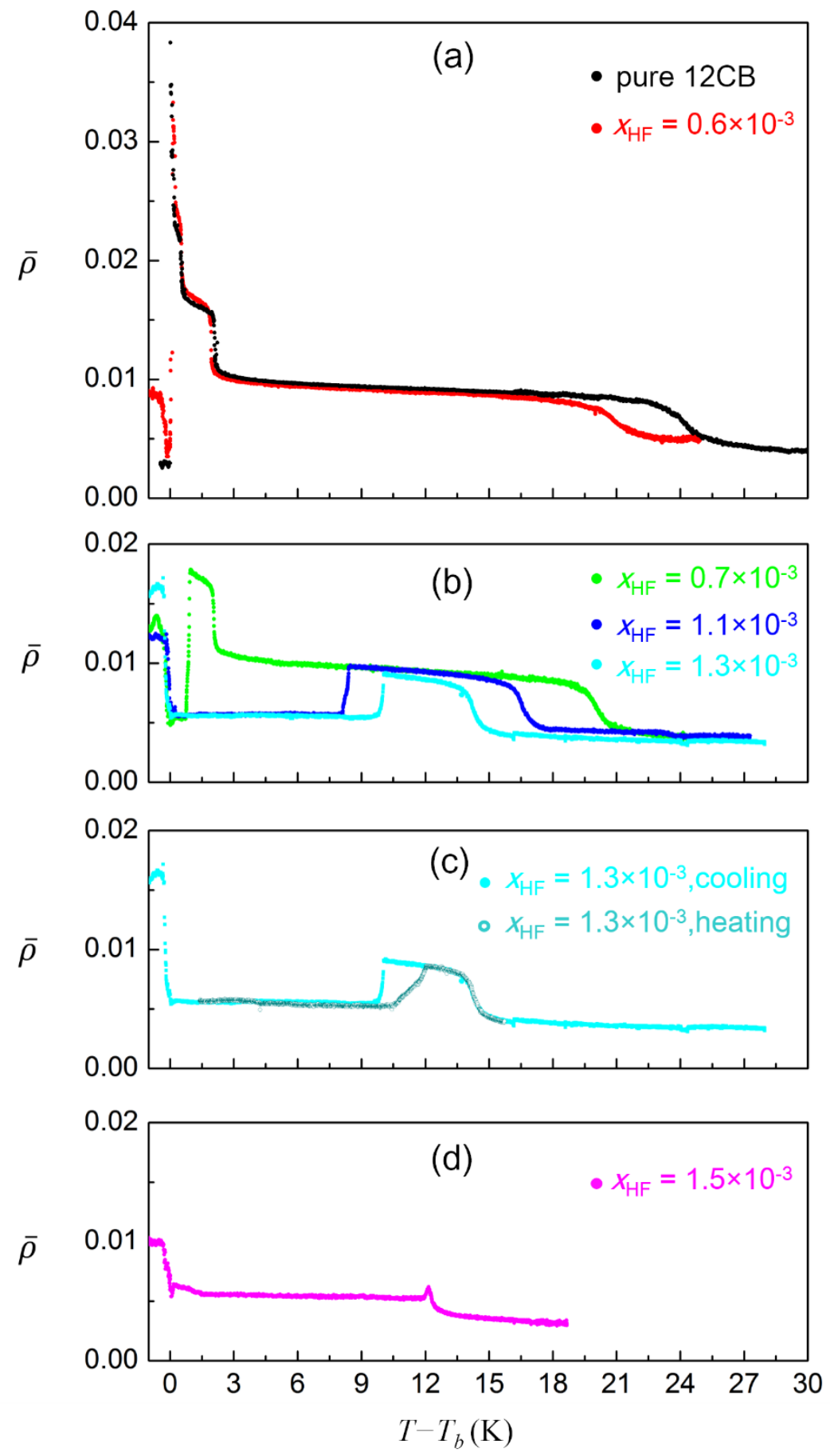

Figure 4.1 Temperature dependence of the ellipticity coefficient $\bar{\rho}$ of the air interface of 12CB doped with different amounts of $\mathrm{H} 18 \mathrm{~F} 12 ; T_{b}$ designates the bulk smectic-isotropic transition temperature of $12 \mathrm{CB}$. The different mole fractions of $\mathrm{H} 18 \mathrm{~F} 12, x_{\mathrm{HF}}$, are indicated by different colors in each figure. Part (c) shows the data from the cooling and heating runs for the H18F12-doped $12 \mathrm{CB}$ sample with $x_{\mathrm{HF}}=1.3 \times 10^{-3}$; note the observed thermal hysteresis. 
when the surface ordered phase consists of one or two smectic layer(s), as illustrated in Figure 4.1b. For example, for an H12F18-doped 12CB sample with $x_{\mathrm{HF}}=1.1 \times 10^{-3}$, one observes upon decreasing the temperature for first the formation of a single smectic layer at $T-T_{b} \approx 17 \mathrm{~K}$, followed by the destruction of this smectic layer at a $T-T_{b} \approx 8 \mathrm{~K}$. The temperature $T_{\mathrm{HF}}$, at which the sharp drop in the $\bar{\rho}$ vs $T$ curve occurs, increases with increasing the H18F12 concentration in the $12 \mathrm{CB}$ bulk solutions. This observation is in good agreement with the previous conclusion that the temperature for the structural phase transition of the Gibbs film of $\mathrm{H} 18 \mathrm{~F} 12$ depends on the concentration of H18F12 in the bulk solutions [47]. The first-order nature of the structural phase transition of the Gibbs film can be again demonstrated by the observation of the thermal hysteresis between the cooling and heating runs of a sample with $x_{\mathrm{HF}}$ $=1.3 \times 10^{-3}$ (see Figure 4.1c). An increase of $x_{\mathrm{HF}}$, on the one hand decreases the temperature for the growth of the first smectic layer, and on the other hand increases the temperature at which the destruction of the smectic layer(s) occurs. Thus, in the $\bar{\rho}$ vs $T$ diagram, the step-wise increase and the sudden drop of the $\bar{\rho}$ value move in the opposite directions along the temperature axis as $x_{\mathrm{HF}}$ is increased, and finally "meet" at a certain temperature. For $x_{\mathrm{HF}}=1.5 \times 10^{-3}$, the data indicate that the initial formation of smectic surface order is preempted by the transition in the H18F12 film at a temperature when $T-T_{b} \approx 12 \mathrm{~K}$ (see Figure 4.1d).

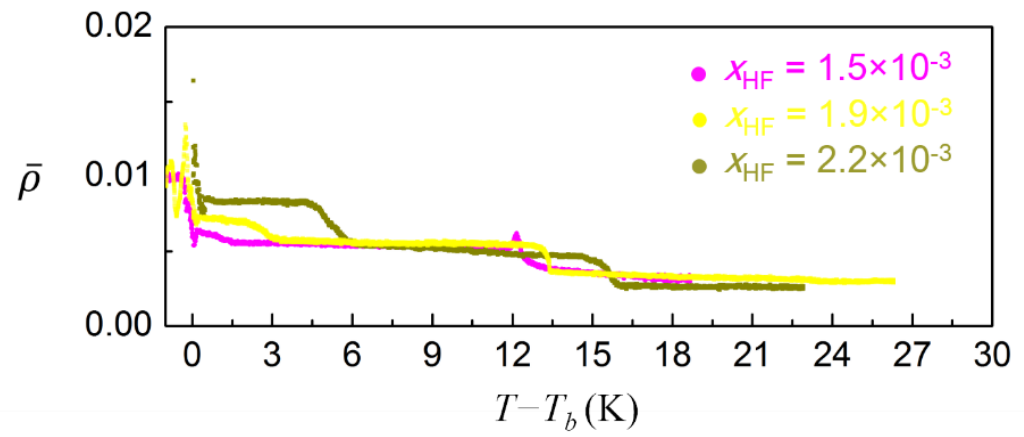

Figure 4.2 Temperature dependence of the ellipticity coefficient $\bar{\rho}$ of the air interface of H18F12-doped 12CB samples with $x_{\mathrm{HF}} \geq 1.5 \times 10^{-3}$. Note the additional increase of $\bar{\rho}$ near the bulk transition for the two samples with $x_{\mathrm{HF}}=1.9 \times 10^{-3}$ and $x_{\mathrm{HF}}=2.2 \times 10^{-3}$. 
If $x_{\mathrm{HF}}$ is further increased, the pretransitional smectic surface order of $12 \mathrm{CB}$ completely vanishes in the whole isotropic temperature range, because the possible temperature at which the first smectic layer is formed would be lower than the temperature at which the structural phase transition of the Gibbs film occurs. Thus, the change of the $\bar{\rho}$ value can result only from the structural change of Gibbs films of H18F12. For $x_{\mathrm{HF}}>1.5 \times 10^{-3}$, a tiny step-like increase of the ellipticity corresponding to $\Delta \bar{\rho}=0.002$ at the higher temperature side indicates that the structural phase transition from the Gibbs film is still present. Moreover, an additional increase of $\bar{\rho}$ in the temperature region near $T_{b}$ is observed for the samples with very high concentrations of H18F12 (see Figure 4.2). Such an additional increase of $\bar{\rho}$ was found to be arbitrary since the magnitude is not reproducible for several measurements on a given sample under the same experiential condition. This behavior may be due to the fact that the H18F12-doped 12CB samples with high concentrations of H18F12 are not homogeneous anymore when the temperatures are near $T_{b}$, thus resulting in a significant change of the optical properties of the bulk solutions.

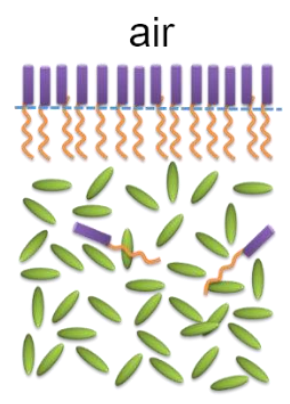

(c)

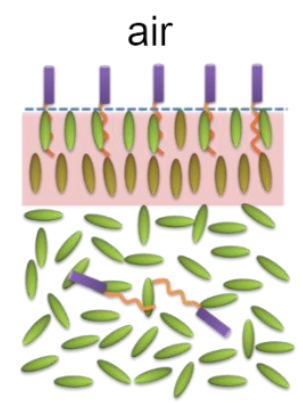

(b)

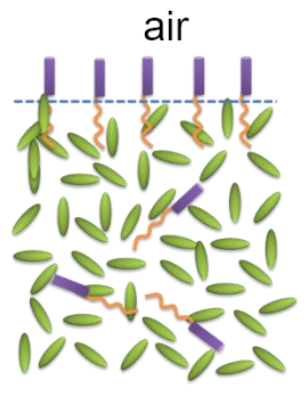

(a)

decreasing temperature

Figure 4.3 Schematic illustrations for the influence of the Gibbs film of H18F12 molecules on the smectic surface order at the air interface of $12 \mathrm{CB}$.

The results presented above show that both the dilute and the condensed states of the Gibbs film exert strong influence on the smectic surface order of $12 \mathrm{CB}$, which can be schematically illustrated by Figure 4.3. At a very high temperature (e.g., when $T-T_{b} \approx 28 \mathrm{~K}$ ), the air interface 
of $12 \mathrm{CB}$ can be regarded as isotropic, the situation of which corresponds to Figure 4.3a. The presence of a dilute Gibbs film weakens the strength of the homeotropic anchoring, but still allows for the formation of smectic surface layer(s), as illustrated by Figure 4.3b. However, as the temperature is decreased, the structural phase transition of the Gibbs film from the dilute state to the condensed state occurs and hence completely destroys the smectic surface layer. Thereby, at the air interface the smectic wetting layer is finally replaced by a condensed Gibbs film of SFA molecules, corresponding to Figure 4.3c. As discussed in Chapter 1, the core parameter for the study of surface-induced pretransitional order of liquid crystals is the strength of the surface field. It is obvious that Gibbs films of SFA are able to modify the surface field at air interfaces. Moreover, the condensed Gibbs films may induce planar anchoring to the liquid crystal, because for homeotropic anchoring the smectic surface order should be present. This assumption is further demonstrated by the following experimental investigations on the surface ordering of a nematic liquid crystal and by optical studies of the anchoring behavior of both smectic and nematic liquid crystal thin films.

\subsubsection{Nematic Surface Order of 8CB Doped with H18F12}

The black curve shown in Figure 4.4a is obtained from a measurement of the temperature dependence of the ellipticity at the free surface of pure $8 \mathrm{CB}$. The pronounced increase of $\bar{\rho}$ observed near the bulk transition $T_{b}$ indicates a divergence-like growth in thickness of a thin nematic wetting film as the temperature is decreased towards $T_{b}$. This result confirms the observations from earlier studies [91]. When $8 \mathrm{CB}$ is doped with $\mathrm{H} 18 \mathrm{~F} 12$, upon cooling, the value of $\bar{\rho}$ first increases continuously, then stops at a certain temperature and finally drops to a value of about 0.005 . The magnitude of $x_{\mathrm{HF}}$ in the $8 \mathrm{CB}$ samples is much smaller than that in the $12 \mathrm{CB}$ samples, because the temperature region under investigation in the case of $8 \mathrm{CB}$ is much lower than that in the case of $12 \mathrm{CB}$. The temperature $T_{\mathrm{HF}}$, at which the kink in the $\bar{\rho}$ vs $T$ curve occurs, also increases with increasing H18F12 concentration in the $8 \mathrm{CB}$ bulk phase, which is consistent with the observation from the $12 \mathrm{CB}$ samples. Thus, it is safe to conclude that, in the temperature region below the temperatures at which the kinks occur in the $\bar{\rho}$ vs. $T$ curves, the Gibbs films of H18F12 are in the condensed state. 
The influence of the formation of condensed Gibbs films on the nematic surface order is also remarkable, as evidenced by the sudden decrease of $\bar{\rho}$ at $T_{\mathrm{HF}}$. Several possibilities may cause the decrease: (i) Similar to the smectic surface layer(s), the homeotropically aligned nematic wetting
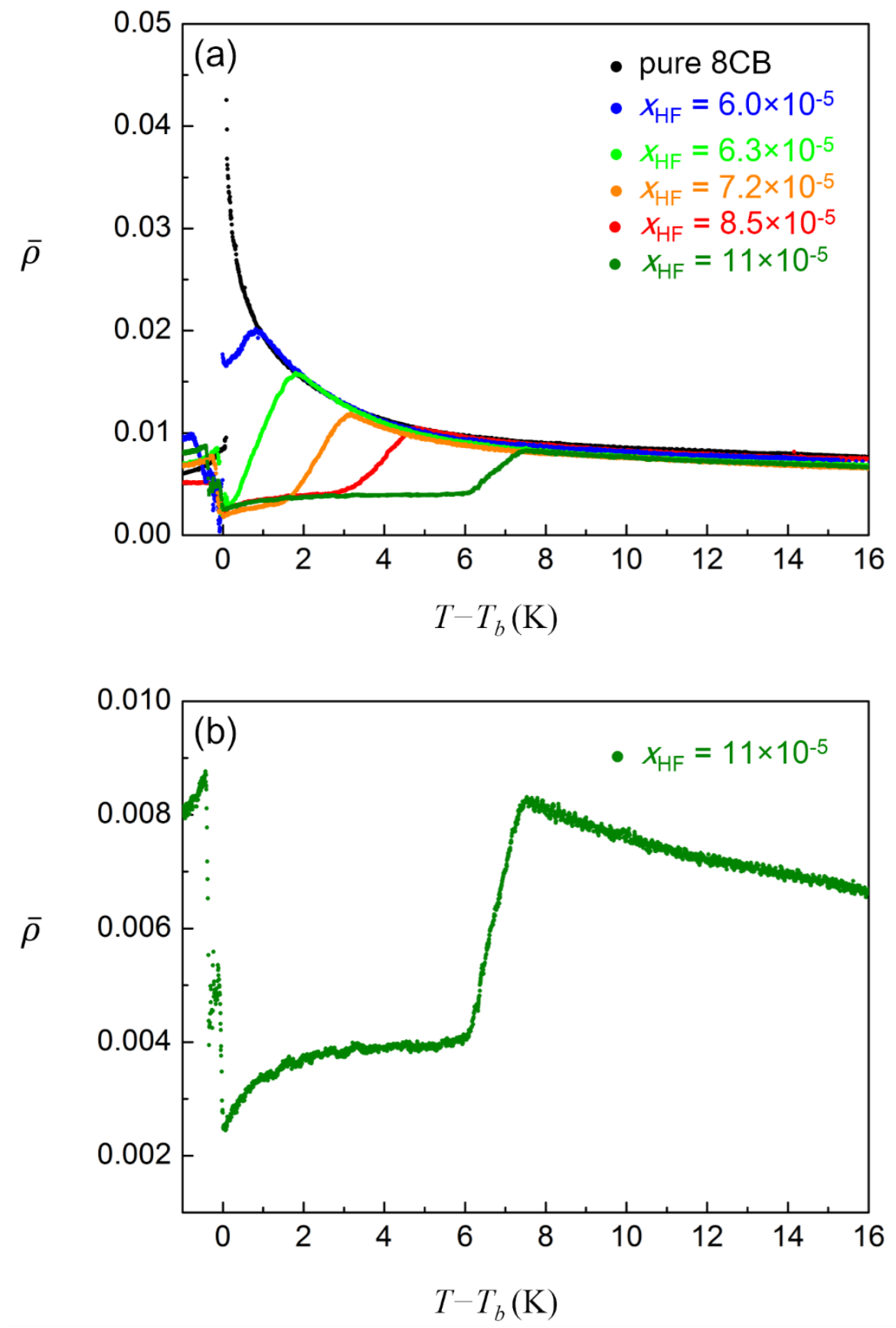

Figure 4.4 Temperature dependence of the ellipticity coefficient $\bar{\rho}$ of the free surface of $8 \mathrm{CB}$ doped with different amounts of $\mathrm{H} 18 \mathrm{~F} 12 ; T_{b}$ designates the bulk nematic-isotropic transition temperature of $8 \mathrm{CB}$. The different mole fractions of $\mathrm{H} 18 \mathrm{~F} 12, x_{\mathrm{HF}}$, are indicated by different colors. Part $\mathrm{b}$ shows the enlarged diagram of the data for $x_{\mathrm{HF}}=1.1 \times 10^{-4}$ with an expanded $\bar{\rho}$-scale; note the decrease of $\bar{\rho}$ as $T_{\mathrm{NI}}$ is approached from above. 
layer is completely destroyed by the formation of the condensed Gibbs film; (ii) The nematic wetting layer just reduces its thickness but is still homeotropically anchored; (iii) The liquid crystal molecules in the nematic wetting layer change their orientation from homeotropic to planar. By a careful examination of the ellipsometric data (including both the temperature dependences of $\bar{\rho}$ and $\theta_{B}$ ), the third assumption should account for the observation. First, when $T_{b}$ is approached, a second decrease of $\bar{\rho}$ in the vicinity of $T_{b}$ is observed, as clearly displayed in Figure $4.4 \mathrm{~b}$ with an expanded $\bar{\rho}$-scale. The second decrease of $\bar{\rho}$ indicates the possible presence of a planar nematic film below $T_{\mathrm{HF}}$ which grows in thickness as $T_{b}$ is approached. Second, the behavior of the Brewster's angle $\theta_{\mathrm{B}}$ clearly indicates that the anchoring of the nematic film changes at $T_{\mathrm{HF}}$ from homeotropic to planar.

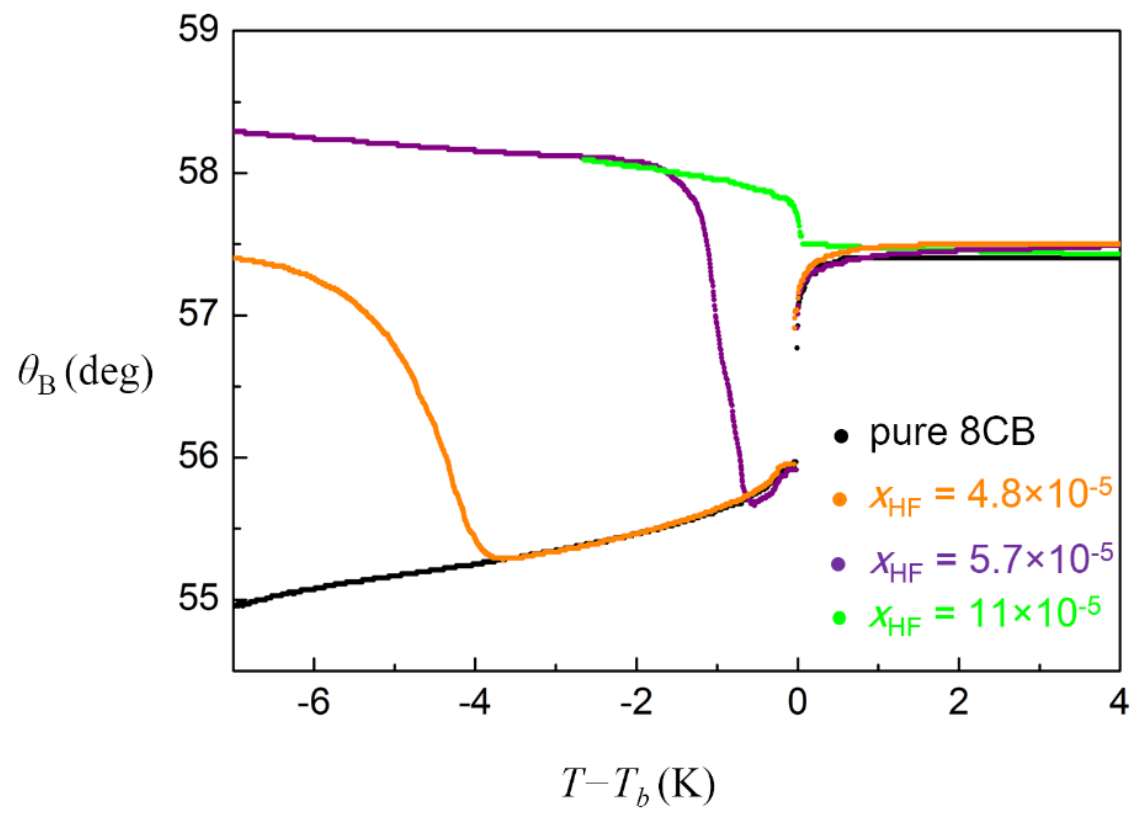

Figure 4.5 Temperature dependence of the Brewster's angle $\theta_{\mathrm{B}}$ of the free surface of $8 \mathrm{CB}$ doped with different amounts of $\mathrm{H} 18 \mathrm{~F} 12 ; T_{\mathrm{b}}$ designates the bulk nematic-isotropic transition temperature of $8 \mathrm{CB}$. The mole fractions of $\mathrm{H} 18 \mathrm{~F} 12$ are indicated by different colors.

The value of the Brewster's angle $\theta_{\mathrm{B}}$ is determined by the optical properties of the two bulk media (air and liquid crystal in this case). Calculating $\theta_{\mathrm{B}}$ for the surface of an isotropic liquid crystal with a refractive index $n_{i \text { so }}$ and a nematic liquid crystal with an ordinary index $n_{\mathrm{o}}$ and an 
extraordinary index $n_{\mathrm{e}}$ (being related as $n_{\text {iso }}=\sqrt{\left(2 n_{o}^{2}+n_{e}^{2}\right) / 3}$ and $\Delta n=n_{\mathrm{e}}-n_{\mathrm{o}} \approx 0.1$ ) yields a value for $\theta_{\mathrm{B}}$ that should be $\approx 2^{\circ}$ smaller for the nematic surface than for the isotropic surface provided that the optical axis of the nematic phase is oriented perpendicular to the surface. If the optical axis of the nematic liquid crystal is oriented parallel to the interface, $\theta_{\mathrm{B}}$ should be of similar magnitude or slightly larger (depending on the azimuthal in-plane orientation of the optical axis) than the value of the isotropic surface [134].

Figure 4.5 shows $\theta_{\mathrm{B}}$ as a function of $T$ for pure $8 \mathrm{CB}$ as well as several $\mathrm{H} 18 \mathrm{~F} 12$-doped $8 \mathrm{CB}$ samples. For pure $8 \mathrm{CB}, \theta_{\mathrm{B}}$ shows the expected drop by $\approx 2^{\circ}$ at $T_{\mathrm{b}}$, indicating the perpendicular orientation of the optical axis of the nematic phase. Below $T_{\mathrm{b}}, \theta_{\mathrm{B}}$ decreases continuously with decreasing $T$, reflecting the increase of $\Delta n$ in the nematic phase. Mixtures of $8 \mathrm{CB}$ and H18F12 with $x_{\mathrm{HF}}>6 \times 10^{-5}$ show a quite different behavior: at the transition temperature $T_{\mathrm{HF}}$ of the H18F12 film, $\theta_{\mathrm{B}}$ shows a slight increase and it increases again at $T_{\mathrm{b}}$ indicating that the optical axis is parallel to the surface. From these observations we can conclude that the dilute-tocondensed transition in the H18F12 film changes the orientation of the liquid crystal molecules at the interface from homeotropic to planar. This behavior is most clearly demonstrated if we tune the $\mathrm{H} 18 \mathrm{~F} 12$ concentration in the liquid crystal bulk phase to very low values so that $T_{\mathrm{HF}}$ is below $T_{\mathrm{b}}$ : for such samples, we first observe a decrease of $\theta_{\mathrm{B}}$ at $T_{\mathrm{b}}$ (the data coinciding with those of pure $8 \mathrm{CB})$ but $1 \mathrm{~K}\left(x_{\mathrm{HF}}=5.7 \times 10^{-5}\right)$ or $4 \mathrm{~K}\left(x_{\mathrm{HF}}=4.8 \times 10^{-5}\right)$ below $T_{\mathrm{b}}$ a pronounced increase of $\theta_{\mathrm{B}}$ occurs, indicating that the anchoring of the bulk nematic phase changes from homeotropic to planar.

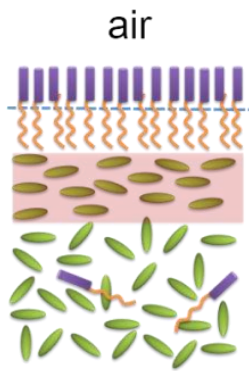

(b)

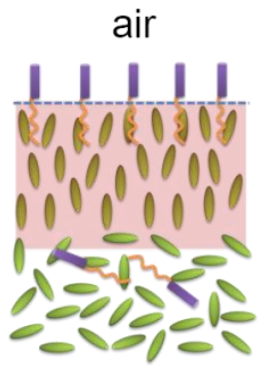

(a)

decreasing temperature

Figure 4.6 Schematic illustrations for the influence of the Gibbs film of H18F12 molecules on the nematic surface order at the air interface of $8 \mathrm{CB}$. 
The ordering process at the surface of isotropic $8 \mathrm{CB}$ laden with $\mathrm{H} 18 \mathrm{~F} 12$ molecules upon decreasing the temperature can be schematically illustrated in Figure 4.6. At a high temperature, the Gibbs film of H18F12 is in the dilute state which still allows the air interface to induce homeotropic anchoring to the liquid crystal. Thus, the air interface is found to be wet by a thin nematic surface layer of homeotropic anchoring, corresponding to Figure 4.6a. However, when the Gibbs film transforms into the condensed state at a lower temperature, the molecular orientation of the nematic wetting layer is changed to be planar, as shown by Figure 4.6b. The experimental observation of a pretransitional nematic surface order in the isotropic temperature range at interfaces inducing planar anchoring is rather rare, although it is theoretically expected for suitable surface potentials $[82,84]$. There are a few cases where the growth of a surface phase with negative birefringence has been observed on solid substrates with planar anchoring conditions [87-88], and the results of the present study may represent one more of these rare cases.

The nematic order at the surface of $8 \mathrm{CB}$ can be described by a Landau model of Sheng which assumes a linear coupling between the nematic order parameter and an effective surface field $V$. The value of $V$ can be determined by fitting the calculated $\bar{\rho}(T)$ data to the measured $\bar{\rho}(T)$ data. The detailed description of the Landau model and the fitting procedures is given in Chapter 2. The measured ellipsometric data for two samples, i.e., the pure 8CB sample and the one with $x_{\mathrm{HF}}=1.1 \times 10^{-4}$, are considered here. For the pure $8 \mathrm{CB}$ sample, when the value of $V$ is set to $3.0 \times 10^{-3} \mathrm{~J} / \mathrm{m}^{2}$, the calculated $\bar{\rho}(T)$ data can fit the measured $\bar{\rho}(T)$ data very well, as shown in Figure 4.7. For the H18F12-doped $8 \mathrm{CB}$ sample with $x_{\mathrm{HF}}=1.1 \times 10^{-4}$, the structural phase transition of the Gibbs film occurs at a temperature $6.3 \mathrm{~K}$ above the bulk nematic-isotropic transition (see Figure 4.4b). Thus, when $\mathrm{T}>T_{\mathrm{b}}+6.3 \mathrm{~K}$, the surface anchoring is still homeotropic and $V$ can be also set to $3.0 \times 10^{-3} \mathrm{~J} / \mathrm{m}^{2}$. However, for $\mathrm{T}<T_{\mathrm{b}}+6.3 \mathrm{~K}$, the surface induces planar anchoring to the liquid crystal wetting layer, in which the molecular axis are perpendicular to the surface normal. Since all in-plane orientations of the local director occur with equal probability, the macroscopic molecular axis is still parallel to the surface normal. Then, the nematic wetting layer has to be described as a layer of negative birefringence and hence the value of $\bar{\rho}$ decreases when the thickness of such a negatively birefringent film grows 
(see Figure 4.4b). The value of the surface field $V$ for the planar anchoring exerted by the condensed Gibbs film is obtained, from the comparison of calculated and measured $\bar{\rho}$ (see also Figure 4.7), as $0.6 \times 10^{-3} \mathrm{~J} / \mathrm{m}^{2}$. This value is much smaller than that for the homeotropic anchoring in the case of the pure $8 \mathrm{CB}$ sample. It should be noted that in the above mentioned modeling the contribution from the condensed Gibbs film to the value of $\bar{\rho}$ is neglected, considering the fact that the formation of the condensed Gibbs film changes the value of $\bar{\rho}$ by 0.002 (see Subsection 4.2.1) which is much smaller than the birefringent film nematic film.

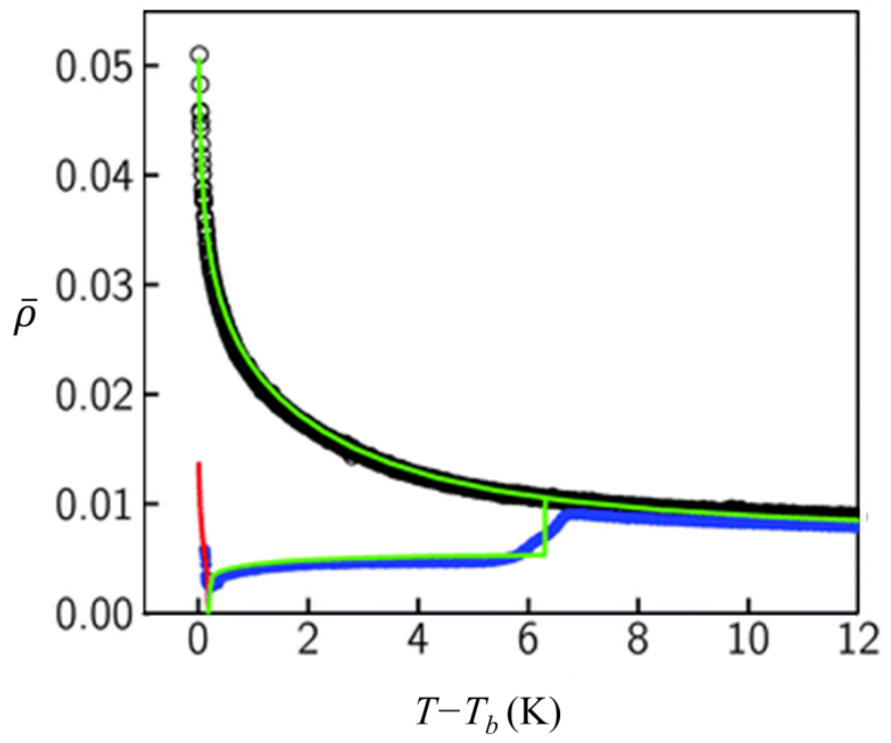

Figure 4.7 Temperature dependence of the ellipticity coefficient $\bar{\rho}$ measured for pure 8CB (०) and for an $8 \mathrm{CB}-\mathrm{H}_{18} \mathrm{~F}_{12}$ mixture with $x_{\mathrm{HF}}=11 \times 10^{-5}(\bullet)$. The green and red solid lines correspond to calculated values resulting from the Landau model (see text); the red values are in fact $<0$ and have been multiplied by -1 since our experimental setup does not determine the sign of $\bar{\rho}$.

\subsubsection{Surface Anchoring of Liquid Crystal Films Laden with H18F12 Molecules}

The analysis of the ellipsometric data obtained from the measurements on the air interfaces of $12 \mathrm{CB}$ and $8 \mathrm{CB}$ laden with $\mathrm{H} 18 \mathrm{~F} 12$ molecules indicates that the condensed Gibbs films of H18F12 molecules induce planar anchoring at the air interfaces. To demonstrate this effect more directly, polarized light microscopy is employed here to observe the textures of smectic or nematic films laden with H18F12 molecules. The observed textures can give evidence of the anchoring conditions from the two surfaces of the films. Detailed discussion on the principle of 
characterization of the surface anchoring of a liquid crystal film by polarized light microscopy is already presented in Chapter 2. In general, one surface of the liquid crystal film is in contact with a DMOAP-coated glass slide which induces strong homeotropic anchoring and the other surface of the film (which may be laden with the SFA molecules) is exposed to the air.

Figure 4.8a shows an optical microscopic image of a pure 12CB film pinned by the copper frames of a TEM grid. And the temperature is $1 \mathrm{~K}$ below the bulk smectic-isotropic transition. Since the two surfaces of the 12CB film induce homeotropic anchoring, the film almost appears to be dark under crossed polarizers. The occurrence of some small bright regions close to the edge of the frames is due to the perturbation from the copper frames. For an H18F12-doped $12 \mathrm{CB}$ sample with $x_{\mathrm{HF}}=1.1 \times 10^{-3}$, the ellipsometric measurement already confirms that the condensed Gibbs film of $\mathrm{H} 18 \mathrm{~F} 12$ is formed when $T-T_{b} \approx 8 \mathrm{~K}$ (see Figure $4.1 \mathrm{~b}$ ). Thus, at the temperature $1 \mathrm{~K}$ below $T_{b}$, the condensed Gibbs film is present and hence induces planar anchoring to the $12 \mathrm{CB}$ film at the air interface, which is evidenced by the observed birefringent smectic texture (see Figure 4.8b).
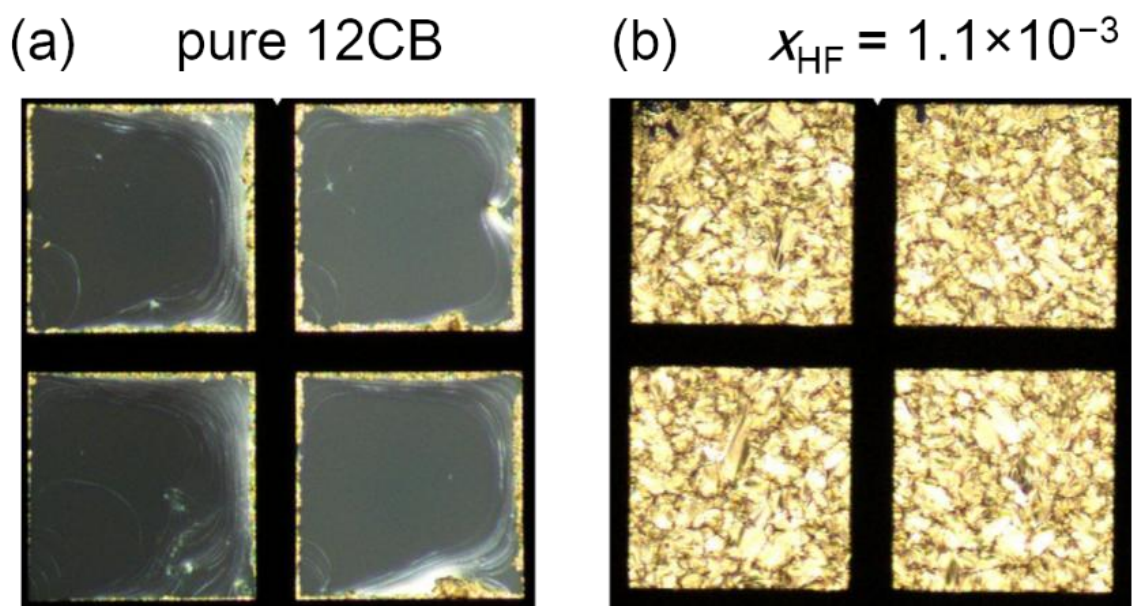

Figure 4.8 Polarized light microscopic images of $20 \mu \mathrm{m}$ thick films of pure 12CB (a) and of the 12CB sample with $x_{\mathrm{HF}}=1.1 \times 10^{-3}$ (b) at a temperature $1 \mathrm{~K}$ below the bulk smectic-isotropic transition. The films are prepared on a DMOAP-coated glass slide by filling a TEM grid (lateral width of the grid holes is $420 \mu \mathrm{m}$ ). Whereas pure 12CB shows a homeotropic anchoring at the air interface, the birefringent smectic texture of the H18F12-doped 12CB sample indicates a planar anchoring at the air interface. 
(a) $\quad T-T_{\mathrm{b}}=-0.5 \mathrm{~K}$

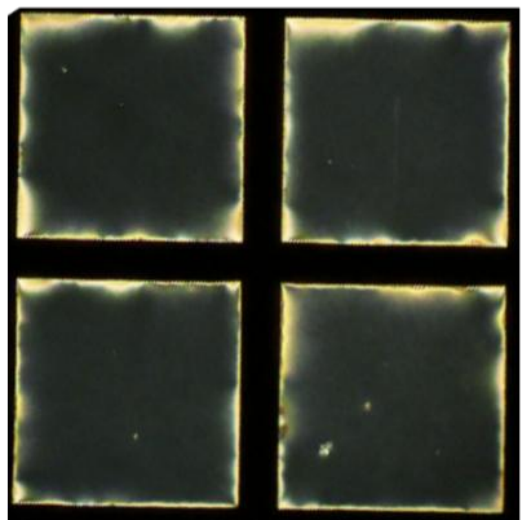

(b) $T-T_{\mathrm{b}}=-4 \mathrm{~K}$

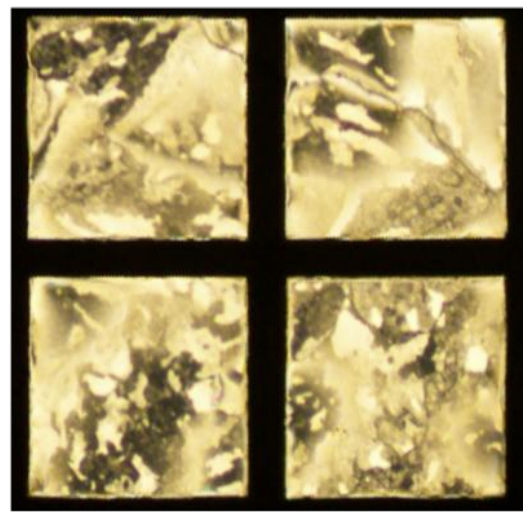

Figure 4.9 Polarized light microscopic images of $20 \mu \mathrm{m}$ thick films of an H18F12-doped 8CB sample with $x_{\mathrm{HF}}=5.7 \times 10^{-5}$ at (a) a temperature $0.5 \mathrm{~K}$ and (b) a temperature $4 \mathrm{~K}$ below the bulk nematic-isotropic transition. The films are prepared on a DMOAP-coated glass slide by filling a TEM grid (lateral width of the grid holes is $420 \mu \mathrm{m}$ ). Whereas the dilute Gibbs film induces a homeotropic anchoring at the air interface, the formation of the condensed Gibbs film causes an anchoring transition of the liquid crystal.

In addition, by characterizing temperature-dependent textures of a nematic film of $8 \mathrm{CB}$ with one surface laden with H18F12 molecules, the different effects exerted by the dilute state and the condensed state of the Gibbs film on the anchoring behavior can be distinguished. Here, the 8CB sample used for forming the nematic film is with a mole fraction of H18F12 of $5.7 \times 10^{-5}$. The ellipsometric data shown above indicates a structural phase transition of the Gibbs film in the nematic temperature range of $8 \mathrm{CB}$ (see Figure 4.5). Figure 4.9a displays a polarized light microscopic image of the nematic $8 \mathrm{CB}$ film when $T-T_{b}=-0.5 \mathrm{~K}$. Since at this temperature the Gibbs film is still in the dilute state, the nematic film is homeotropically anchored by the two surfaces. Figure $4.9 \mathrm{~b}$ shows the texture of the nematic $8 \mathrm{CB}$ film when $T-T_{b}=-4 \mathrm{~K}$ and the Gibbs film is in the condensed state. The detected transmission through the crossed polarizer demonstrates that the director in the film is not oriented perpendicular to the two surfaces. Such an anchoring transition is caused by the structural phase transition of the Gibbs film at the air interface. Thus, the polarized light microscopic study further confirms the surface anchoring from the Gibbs film, depending on its structures, can be homeotropic (for the dilute state) or planar (for the condensed state). 


\subsection{Conclusions}

The influence of Gibbs films formed by SFA molecules on the surface ordering and anchoring behavior at air interfaces of thermotropic liquid crystals have been studied. Investigations using ellipsometry and polarized light microscopy have revealed that the structural phase transition of Gibbs films of H18F12 molecule changes the nature of the surface field at air interfaces of liquid crystals. Whereas the dilute state of Gibbs films just reduces the strength of the homeotropic anchoring at air interfaces, the formation of condensed Gibbs films causes an anchoring transition from homeotropic to planar. The observed behavior, if compared with previous studies in which Gibbs films of surfactant molecules formed at liquid crystal/water interfaces, seems to be rather surprising. It has been known that the presence of surfactant molecules changes the anchoring effect of water from planar to homeotropic and an increase of surfactant coverage results in a stronger effect of homeotropic anchoring [41-43]. Nevertheless, Gibbs films of H18F12 molecules exert opposite effects on the anchoring at air interfaces by reducing or even destroying the homeotropic anchoring. This uncommon behavior may be due to the fact that the strength of the homeotropic anchoring of the air interface is so strong (although the mechanism is unknown so far) that any surface modification leads to a reduction of the strength of the homeotropic anchoring. Furthermore, the planar anchoring induced by condensed Gibbs films is quite unique. It is generally assumed that the aligning effect of the surfactant layer is based on a partial penetration of the liquid crystal molecules into the surfactant layer where the liquid crystal molecules orient along the nonpolar tails of the surfactant molecules. Although theoretical studies have predicted that the strength and type of anchoring change in a nonmonotonic way with the surface coverage of the surfactant layer and that planar anchoring at a dense surfactant layer can occur [135-136], it is difficult to experimentally demonstrate these predictions in a conventional system of surfactant-laden liquid crystal/water interfaces. As discussed in Chapter 3, once the surfactant concentration in the bulk solution reaches the CMC, the surface coverage is at the maximum but may be still not dense enough to prevent penetration of the liquid crystal molecules. There are some cases in which dense LB films of surfactant molecules at solid substrates [59] or dense monolayers of fatty acids at nematic/water interfaces [40] have been found to induce rather week homeotropic or non-homeotropic anchoring, but the creation of these systems requires many experimental efforts. Thus, liquid crystal/air interfaces 
laden with condensed Gibbs films provides an easy way to test the anchoring of liquid crystals in contact with dense alkyl chains

This study has shown for the first time that the ordering and anchoring behavior at liquid crystal/air interfaces can be influenced by adsorption of SFA molecules. The results may be of practical interests for future studies of freely suspended smectic films which are usually studied in air. An earlier report has concerned the study of the freely suspended smectic films in aqueous environment where the surface properties of the films could be modified by adsorption of surfactant molecules [137]. However, such smectic films are difficult to prepare in an aqueous solution and extremely susceptible to any perturbation such as flow of water. Thus, the use of SFA molecules to modify the surface property of smectic films in air environment can simplify the experimental procedures. Finally, the previously observed rich surface phase transitions such as prewetting transitions and novel smectic layering transitions in the system of surfactant-laden liquid crystal interfaces [41-43] have not been achieved in this study. Thus, it is necessary to further investigate other SFA molecules with different chain lengths of the perfluoroalkyl and the alkyl blocks because their surface-active properties strongly depend on the molecular architectures of SFA molecules [80-81]. Preliminary studies have indicated that it is possible to obtain Gibbs films with a continuous variation between dense and dilute states by using other SFAs, leading possibly to a richer variety in the liquid crystal surface ordering behavior. 


\section{Chapter 5. AFM Visualization of Gibbs Films of Semifluorinated Alkanes at Liquid Crystal/Air Interfaces}

\subsection{Introduction}

Structure and phase transitions of molecular layers formed by amphiphiles or surfactants at liquid interfaces have been of on-going interest [71-73]. As discussed in Chapter 1, there are two types of molecular layers at liquid interfaces, the forming processes of which are different: Langmuir films are formed by spreading of insoluble molecules and Gibbs films by adsorption of soluble molecules. For both types of molecular layers, structural phase transitions from a lesscondensed state to a more condensed state can occur under certain thermodynamic conditions [71-73]. The thermodynamic properties of the structural phase transitions are studied by using surface pressure-area isotherms (for Langmuir films) or temperature dependence of surface tension measurements (for Gibbs films). Moreover, investigations of the molecular ordering in Langmuir films or Gibbs films, particularly in the condensed states, provide insight into the main characteristics of these films. The molecular ordering in condensed Langmuir films or Gibbs films may result in regular textures and structures consisting of molecular aggregates. Whereas $\mathrm{X}$-ray diffraction and reflectivity as well as neutron scattering/reflectivity can resolve the ordered structure on the molecular scale [73], Brewster angle microscopy are often employed to visualize the morphology of ordered domains with sizes of micrometer [72]. The application of these techniques has confirmed a variety of ordered structures formed in the condensed phase of Langmuir films or Gibbs films. However, structuring on a mesoscopic length scale, in a range from $\approx 5 \mathrm{~nm}$ to $\approx 500 \mathrm{~nm}$, may be difficult to be detected by the above-mentioned techniques. The technique of AFM is capable of resolving surface structures on the scale of nanometer or micrometer. For Langmuir films, in situ studies are usually difficult to conduct since condensed Langmuir films are formed by using an LB trough (with lateral size of tens of centimeters) to compress monolayers of molecules on the surface of a given subphase (water). There have been some attempts to integrate the technique of AFM into LB troughs [138-139], but for the most cases Langmuir films are transferred onto solid substrates before they are studied by AFM. Since 
Gibbs films are formed by adsorption of molecules onto liquid interfaces and are in equilibrium with the bulk phase, the size of interfacial areas is without restriction. This advantage gives a hint that Gibbs films, when formed at surfaces of tiny droplets, are suitable for AFM characterizations [140].

Previous studies by surface tension measurements and surface-sensitive X-ray techniques have confirmed that Gibbs films of H18F12 formed at surfaces of hydrocarbon solutions exhibit sharp first-order phase transitions from a gas-like state at high temperatures to a condensed state at lower temperatures [47]. In Chapter 4, the influence of the structural phase transitions in Gibbs films on the surface ordering and anchoring behavior of thermotropic liquid crystals is already described. However, ellipsometry cannot answer the question how the molecular ordering in the condensed Gibbs films is. Grazing incidence X-ray scattering (GIXS) and X-ray reflectivity have revealed results which are difficult to interpret: Within the film plane, a dense hexagonal packing of the molecules but a short positional correlation length of $2 \mathrm{~nm}$ was found [47]. Perpendicular to the film plane, the electron density profile indicated that the mass centers of the molecules are not aligned in a plane but distributed over a region of 2-3 nm width. Furthermore, AFM studies of transferred Langmuir films and spin-coated or dipped coated films of SFA molecules on solid substrates have shown that these molecules are able to form self-assembled surface domains of large sizes (about 30-60 nm) [79, 141-143]. The observed surface domains can be considered as hemi-micelles in which the fluorinated alkyl chains of the SFA molecules point to the air and the alkyl chains are in contact with the substrate. However, whether such a self-organized behavior can be observed in condensed Gibbs films is still an open question. In addition, the structural information from condensed Gibbs films may help to explain why Gibbs films in such a state exerts an effect of planar anchoring to the liquid crystals in contact (see Chapter 4).

In order to resolve the possible superstructures formed in condensed Gibbs films of H18F12 molecules, tapping-mode AFM is employed to visualize the surface of liquid crystals at which the Gibbs films are in the condensed state. The principle of operating the tapping-mode AFM and the detailed experimental procedures are already described in Chapter 2. In a typical experiment, the surface of a tiny liquid crystal droplet (of several millimeters in diameter) containing an appropriate amount of $\mathrm{H} 18 \mathrm{~F} 12$ that is placed on a DMOAP-substrate is scanned by an instrument of AFM. The temperature is controlled so that the liquid crystal is in the smectic 
phase and the Gibbs film is in the condensed state. Topographic images of the Gibbs film at the surface of the smectic liquid crystal are thus obtained. In addition, although the shape of the macroscopic liquid crystal droplet appears to be constant, at the foot of the droplet a precursor film consisting of both liquid crystal molecules and H18F12 molecules may develop. Thus, it is also interesting to use AFM to characterize the precursor film, which might provide useful information about the diffusion of H18F12 molecules.

\subsection{Results and Discussion}

\subsubsection{AFM Measurements on Surfaces of Liquid Crystal Droplets Laden with SFA Molecules}

Figure 5.1a and $1 \mathrm{~b}$ show the topographic images of the surfaces of $12 \mathrm{CB}$ and $8 \mathrm{CB}$ droplets with the H18F12 mole fractions of $x_{\mathrm{HF}}=1.1 \times 10^{-3}(12 \mathrm{CB})$ and $x_{\mathrm{HF}}=6.0 \times 10^{-5}(8 \mathrm{CB})$, respectively. And the temperatures at which the AFM measurements were conducted were $55^{\circ} \mathrm{C}(12 \mathrm{CB})$ and $22^{\circ} \mathrm{C}(8 \mathrm{CB})$, respectively. The ellipsometric study presented in Chapter 4 has already confirmed that at both temperatures the Gibbs films are in the condensed state. The AFM height images shown in Figure 5.1 demonstrate that in both cases the condensed Gibbs films consist of closepacked hexagonal domains which are in analogy with the so-called surface micelles observed in transferred Langmuir films and spin-coated or dipped coated films of SFA molecules on solid substrates [79, 141-143]. A Fourier transformation (see Figure 5.1c) of the height image of 12CB illustrates a well-defined hexagonal lattice with only a small deformation: From the three pairs of first-order peaks, a periodicity of $33 \mathrm{~nm}$ along two in-plane directions and $37 \mathrm{~nm}$ along the third direction is obtained for the condensed Gibbs film on the 12CB surface. Similarly, the corresponding values for the condensed Gibbs film on the $8 \mathrm{CB}$ surface are $30 \mathrm{~nm}$ and $36 \mathrm{~nm}$, respectively. The size of surface micelles formed in Langmuir films of SFA molecules are mainly influenced by the lengths of the alkyl chain [143]. The data obtained here for H18F12 can be compared with those for Langmuir films which show a value of $34 \mathrm{~nm}$ (or $28 \mathrm{~nm}$ as accessed by a different software) for the average diameter of H18F8 micelles [143]. Therefore, the size of the surface micelles found in the condensed Gibbs films at liquid crystal surfaces is in good 
(a)

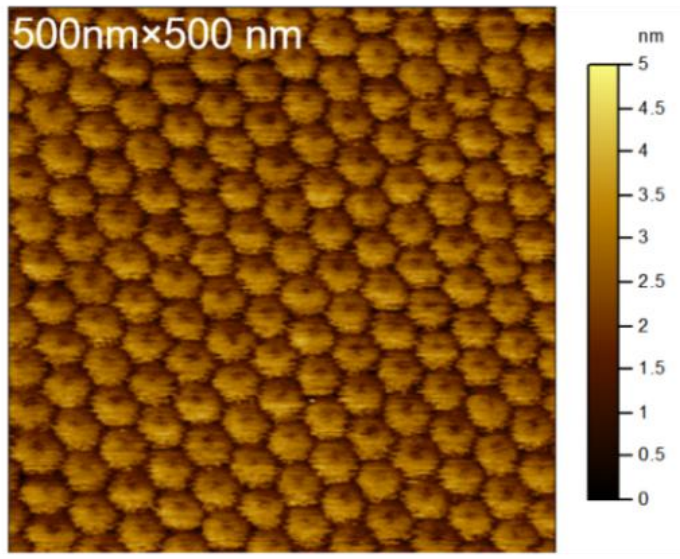

(c)

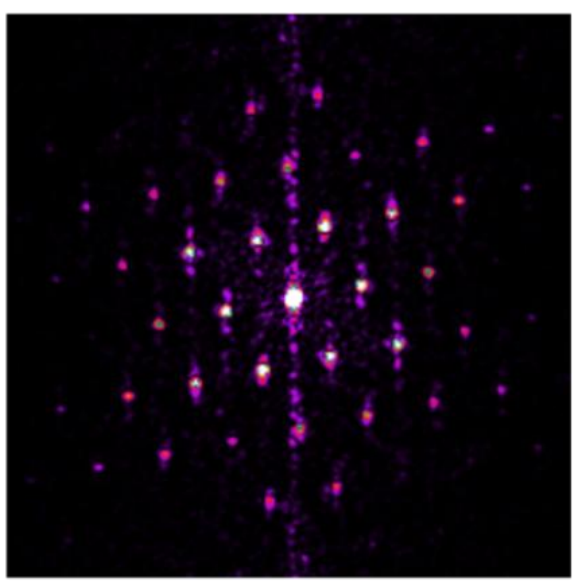

(b)

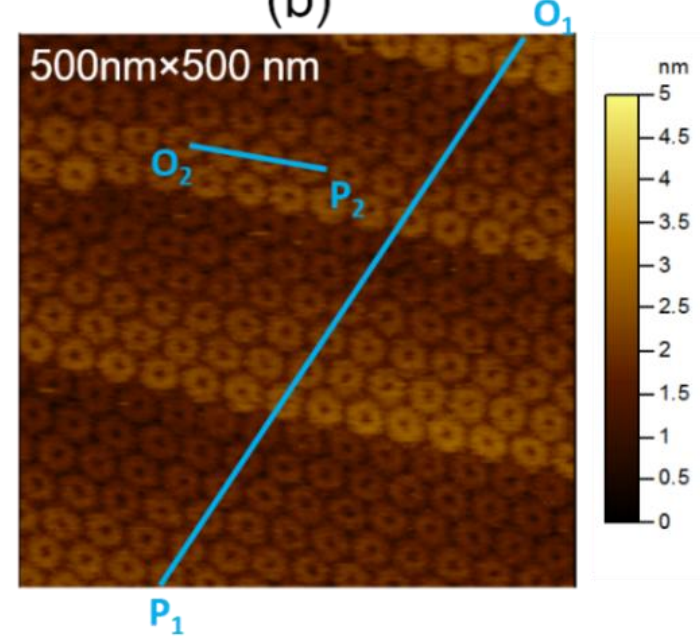

(d)

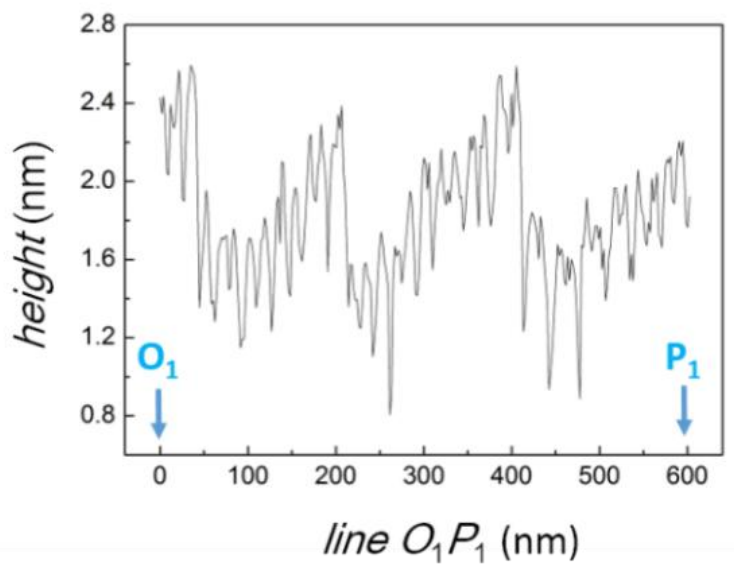

(e)

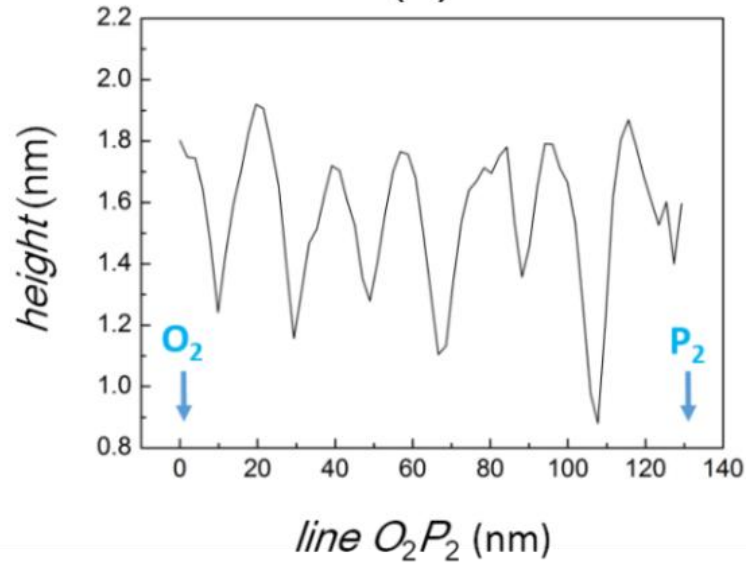

Figure 5.1 Results obtained from AFM measurements on $12 \mathrm{CB}$ and $8 \mathrm{CB}$ surfaces laden with H18F12 molecules. The H18F12 mole fractions in $12 \mathrm{CB}$ and $8 \mathrm{CB}$ are $x_{\mathrm{HF}}=1.1 \times 10^{-3}$ and $x_{\mathrm{HF}}=6.0 \times 10^{-5}$, respectively. Parts (a) and (b) are height images of $12 \mathrm{CB}$ and $8 \mathrm{CB}$; part (c) shows a Fourier transformation of part (a); parts (d) and (e) correspond to cross sections through the lines $O_{1} P_{1}$ and $O_{2} P_{2}$ in part (b). 
agreement with the reported data for Langmuir films. Here, the AFM topographic images reveal a special feature of the Gibbs films at the liquid crystal surfaces. The shape of each surface domain appears to be hexagonal and their packing forms a two-dimensional crystalline structure. However, surface micelles observed in Langmuir films are often circular or elongated and their packing is not so regular [143]. Sometimes organized and stable hexagonal networks of surface micelles can be obtained only when the Langmuir films are transferred at a high surface pressure [79]. A previous report has shown that spin-coated films of SFA molecules on water-wet substrates can form highly organized hexagonal surface micelles [141].

At the surfaces of the smectic liquid crystals under investigation, sometimes the Gibbs films are found to contain some terraced superstructures, as shown in Figure 5.1b for an $8 \mathrm{CB}$ sample. A cross sectional analysis of these steps shown in Figure 5.1d reveals that their height is roughly $1 \mathrm{~nm}$. And the lateral distance of these steps is found to be not constant (although Figure 5.2b shows a correlation of about $200 \mathrm{~nm}$, measurements on different regions of the Gibbs film yield different values). This behavior can be attributed to the nature of smectic liquid crystals which contain molecular layers in the bulk. It has been known that smectic droplets on solid substrates can show a terraced structure when the molecular layers lie flat on the substrate, with the height steps on the surface then corresponding to the thickness of the molecular smectic layers (typically 3-4 nm for $\mathrm{nCB}$ compounds) [144]. It is obvious that the height of the steps observed here is much smaller than the thickness of a smectic layer of $8 \mathrm{CB}$. Moreover, a simple terraced structure, consisting of a stack of plane layers, can only be expected for perpendicular (homeotropic) anchoring on both interfaces (substrate and air). However, the silicon substrates used here are coated by DMOAP, which induces strong homeotropic anchoring. But at the air interface, which is covered by the condensed H18F12 Gibbs film, the anchoring is found to be planar (see Chapter 4). Thus, the two surfaces of the smectic drop induce different anchoring effects which lead to a bend deformation of the smectic layers. It might be difficult to clarify the arrangement of the smectic layers under this situation, but the bend deformation of the smectic layers can be assumed to account for the formation of the step-like structure of the surface.

To get rid of the observed step-like structure, one can use nematic liquid crystals or isotropic liquids such as simple alkanes like hexadecane to form droplets for the AFM study. For instance, the condensed Gibbs film may be characterized by AFM at an elevated temperature which allows 
$8 \mathrm{CB}$ to be in the nematic state. However, it was found that nematic or isotropic samples showed a strong tendency to form a meniscus between the AFM tip and the sample, which makes it difficult to conduct AFM measurements on such surfaces.

The results obtained by AFM measurements demonstrate that condensed Gibbs films of H18F12 are not featureless but consist of regular hexagonal surface micelles which are in analogy with those formed in Langmuir films or spin-coated films. By a careful examination of the AFM images, one observes that these surface micelles have a tiny dip at their centers. A cross sectional analysis of these surface micelles, as presented in Figure 5.1e, shows a depth of about $0.5-0.6 \mathrm{~nm}$. However, it should be noted that the resolution of the AFM is limited by the shape of the tip. A disc-like model, in which the SFA molecules lie nearly flat along the edge of the surface micelles, has been proposed to interpret the formation of the surface micelles in Langmuir films $[79,143]$. In view of the fact that similar surface micelles are also formed in the condensed Gibbs films, this model can be used here to describe the molecular organization at the liquid crystal surfaces. This model might further explain the observation that the dense Gibbs film of H18F12 induces random planar anchoring of the liquid crystal in contact with it (see Chapter 4). The presence of the surface micelles could also explain the x-ray results obtained in [47]: Since the H18F12 molecules are located within the surface micelles and at the edge of the micelles in different planes, a broadening in the electron density profile obtained from x-ray reflectivity can be expected. Also the short lateral correlation length obtained from the GIXS measurements can be expected, because the long-range positional correlation of the H1812 molecules is interrupted by the micelle edges and by the hole at the center of the micelles.

\subsubsection{AFM Measurements on the Precursor Film Spreading from a 12CB Droplet Doped with H18F12 Molecules}

The shape of the droplets on the substrates under investigation by AFM remained constant for days because the substrates were coated by the silane DMOAP which increases the contact angle of the liquid crystals. This condition is well suited for AFM scanning of the surfaces of the droplets. However, by optical observation, a precursor film was found to develop from the foot the droplet, up to centimeter extensions. The characterization of the precursor film by AFM provides insights into the response of the condensed Gibbs film to a non-equilibrium situation: 
The spreading of the precursor film considerably increases the area of the droplet-film system while it prevents a sufficient diffusion of SFA molecules from the bulk droplet to maintain a dense layer of surface micelles on the precursor film. Thus, one would expect that the density of the surface micelles in the precursor film decreases with increasing the distance $d$ from the foot of the droplet.

(a) $\quad d=5 \mu \mathrm{m}$

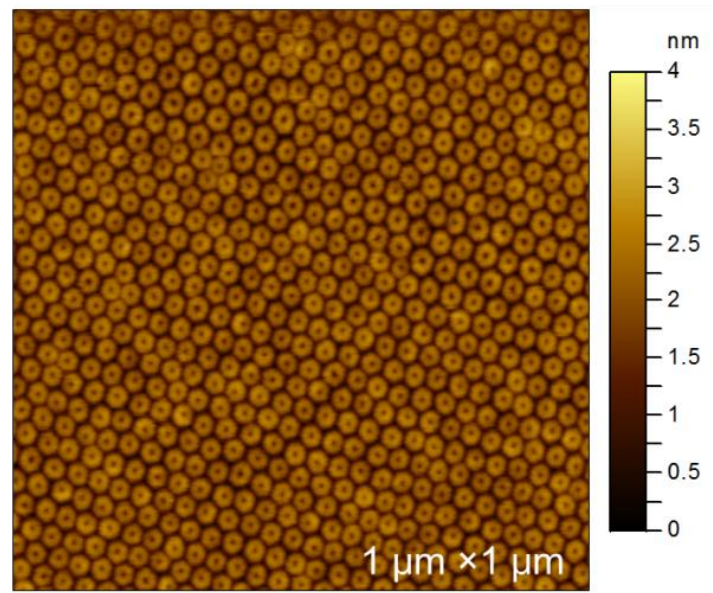

(c)

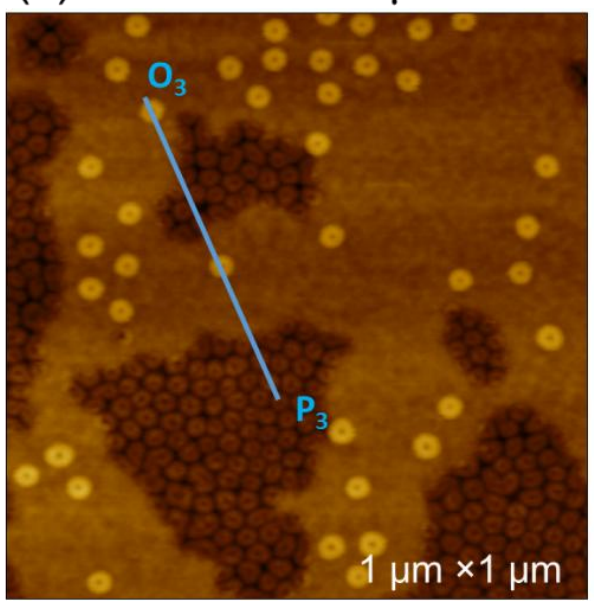

(b) $\quad d=60 \mu \mathrm{m}$

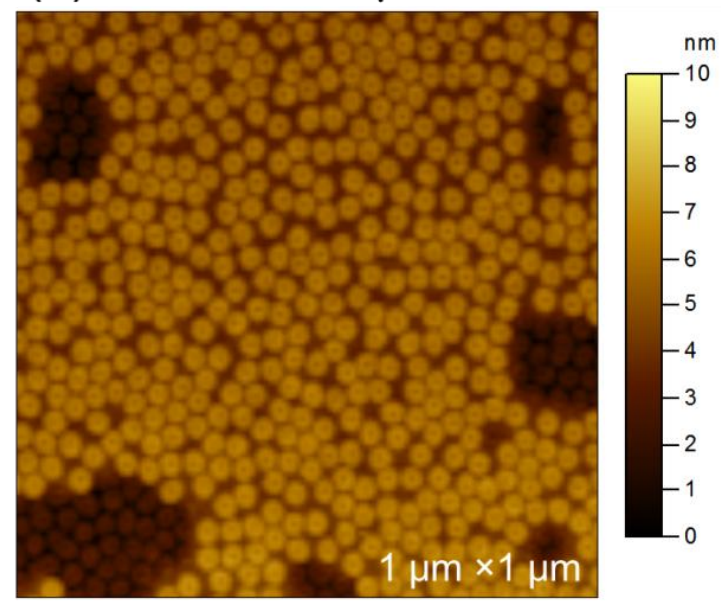

(d)

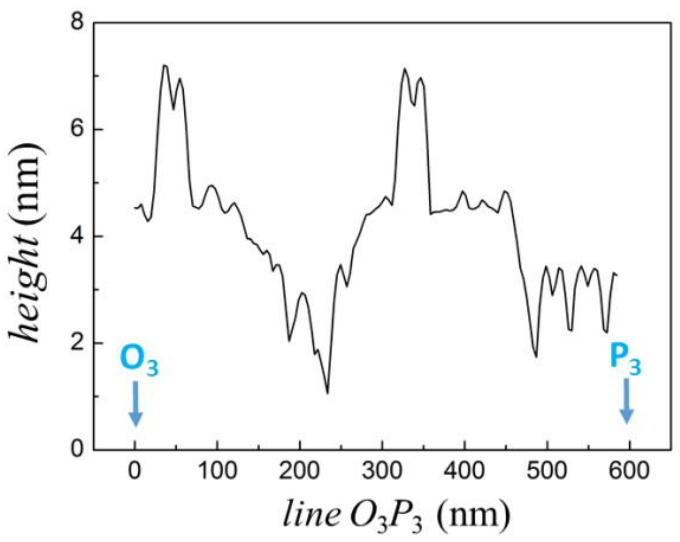

Figure 5.2 AFM images of the surface of a precursor film spreading from a 12CB droplet doped with H18F12 $\left(x_{\mathrm{HF}}=1.1 \times 10^{-3}\right)$. The AFM measurements were conducted at different regions with different distances $d$ from the foot of the droplet. The value of $d$ is indicated in each image. Part (d) shows the cross section along the line $\mathrm{O}_{3} P_{3}$ in (c). 
Figure 5.2a-c shows the AFM topographic images of the surface of the precursor film with different distances from the foot of the static macroscopic 12CB droplet. The precursor film was allowed to spread for about $24 \mathrm{~h}$ at $55{ }^{\circ} \mathrm{C}$ before the AFM measurements. The surface micelles are also observable in the precursor film and the morphology is consistent with those found in the Gibbs film or Langmuir films. At a region very close to the edge of the macroscopic 12CB droplet (e.g., $d=5 \mu \mathrm{m}$, see Figure 5.1a), the surface micelles have the same hexagonal shape as observed in the condensed Gibbs film at the surface of the microscopic droplet. The density and the ordered packing of surface micelles in this region are similar to those in the condensed Gibbs films. An increase of the value of $d$ results in the expected decrease of the micelle density, as demonstrated in Figure 5.2b for $d=60 \mu \mathrm{m}$. In addition, the shape of the surface micelles in this region appears to be circular and the two-dimensional crystalline order is not present any more.

An unexpected feature observed in Figure 5.2b is the occurrence of "big holes": There are some small discontinuous regions the height of which is smaller than the major area. Surface micelles are also existent within these "big holes". For a still larger distance from the droplet edge ( $d=100 \mu \mathrm{m}$, see Figure 5.2c), the area of "holes" has increased. In the "big holes", the coverage of surface micelles is rather dense whereas on the surface of the liquid crystal precursor film only isolated surface micelles are present. A cross section of the height image (see Figure $5.2 \mathrm{~d}$ ) shows that the height of the isolated micelles is about $3 \mathrm{~nm}$. The height difference between the apex of the micelles on top of the precursor film and the apex of the micelles in the holes of the precursor film is about $4 \mathrm{~nm}$ which coincides well with the thickness of a single smectic layer of $12 \mathrm{CB}$. These findings imply that the isolated surface micelles are located on top of a smectic layer whereas the surface micelles within the "holes" directly sit on the solid substrate. Remarkably, for $d>110 \mu \mathrm{m}$, both the isolated surface micelles and the holes filled with the dense micelle layer vanish completely whereas a homogeneous liquid crystal precursor film still extends over distances of several millimeters. It seems that the condensed H18F12 film on the surface of the spreading liquid crystal precursor film undergoes two pathways: The first is the expected density decrease, the hexagonal arrangement of the surface micelles changes first to a liquid-like and then to a gas-like arrangement. The second is a kind of lateral segregation process, the liquid crystal precursor film with the dense H18F12 layer on the surface separates into regions where a precursor film with a less dense H18F12 film exists, and regions in which a 
dense H18F12 film is located directly on the substrate and a liquid crystal precursor film is not present. Additional studies are needed to clarify the reasons for this behavior.

\subsection{Conclusions}

Condensed Gibbs films of SFA molecules at surfaces of smectic liquid crystal droplets have been in situ characterized by AFM. This study has demonstrated that the technique of AFM is a well suited one for observing surface structures with sizes of ten-to-hundred nanometers formed in Gibbs films. Thus, the technique of AFM can fill the observation gap between Brewster angle microscopy and surface-sensitive X-ray and neutron techniques. The condensed Gibbs films of $\mathrm{H} 18 \mathrm{~F} 12$ molecules have been found to form hexagonal surface micelles at surfaces of $12 \mathrm{CB}$ or $8 \mathrm{CB}$ in the smectic phase. The packing of the hexagonal surface micelles displays a twodimensional crystalline structure. In addition, similar surface micelles can be also observed in the spreading precursor film at the foot of a liquid crystal droplet covered by a condensed Gibbs film. The observations in this study, together with previous studies of Langmuir films and spin-coated films of SFA molecules, demonstrate that the ability to form surface micelles in thin films is an intrinsic property of SFA molecules. Furthermore, the observation of surface micelles provides supplementary information to elucidate the ordered structure in condensed Gibbs films of H18F12 which was first studied by GIXS and X-ray reflectivity. Finally, the molecular orientation in the disc-like surface micelles may be responsible for the effect of planar anchoring exerted on the liquid crystals in contact with the condensed Gibbs films. 


\section{Chapter 6. Summary and Outlook}

In this dissertation, a study of the structures and self-organized behaviors at liquid crystal interfaces laden with surface-active molecules has been presented. The interfaces under investigation here are the ones formed between liquid crystals and fluids or between liquid crystals and air, rather than the well-established liquid crystal/solid interfaces. One specific feature of these liquid crystal interfaces is that they are laden with an adsorbed layer of surfaceactive molecules which is in equilibrium with the bulk phase. Such liquid crystal interfaces have been proved to be ideal model systems for the study of surface phase transitions, surface-induced order, surface layering, and wetting. By using ellipsometry, polarized light microscopy and atomic force microscopy, the experimental work has been done to explore the ordering processes and structures occurring at liquid crystal interfaces.

First, the pretransitional liquid crystal order at interfaces of isotropic liquid crystals has been investigated with respect to the influence of the properties of surface-active species such as surfactants or other amphiphiles. The interfacial behavior of the surface-active molecules has been tuned by tuning the bulk properties in the volume phase. Two experimental systems have been designed here to investigate the influence of surface-active molecules on the pretransitional liquid crystal order.

One experimental system concerns the interface between thermotropic liquid crystals and liquid phases consisting of various binary mixtures of water and glycerol. The liquid-crystal samples contain a small constant amount of a surfactant which induces a homeotropic anchoring at the interface. By varying the volume fraction of glycerol in the water/glycerol phase which leads to a systematic variation of the polarity of the immiscible phase in contact with the isotropic liquid crystal phase, the surface coverage of the surfactant molecules at the interface can be varied correspondingly. It is found that an increase of the polarity of the immiscible phase, which leads to a decrease of the surface coverage of the surfactant molecules, can allow for the observations of nematic prewetting transitions and smectic multiple-layering transitions. Thus, this finding together with the previous reports shows that a higher surface coverage of surface- 
active molecules, corresponding to a stronger strength of homeotropic anchoring, can enhance the surface-induced pretransitional order at liquid crystal interfaces.

Another experimental system is the liquid crystal/air interface laden with semifluorinated alkane molecules. For the first time, the pretransitional liquid crystal order at liquid crystal/air interfaces has been manipulated by the adsorption of a special kind of surface-active molecules. The presence of the absorbed monolayer of semifluorinated alkane molecules can change the nature of the surface field at the air interfaces of liquid crystals. The influence of the semifluorinated alkane molecules on the pretransitional liquid crystalline order turns out to be different from those observed for common surfactant molecules. One effect consists of an increase of the surface coverage of the semifluorinated alkane molecules decreases the homeotropic anchoring strength of the air interface and thus suppresses the surface order; another feature is the absorbed monolayer of the semifluorinated alkane molecules undergoes a first-order structural phase transition into a condensed state at lower temperatures, which completely changes the behavior of the pretransitional liquid crystal order by destroying the smectic surface order or changing the orientation of the nematic surface order from homeotropic to planar. The observed behavior is rather surprising, since it has been believed that long alkyl chains in contact with liquid crystals usually promote the effect of homeotropic anchoring. In addition, a previous report has shown that semifluorinated alkanes can induce the surface freezing of an alkane which is unable to show such behavior alone.

Second, the surface anchoring behavior of liquid crystal films at the air interface has been manipulated by the adsorption of the semifluorinated alkane. The absorbed monolayer of the semifluorinated alkane molecules, depending on the surface coverage of the semifluorinated alkane molecules, can induce homeotropic (for the dilute state) or planar (for the condensed state) anchoring to the liquid crystals. The observed planar anchoring of the cyanobiphenyl liquid crystals in contact with the condensed monolayers of the semifluorinated alkanes is also of significant interest, since for a long time the intrinsic anchoring effect from the air interface had been considered unchangeable.

Finally, in situ AFM studies have been conducted to probe the structure and self-organized behavior of the absorbed monolayer of semifluorinated alkane molecules at the surface of liquid crystal droplets. The AFM measurements have revealed that the condensed monolayer of semifluorinated alkane molecules forms hexagonal surface micelles and their packing displays a 
two-dimensional crystalline structure at surfaces of smectic liquid crystal droplets. The selforganization of the surface micelles provides supplementary information to elucidate the ordered structure which was first investigated by surface-sensitive $\mathrm{X}$-ray techniques. The AFM measurements may also reveal that the uncommon effect of planar anchoring exerted on the liquid crystals is due to the molecular orientation in the disc-like surface micelles.

The study presented here demonstrates that the concept of surface-induced pretransitional order and surface anchoring of liquid crystals can be extended from the previously studied water interfaces to other soft interfaces. Thus, the results are of practical interests for future studies of liquid crystal interfacial phenomena. For example, emulsions of nematic droplets can be prepared in different liquid phases in addition to water. The possible use of a variety of liquids enables a variation of the bulk properties of the liquid phase such as refractive index and viscosity. The director configuration of the droplets could be controlled by the adsorption of surface-active molecules. If the adsorbed molecular layer of surface-active molecules could undergone similar structural phase transitions which cause the corresponding anchoring transitions, the director configuration of the droplets is switchable, which will be of interest in recent photonic applications of nematic droplets. Furthermore, the finding that the free surfaces of liquid crystals can be modified by semifluorinated alkanes is fascinating. Since it had been considered for a long time that the interfacial properties of the free interface of for a given liquid crystal cannot be manipulated, many potential studies which are related to the free surface of liquid crystals may have been ignored. The available examples are liquid crystal bubbles and free standing films. Furthermore, apart from semifluorinated alkanes, more semifluorinated compounds with adapted molecular structures which are more appropriate for future research could be designed. Finally, the successful utilization of AFM to visualize adsorbed monolayers at liquid crystal surfaces demonstrates that AFM is powerful technique for studying ordered structures at liquid surfaces. Unlike surface-sensitive X-ray and neutron techniques, it is easy to set up and operate in a lab. 


\section{References}

[1] C. A. Croxton, Fluid interfacial phenomena, Wiley, New York, 1986.

[2] K. B. Eisenthal, Accounts of Chemical Research 1993, 26, 636-643.

[3] J. Penfold, Reports on Progress in Physics 2001, 64, 777.

[4] P. Lang, Journal of Physics: Condensed Matter 2004, 16, R699.

[5] D. Bonn, J. Eggers, J. Indekeu, J. Meunier, E. Rolley, Reviews of Modern Physics 2009, 81, 739.

[6] X. Z. Wu, E. B. Sirota, S. K. Sinha, B. M. Ocko, M. Deutsch, Physical Review Letters 1993, 70, 958-961.

[7] B. M. Ocko, X. Z. Wu, E. B. Sirota, S. K. Sinha, O. Gang, M. Deutsch, Physical Review E 1997, $55,3164-3182$.

[8] O. M. Magnussen, B. M. Ocko, M. J. Regan, K. Penanen, P. S. Pershan, M. Deutsch, Physical Review Letters 1995, 74, 4444-4447.

[9] E. Sloutskin, B. M. Ocko, L. Tamam, I. Kuzmenko, T. Gog, M. Deutsch, Journal of the American Chemical Society 2005, 127, 7796-7804.

[10] K. Miyano, Physical Review Letters 1979, 43, 51-54.

[11] B. M. Ocko, A. Braslau, P. S. Pershan, J. Alsnielsen, M. Deutsch, Physical Review Letters 1986, 57, 94-97.

[12] B. M. Ocko, Physical Review Letters 1990, 64, 2160-2163.

[13] B. Jerome, Reports on Progress in Physics 1991, 54, 391.

[14] S. Chandrasekhar, Liquid crystals, Cambridge University Press, Cambridge, 1992.

[15] P. G. de Gennes, J. Prost, The Physics of Liquid Crystals, Oxford University Press, London, 1994.

[16] W. H. de Jeu, Physical Properties Of Liquid Crystalline Materials Taylor \& Francis Group, 1980.

[17] S. Singh, D. A. Dunmur, Liquid Crystals: Fundamentals, World Scientific, 2002.

[18] L. M. Blinov, V. G. Chigrinov, Electrooptic Effects in Liquid Crystal Materials, Springer-Verlag, 1996.

[19] S. T. Wu, D. K. Yang, Fundamentals of Liquid Crystal Devices, Wiley, 2006.

[20] T. Rasing, I. Musevic, Surfaces and Interfaces of Liquid Crystals, Springer, 2004.

[21] K. Takatoh, Alignment Technology and Applications of Liquid Crystal Devices, CRC PressINC, 2005.

[22] I.-H. Lin, D. S. Miller, P. J. Bertics, C. J. Murphy, J. J. de Pablo, N. L. Abbott, Science 2011, 332, 1297-1300.

[23] T. Bera, J. Fang, Journal of Materials Chemistry 2012, 22, 6807-6812.

[24] S. Sivakumar, K. L. Wark, J. K. Gupta, N. L. Abbott, F. Caruso, Advanced Functional Materials 2009, 19, 2260-2265.

[25] M. Humar, M. Ravnik, S. Pajk, I. Musevic, Nature Photonics 2009, 3, 595-600.

[26] T. Lopez-Leon, V. Koning, K. B. S. Devaiah, V. Vitelli, A. Fernandez-Nieves, Nature Physics 2011, 7, 391-394.

[27] H.-L. Liang, R. Zentel, P. Rudquist, J. Lagerwall, Soft Matter 2012, 8, 5443-5450.

[28] K. Peddireddy, P. Kumar, S. Thutupalli, S. Herminghaus, C. Bahr, Langmuir 2012, 28, 1242612431.

[29] Y. Iwashita, C. Bahr, C. Priest, S. Herminghaus, R. Seemann, La Houille Blanche 2009, 129-134.

[30] V. G. Nazarenko, A. B. Nych, B. I. Lev, Physical Review Letters 2001, 87.

[31] A. B. Nych, U. M. Ognysta, V. M. Pergamenshchik, B. I. Lev, V. G. Nazarenko, I. Musevic, M. Skarabot, O. D. Lavrentovich, Physical Review Letters 2007, 98.

[32] J. M. Brake, N. L. Abbott, Langmuir 2002, 18, 6101-6109. 
[33] J. M. Brake, M. K. Daschner, Y. Y. Luk, N. L. Abbott, Science 2003, 302, 2094-2097.

[34] Y. Y. Luk, M. L. Tingey, K. A. Dickson, R. T. Raines, N. L. Abbott, Journal of the American Chemical Society 2004, 126, 9024-9032.

[35] N. A. Lockwood, J. K. Gupta, N. L. Abbott, Surface Science Reports 2008, 63, 255-293.

[36] A. D. Price, D. K. Schwartz, Journal of the American Chemical Society 2008, 130, 8188-8194.

[37] C. H. Jang, L. L. Cheng, C. W. Olsen, N. L. Abbott, Nano Letters 2006, 6, 1053-1058.

[38] A. D. Price, J. Ignes-Mullol, M. A. Vallve, T. E. Furtak, Y.-A. Lo, S. M. Malone, D. K. Schwartz, Soft Matter 2009, 5, 2252-2260.

[39] J. M. Brake, A. D. Mezera, N. L. Abbott, Langmuir 2003, 19, 8629-8637.

[40] A. D. Price, D. K. Schwartz, Journal of Physical Chemistry B 2007, 111, 1007-1015.

[41] C. Bahr, Physical Review E 2006, 73, 030702.

[42] C. Bahr, Physical Review Letters 2007, 99, 057801.

[43] C. Bahr, EPL (Europhysics Letters) 2009, 88, 46001.

[44] M. G. J. Gannon, T. E. Faber, Philosophical Magazine A 1978, 37, 117-135.

[45] S. Faetti, V. Palleschi, Physical Review A 1984, 30, 3241-3251.

[46] P. Sheng, Physical Review A 1982, 26, 1610-1617.

[47] P. Marczuk, P. Lang, G. H. Findenegg, S. K. Mehta, M. Möller, Langmuir 2002, 18, 6830-6838.

[48] S. Friberg, Lyotropic liquid crystals and the structure of biomembranes: a symposium based on the fifth International Liquid Crystal Conference : proceedings : Stockholm, Sweden, June 17-21, 1974, American Chemical Society, 1976.

[49] D. Dunmur, T. Sluckin, Soap, Science, and Flat-Screen TVs:A History of Liquid Crystals: A History of Liquid Crystals, OUP Oxford, 2010.

[50] S. Chandrasekhar, G. S. Ranganath, Reports on Progress in Physics 1990, 53, 57.

[51] I. Dierking, Textures of Liquid Crystals, Wiley-VCH Verlag, Weinhaim, 2006.

[52] P. J. Collings, M. Hird, Introduction to Liquid Crystals: Chemistry and Physics, CRC Press INC, 1997.

[53] R. T. Syvitski, E. E. Burnell, Journal of Magnetic Resonance 2000, 144, 58-73.

[54] H. Yoshida, Y. Nakajima, S. Kobinata, S. Maeda, Journal of the Physical Society of Japan 1981, 50, 3525-3528.

[55] L. L. Chapoy, D. B. Dupre, Journal of Chemical Physics 1979, 70, 2550-2553.

[56] W. S. Park, Journal of the Korean Physical Society 2000, 37, 331-334.

[57] C. Mauguin, Comptes Rendus de l'Académie des Sciences 1911, 156, 1246.

[58] F. J. Kahn, Applied Physics Letters 1973, 22, 386-388.

[59] K. Hiltrop, H. Stegemeyer, Berichte Der Bunsen-Gesellschaft-Physical Chemistry Chemical Physics 1981, 85, 582-588.

[60] K. Hiltrop, H. Stegemeyer, Berichte Der Bunsen-Gesellschaft-Physical Chemistry Chemical Physics 1978, 82, 884-889.

[61] W. Guo, AFM Studies of Smectic Liquid Crystals: Focal Conic Domains in Smectic Films, $\mathrm{PhD}$ thesis, Georg-August-Universität Göttingen (Göttingen), 2009.

[62] J. L. Janning, Applied Physics Letters 1972, 21, 173-174.

[63] P. Poulin, H. Stark, T. C. Lubensky, D. A. Weitz, Science 1997, 275, 1770-1773.

[64] S. J. Woltman, G. D. Jay, G. P. Crawford, Nature Materials 2007, 6, 929-938.

[65] S. Faetti, L. Fronzoni, Solid State Communications 1978, 25, 1087-1090.

[66] M. A. Bouchiat, D. Langevin-Cruchon, Physics Letters A 1971, 34, 331-332.

[67] G. G. Alexander, R. Cubitt, R. M. Dalgliesh, C. Kinane, R. M. Richardson, H. Zimmermann, Physical Chemistry Chemical Physics 2011, 13, 14786-14796.

[68] S. Faetti, Molecular Crystals and Liquid Crystals 1990, 179, 217-231.

[69] A. D. Rey, Langmuir 2004, 20, 11473-11479.

[70] R. Lucht, C. Bahr, G. Heppke, Physical Review E 2000, 62, 2324-2333.

[71] V. M. Kaganer, H. Möhwald, P. Dutta, Reviews of Modern Physics 1999, 71, 779-819.

[72] D. Vollhardt, V. B. Fainerman, Advances in Colloid and Interface Science 2010, 154, 1-19. 
[73] M. L. Schlossman, A. M. Tikhonov, in Annual Review of Physical Chemistry, Vol. 59, Annual Reviews, Palo Alto, 2008, pp. 153-177.

[74] E. Sloutskin, Z. Sapir, C. D. Bain, Q. Lei, K. M. Wilkinson, L. Tamam, M. Deutsch, B. M. Ocko, Physical Review Letters 2007, 99, 136102.

[75] S. V. Pingali, T. Takiue, G. Luo, A. M. Tikhonov, N. Ikeda, M. Aratono, M. L. Schlossman, The Journal of Physical Chemistry B 2004, 109, 1210-1225.

[76] M. P. Krafft, J. G. Riess, Chemical Reviews 2009, 109, 1714-1792.

[77] O. Gang, J. Ellmann, M. Moller, H. Kraack, E. B. Sirota, B. M. Ocko, M. Deutsch, Europhysics Letters 2000, 49, 761-767.

[78] L. Bardin, M.-C. Fauré, D. Limagne, C. Chevallard, O. Konovalov, E. J. M. Filipe, G. Waton, M. P. Krafft, M. Goldmann, P. Fontaine, Langmuir 2011, 27, 13497-13505.

[79] M. P. Krafft, Accounts of Chemical Research 2011, 45, 514-524.

[80] B. P. Binks, P. D. I. Fletcher, S. N. Kotsev, R. L. Thompson, Langmuir 1997, 13, 6669-6682.

[81] B. P. Binks, P. D. I. Fletcher, W. F. C. Sager, R. L. Thompson, Langmuir 1995, 11, 977-983.

[82] T. J. Sluckin, A. Poniewierski, Physical Review Letters 1985, 55, 2907-2910.

[83] A. K. Sen, D. E. Sullivan, Physical Review A 1987, 35, 1391-1403.

[84] J. B. Fournier, P. Galatola, Europhysics Letters 2005, 72, 403-409.

[85] Z. Pawlowska, T. J. Sluckin, G. F. Kventsel, Physical Review A 1988, 38, 5342-5351.

[86] A. M. Somoza, L. Mederos, D. E. Sullivan, Physical Review E 1995, 52, 5017-5027.

[87] T. Moses, Y. R. Shen, Physical Review Letters 1991, 67, 2033-2036.

[88] J. C. Tarczon, K. Miyano, Journal of Chemical Physics 1980, 73, 1994-1998.

[89] D. Beaglehole, Molecular Crystals and Liquid Crystals 1982, 89, 319-325.

[90] S. Immerschitt, T. Koch, W. Stille, G. Strobl, Journal of Chemical Physics 1992, 96, 6249-6256.

[91] H. Kasten, G. Strobl, Journal of Chemical Physics 1995, 103, 6768-6774.

[92] P. DeSchrijver, C. Glorieux, W. VanDael, J. Thoen, Liquid Crystals 1997, 23, 709-715.

[93] R. Lucht, C. Bahr, Physical Review Letters 1997, 78, 3487-3490.

[94] R. Lucht, C. Bahr, Physical Review Letters 1998, 80, 3783-3786.

[95] H. Hsiung, T. Rasing, Y. R. Shen, Physical Review Letters 1986, 57, 3065-3068.

[96] B. Alkhairalla, H. Allinson, N. Boden, S. D. Evans, J. R. Henderson, Physical Review E 1999, 59, 3033-3039.

[97] R. Lucht, P. Marczuk, C. Bahr, G. H. Findenegg, Physical Review E 2001, 63, 041704.

[98] S. Aya, Y. Sasaki, F. Araoka, K. Ema, K. Ishikawa, A. V. Emelyanenko, H. Takezoe, Physical Review Letters 2011, 106, 117801.

[99] X. Liu, D. W. Allender, D. Finotello, EPL (Europhysics Letters) 2002, 59, 848.

[100] K. Kočevar, I. Muševič, ChemPhysChem 2003, 4, 1049-1056.

[101] G. P. Crawford, R. J. OndrisCrawford, J. W. Doane, Physical Review E 1996, 53, 3647-3661.

[102] T. Jin, G. P. Crawford, R. J. Crawford, S. Zumer, D. Finotello, Physical Review Letters 2003, 90.

[103] P. Ziherl, M. Vilfan, N. Vrbancic-Kopac, S. Zumer, R. J. Ondris-Crawford, G. P. Crawford, Physical Review E 2000, 61, 2792-2798.

[104] W. Chen, L. Martinez-Miranda, H. Hsiung, Y. Shen, Physical Review Letters 1989, 62, 18601863.

[105] G. S. Iannacchione, J. T. Mang, S. Kumar, D. Finotello, Physical Review Letters 1994, 73, 27082711.

[106] B. Alkhairalla, N. Boden, E. Cheadle, S. D. Evans, J. R. Henderson, H. Fukushima, S. Miyashita, H. Schonherr, G. J. Vancso, R. Colorado, M. Graupe, O. E. Shmakova, T. R. Lee, Europhysics Letters 2002, 59, 410-416.

[107] S. D. Evans, H. Allinson, N. Boden, J. R. Henderson, Faraday Discussions 1996, 104, 37-48.

[108] A. M. Somoza, L. Mederos, D. E. Sullivan, Physical Review Letters 1994, 72, 3674-3677.

[109] D. K. Schwartz, Annual Review of Physical Chemistry 2001, 52, 107-137.

[110] E. Hecht, Optics, Addison-Wesley, 2002.

[111] Jasperso.Sn, Schnatte.Se, Review of Scientific Instruments 1969, 40, 761-\&. 
[112] H. G. Tomkins, E. A. Irene, Handbook of Ellipsometry, William Andrew Pub., 2005.

[113] A. W. Crook, Journal of the Optical Society of America 1948, 38, 954-964.

[114] A. Alessandrini, P. Facci, Measurement Science and Technology 2005, 16, R65.

[115] R. Lucht, Benetzung an der freien Oberfläche von thermotropen Flüssigkristallen, Görich \& Weiershäuser (Marburg), 2000.

[116] E. Kadivar, C. Bahr, H. Stark, Physical Review E 2007, 75.

[117] H. J. Coles, Molecular Crystals and Liquid Crystals 1978, 49, 67-74.

[118] R. J. Carlton, C. D. Ma, J. K. Gupta, N. L. Abbott, Langmuir 2012, 28, 12796-12805.

[119] R. J. Carlton, J. K. Gupta, C. L. Swift, N. L. Abbott, Langmuir 2011, 28, 31-36.

[120] U. Delabre, C. Richard, Y. Y. C. Sang, A.-M. Cazabat, Langmuir 2010, 26, 13368-13376.

[121] G. Akerlof, Journal of the American Chemical Society 1932, 54, 4125-4139.

[122] OPTIM ${ }^{\mathrm{TM}}$ Synthetic Glycerine - Refractive Index, The Dow Chemical Company http://www.dow.com/optim/optim-advantage/physical-properties/refractive.htm.

[123] M. Sjoeberg, U. Henriksson, T. Waernheim, Langmuir 1990, 6, 1205-1211.

[124] R. Palepu, H. Gharibi, D. M. Bloor, E. Wyn-Jones, Langmuir 1993, 9, 110-112.

[125] M. S. Bakshi, Journal of the Chemical Society, Faraday Transactions 1993, 89, 4323-4326.

[126] D. J. Lee, W. H. Huang, Colloid and Polymer Science 1996, 274, 160-165.

[127] C. C. Ruiz, J. A. Molina-Bolívar, J. Aguiar, G. MacIsaac, S. Moroze, R. Palepu, Langmuir 2001, 17, 6831-6840.

[128] C. Seguin, J. Eastoe, S. Rogers, M. Hollamby, R. M. Dalgliesh, Langmuir 2006, 22, 11187-11192.

[129] M. L. Moyá, A. Rodríguez, M. del Mar Graciani, G. Fernández, Journal of Colloid and Interface Science 2007, 316, 787-795.

[130] I. Langmuir, Journal of the American Chemical Society 1917, 39, 1848-1906.

[131] A. N. Frumkin, Zeitschrift für Physikalische Chemie 1925, 116, 466.

[132] J. Hopken, C. Pugh, W. Richtering, M. Moller, Makromolekulare Chemie-Macromolecular Chemistry and Physics 1988, 189, 911-925.

[133] N. Sakamoto, K. Sakai, K. Takagi, Journal of Chemical Physics 2000, 112, 946-953.

[134] R. Azzam, N. Bashara, S. S. Ballard, Ellipsometry and Polarized Light, North Holland, 1978.

[135] M. J. Uline, S. Meng, I. Szleifer, Soft Matter 2010, 6, 5482.

[136] Z. Zhang, H. Guo, Soft Matter 2012, 8, 5168-5174.

[137] Y. Iwashita, S. Herminghaus, R. Seemann, C. Bahr, Physical Review E 2010, 81, 051709.

[138] F. Dubreuil, J. Daillant, P. Guenoun, Langmuir 2003, 19, 8409-8415.

[139] L. M. Eng, C. Seuret, H. Looser, P. Gunter, Journal of Vacuum Science \& Technology B 1996, 14, 1386-1389.

[140] S. Herminghaus, A. Fery, D. Reim, Ultramicroscopy 1997, 69, 211-217.

[141] L. Bardin, M.-C. Faure, E. J. M. Filipe, P. Fontaine, M. Goldmann, Thin Solid Films 2010, 519, 414-416.

[142] A. Mourran, B. Tartsch, M. Gallyamov, S. Magonov, D. Lambreva, B. I. Ostrovskii, I. P. Dolbnya, W. H. de Jeu, M. Moeller, Langmuir 2005, 21, 2308-2316.

[143] G. Zhang, P. Marie, M. Maaloum, P. Muller, N. Benoit, M. P. Krafft, Journal of the American Chemical Society 2005, 127, 10412-10419.

[144] J. Daillant, G. Zalczer, J. J. Benattar, Physical Review A 1992, 46, R6158-R6161. 


\section{Acknowledgments}

First of all, I would like to thank Dr. Christian Bahr for his supervision on the whole process of my $\mathrm{PhD}$ research and the writing of this dissertation. I may say, without his instructions, advices and revisions, this $\mathrm{PhD}$ thesis would not have been finished. I also appreciate the freedom and space he has given to me. I will cherish for my whole life his understanding, support, and encouragement during my stay at the Max Planck Institute for Dynamics and SelfOrganization (MPIDS). I would like to thank Prof. Stephan Herminghaus for offering me the opportunity to work as a researcher at the institute. And his knowledge and attitude to science influence me and will guide me in my future academic life. I am indebted to Dr. Ahmed Mourran from RWTH, Aachen for his assistance and cooperation on the research done with AFM. Ahmed spent a lot of time on instructing me for the technique of AFM at RWTH, Aachen.

I have enjoyed a lot by working with all the members of the liquid crystal group at this institute. I will not forget the cooperation with Dr. Pramoda Kumar, Mr. Karthik Peddireddy, Mr. Benjamin Schulz, Dr. Anupam Sengupta, and Dr. Uros Tkalec.

I am very grateful to many people who help me during my PhD study at MPIDS. Special thanks from me should be given to the secretary Ms. Monika Teuteberg for her constant support in many aspects related to the administration of the institute. I take this opportunity to sincerely thank our IT technician Mr. Thomas Eggers. Thomas is always helpful not only in solving computer problems but also in my living in Göttingen. I would like to thank my two Chinese friends Mr. Patrick Tan and Mr. Song-Chuan Zhao of our institute for the funs they have shared with me.

I gratefully acknowledge Prof. Christoph Schmidt, Prof. Sarah Köster, and Prof. Marcus Müller from the University of Göttingen and Dr. Jean-Christophe Baret from MPIDS for their reviewing on this dissertation.

My acknowledgment should also be given to the European Union for offering me the Marie Curie fellowship and providing me with many opportunities to interact with other researchers within the network "Hierarchy".

Finally, I take this opportunity to thank my parents and my sister in China for their long-term encouragement. Many heartfelt thanks should go out to my girlfriend Ting for her love all these 
years, for being my companion in Europe, and for supporting me whenever I have difficulties. 


\section{Publications}

1. Xunda Feng, Ahmed Mourran, Martin Möller, and Christian Bahr AFM study of Gibbs films of semifluorinated alkanes at liquid crystal/air interfaces

ChemPhyChem 2013, accepted

2. Xunda Feng, Ahmed Mourran, Martin Möller, and Christian Bahr

Surface ordering and anchoring behaviour at liquid crystal surfaces laden with semifluorinated alkane molecules

Soft Matter 2012, 8 (37), 9661-9668

\section{Xunda Feng and Christian Bahr}

Surface order at surfactant-laden interfaces between isotropic liquid crystals and liquid phases with different polarity

Physical Review E 2011, 84(3), 031701(6 pages) 


\section{Curriculum Vitae}

\section{Xunda Feng}

Date of birth: 23-06-1983

Place of birth: Guangxi, China

Nationality: China

Personal address: Kellnerweg 12, Göttingen 37077, Germany

Education

09.2009 - present

PhD student in physics, with Prof. Stephan Herminghaus and Dr. Christian Bahr, Max Planck Institute for Dynamics and Self-Organization, Göttingen

$09.2006-07.2009$

M.E., applied chemistry, Department of Chemistry, Renmin University of China, Beijing, China

09.2002-07.2006

B.S., applied chemistry, Department of Applied Chemistry, Donghua University, Shanghai, China

\section{Research Experience}

2009-2012

2012 (2 months)

2010 (1 month)

2006-2009
EU Marie Curie researcher, with Prof. Stephan Herminghaus and Dr. Christian Bahr, Max Planck Institute for Dynamics and Self-Organization, Germany

Visiting PhD, with Prof. Martin Möller and Dr. Ahmed Mourran, Institute for Technical and Macromolecular Chemistry, RWTH Aachen, Germany

Visiting PhD, with Prof. Igor Musevic, the Jožef Stefan Institute, Slovenia

Research assistant, with Prof. Zhaoxia Jin, Renmin University of China

Göttingen, 05.03.2013 